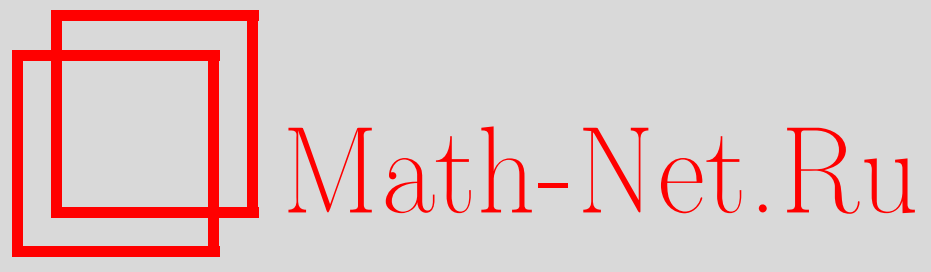

А. В. Пухликов, Бирационально жесткие многообразия. II. Расслоения Фано, УМН, 2010, том 65, выпуск 6, 87180

DOI: https://doi.org/10.4213/rm9368

Использование Общероссийского математического портала Math-Net.Ru подразумевает, что вы прочитали и согласны с пользовательским соглашением http://www . mathnet.ru/rus/agreement

Параметры загрузки:

IP: 35.173 .219 .12

26 апреля 2023 г., 16:37:06 


\title{
Бирационально жесткие многообразия. II. Расслоения Фано
}

\begin{abstract}
А. В. Пухликов
В статье дается обзор современного состояния теории бирациональной жесткости для расслоений Фано над базой положительной размерности. Описаны основные результаты, полученные в этой области за последние пятнадцать лет (бирациональная жесткость трехмерных многообразий с пучком поверхностей дель Пеццо, многомерных многообразий с пучком многообразий Фано и прямых произведений Фано), и техника метода максимальных особенностей для расслоений Фано. Статья непосредственно продолжает предыдущий обзор, посвященный бирациональной жесткости многообразий Фано.

Библиография: 54 названия.
\end{abstract}

Ключевые слова: многообразие Фано, расслоение Фано, бирациональная жесткость, послойная перестройка, максимальная особенность.

\section{СОДЕРЖАНИЕ}

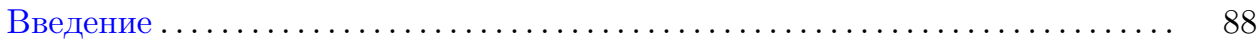

Глава 1. Бирациональная геометрия рационально связных расслоений ... 90

$\S 1$. Рационально связные расслоения ....................... 90

1.1. Расслоения Фано: определения и примеры................ 90

1.2. Послойные бирациональные отображения ................. 93

1.3. Замена структуры расслоения Фано .................. 97

$\S 2$. Программа минимальных моделей и программа Саркисова......... 99

2.1. Минимальные модели и расслоения Мори ................... 99

2.2. Проблема факторизации бирациональных отображений........ 103

2.3. О доказательстве программы Саркисова ................ 107

2.4. Метод максимальных особенностей и теория факторизации: сопоставление..................................... 111

$\S 3$. Бирациональная жесткость расслоений Фано ................... 115

3.1. Порог канонического присоединения.................... 115

3.2. Квадратичный метод: основные результаты .............. 118

3.3. Линейный метод: основные результаты ................ 123

(C) А. В. Пухликов, 2010 
Глава 2. Расслоения Фано над проективной прямой ................. 127

$\S 1$. Достаточные условия бирациональной жесткости ............... 127

1.1. Формулировка достаточных условий. Начало доказательства . . 127

1.2. Структура последовательности раздутий ............... 131

1.3. Кратности горизонтальных циклов .................... 133

1.4. Техника подсчета кратностей . . . . . . . . . . . . . . . . 135

1.5. Сверхмаксимальная особенность ................... 137

$\S 2$. Многообразия с пучком полных пересечений Фано............... 138

2.1. Расслоения на полные пересечения Фано .................. 138

2.2. Расслоения на циклические накрытия Фано ................ 140

2.3. Послойные бирациональные перестройки ............... 146

$\S 3$. Многообразия с пучком кубических поверхностей............... 148

3.1. Максимальные особенности. Существование прямой ... . . . . . . 148

3.2. Основная конструкция: лестница, связанная с прямой $L \ldots \ldots \ldots 150$

3.3. Техника подсчета кратностей ...................... 153

3.4. Цикл $Z^{(M)}$ в терминах лестницы .................... 154

3.5. Подсчет класса $z_{M}$ и завершение доказательства теоремы 1.7. . 154

Глава 3. Многообразия, имеющие много рационально связных структур . 156

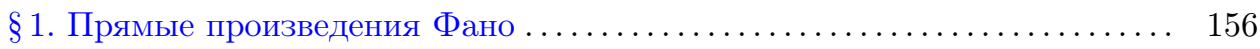

1.1. Максимальные особенности подвижных линейных систем...... 156

1.2. Редукция к базе расслоения........................ 159

1.3. Редукция к слою расслоения. Завершение доказательства бирациональной сверхжесткости многообразия $V \ldots \ldots \ldots \ldots \ldots \ldots .160$

1.4. Структуры рационально связного расслоения и бирациональные автоморфизмы.................................. 161

1.5. Пример многообразий, удовлетворяющих условию дивизориаль-

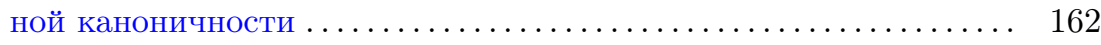

$\S 2$. Принцип связности и его приложения $\ldots \ldots \ldots \ldots \ldots \ldots \ldots \ldots \ldots \ldots \ldots \ldots$

2.1. Принцип связности. Обращение присоединения ............ 166

2.2. Дальнейшие приложения принципа связности .............. 168

2.3. Изолированные особенности . ....................... 170

2.4. Особенности пар на гладкой гиперповерхности ............. 171

2.5. Локальное неравенство для изолированной гиперповерхностной

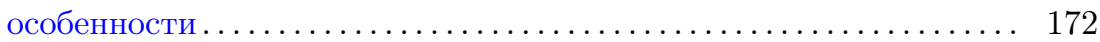

$\S 3$. Двойные пространства индекса два....................... 173

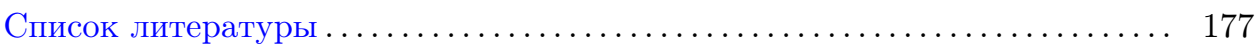

\section{Введение}

Цель данного обзора - описать полученные за последние пятнадцать лет результаты о бирациональной геометрии расслоений Фано над нетривиальной базой: трехмерных многообразий с пучком поверхностей дель Пеццо, многомерных многообразий с пучком многообразий Фано и прямых произведений Фано. 
Настоящая статья является непосредственным продолжением обзора [1], посвященного теории бирациональной жесткости многомерных многообразий Фано: рассматривается "относительный" вариант теории, т. е. изучаются расслоения Фано над базой положительной размерности. Если для типичных многообразий Фано феномен бирациональной жесткости заключался в отсутствии нетривиальных структур рационально связного расслоения, то для типичных расслоений Фано бирациональная жесткость означает единственность исходной структуры. Технической основой теории, как в абсолютном (многообразия Фано), так и в относительном (расслоения Фано) случае, является метод максимальных особенностей, однако работа с расслоениями над нетривиальной базой существенно сложнее во многих отношениях, требует дополнительных технических средств и потому теория расслоений Фано нуждается в отдельном (от абсолютного случая) рассмотрении. Геометрия многообразий Фано и расслоений Фано - это две ветви теории бирациональной жесткости, развивавшиеся параллельно. Именно техническими трудностями объясняется тот факт, что теорема Саркисова [2] вплоть до середины 1990-х годов оставалась единственным результатом о бирациональной жесткости расслоений Фано, и только статья [3] изменила положение, позволив успешно изучать новые классы расслоений.

Как и предыдущая статья [1], настоящий обзор предполагает знакомство читателя с основами алгебраической геометрии. Кроме того, предполагается знакомство с предыдущим обзором [1]. В частности, такие ключевые понятия, как рационально связное многообразие, порог канонического присоединения, максимальная особенность, бирациональная жесткость, бирациональная сверхжесткость и другие, предполагаются известными и используются без специальных пояснений. Это относится и к подробно изложенной в [1] технике гиперкасательных дивизоров.

Структура настоящего обзора аналогична [1]: первая глава менее формальна, ее цель - объяснить на примерах, какого вида многообразия являются предметом изучения, какие задачи рассматриваются и какие подходы имеются сегодня для их решения. Вторая глава посвящена бирациональной геометрии расслоений Фано над $\mathbb{P}^{1}$. В третьей главе обсуждаются многообразия, имеющие много нетривиальных структур рационально связного расслоения. В частности, кратко рассмотрены двойные пространства индекса два: это первый пример большого класса нежестких многообразий Фано, бирациональную геометрию которых удалось полностью описать методом максимальных особенностей. Отметим, что расслоениям на поверхности дель Пеццо степеней 1 и 2 посвящен недавний обзор [4] и поэтому этот класс многообразий нами не рассматривается.

Всюду далее термин "расслоение Фано" означает расслоение над базой положительной размерности, т. е. расслоение над нетривиальной базой.

Принцип нумерации утверждений, определений, замечаний и т. п. в работе тот же самый, что и в [1]: теорема (определение, лемма, замечание, . . ) $a . b$ есть теорема (определение, ... ) $b$ главы $a$; в каждой главе нумерация независима; при ссылке на параграф §a или пункт п. $a . b$, если номер главы не указан, то подразумевается текущая глава. 


\section{Глава 1}

\section{Бирациональная геометрия рационально связных расслоений}

\section{§ 1. Рационально связные расслоения}

1.1. Расслоения Фано: определения и примеры. Напомним, прежде всего, следующее общеизвестное определение.

ОПРЕДЕЛЕНИЕ 1.1. Сюръективный морфизм гладких проективных многообразий $\pi: V \rightarrow S$ со связными слоями одинаковой размерности называется расслоением Фано, если антиканонический класс $\left(-K_{V}\right)$ относительно обилен, т. е. обилен на слоях проекции $\pi$. Расслоение Фано называется стандартным, если

$$
\operatorname{Pic} V=\mathbb{Z} K_{V} \oplus \pi^{*} \operatorname{Pic} S,
$$

в частности, у стандартного расслоения Фано относительное число Пикара $\rho(V / S)$ равно 1 .

В настоящем обзоре рассматриваются только расслоения Фано над рационально связной базой $S$; в этом случае многообразие $V$ автоматически рационально связно. Если многообразие $V$ имеет $\mathbb{Q}$-факториальные терминальные особенности, $S$ нормально, а условие (1) заменено равенством $\rho(V / S)=1$, то такой более общий объект часто называют расслоением Mори, см. §2. Однако термин "расслоение Фано с особенностями" также является правомерным.

Слои расслоения Фано $\pi: V \rightarrow S$ являются многообразиями из некоторого семейства $\mathscr{F}$, общий элемент которого есть гладкое многообразие Фано, так что проекция $\pi$ порождает отображение $S \rightarrow \mathscr{F}$, сопоставляющее точке $s \in S$ соответствующий слой. В достаточно типичной ситуации пучок $\pi_{*}\left(-K_{V}\right)$ локально свободен и позволяет дать явную конструкцию расслоения Фано, как показывают следующие два примера.

Пример 1.1 (расслоения на полные пересечения Фано). Пусть $S$ - гладкое проективное рационально связное многообразие положительной размерности, $\mathscr{E}$ - локально свободный пучок на $S$ ранга $M+k+1$, где $M, k \geqslant 1$ - положительные целые числа, $X=\mathbb{P}(\mathscr{E})$ - соответствующее проективное расслоение в смысле Гротендика ( $X$ есть проективизация векторного расслоения, пучок сечений которого есть $\mathscr{E}^{*}-$ пучок, двойственный к $\left.\mathscr{E}\right)$. Проекция $\pi: X \rightarrow S$ есть локально тривиальное $\mathbb{P}^{M+k}$-расслоение. Если $L_{X} \in \mathrm{Pic} X-$ класс тавтологического пучка $\mathscr{O}_{\mathbb{P}(\mathscr{E})}(1)$, то

$$
\operatorname{Pic} X=\mathbb{Z} L_{X} \oplus \pi^{*} \operatorname{Pic} S,
$$

так что $X / S$ - расслоение Фано. Для канонического класса многообразия $X$ имеется хорошо известная формула

$$
K_{X}=-(M+k+1) L_{X}+\pi^{*}\left(\operatorname{det} \mathscr{E}+K_{S}\right) .
$$

Пусть $\left(d_{1}, \ldots, d_{k}\right) \in \mathbb{Z}_{+}^{k}-$ набор целых чисел, удовлетворяющих условиям

$$
d_{k} \geqslant \cdots \geqslant d_{1} \geqslant 2 \quad \text { и } \quad d_{1}+\cdots+d_{k}=M+k .
$$


Рассмотрим, далее, набор классов $A_{i} \in \operatorname{Pic} S, i=1, \ldots, k$, и предположим, что найдутся неприводимые дивизоры

$$
G_{i} \sim d_{i} L_{X}+\pi^{*} A_{i}, \quad i=1, \ldots, k,
$$

такие, что полное пересечение

$$
V=G_{1} \cap \cdots \cap G_{k} \subset X
$$

есть гладкое многообразие, слои $V \cap \pi^{-1}(s), s \in S$, которого имеют одинаковую размерность $M$. Ограничение проекции $\pi$ на $V$ обозначим тем же символом $\pi: V \rightarrow S$. Ограничение тавтологического класса $\left.L_{X}\right|_{V}$ обозначим символом $L_{V}$. Очевидно, что

$$
K_{V}=-L_{V}+\pi^{*} \Delta
$$

для некоторого $\Delta \in \operatorname{Pic} S$, так что $V / S$ - расслоение Фано. Более того, для точки общего положения $s \in S$ слой $\pi^{-1}(s) \subset V$ есть гладкое полное пересечение Фано типа $d_{1} \cdots d_{k}$ в $\mathbb{P}^{M+k}$. При $M \geqslant 3$ отсюда следует, что

$$
\operatorname{Pic} V=\mathbb{Z} L_{V} \oplus \pi^{*} \operatorname{Pic} S=\mathbb{Z} K_{V} \oplus \pi^{*} \operatorname{Pic} S,
$$

т. е. $V / S$ - стандартное расслоение Фано. Если $M=2$, то $V / S$ - расслоение на поверхности дель Пеццо степени 3 (здесь $k=1$ ), т. е. кубические поверхности в $\mathbb{P}^{3}$, или степени 4 (здесь $k=2, d_{1}=d_{2}=2$ ), т. е. полные пересечения типа $2 \cdot 2$ в $\mathbb{P}^{4}$. Если $M=1$, то $V / S$ есть расслоение на коники: в этом случае потребуем дополнительно, чтобы выполнялось неравенство $\operatorname{dim} S \geqslant 2$. При $M \in\{1,2\}$ условие (2) не будет выполняться автоматически и его нужно требовать дополнительно. Однако если дивизоры $G_{i}, 1 \leqslant i \leqslant k \in\{1,2\}$, обильны, то (2) выполнено по теореме Лефшеца. Приведенная конструкция дает наиболее "классический" пример стандартного расслоения Фано.

ПримеР 1.2 (расслоения на двойные накрытия Фано). Пусть $S, \mathscr{E}, X, \pi-$ такие же, как в предыдущем примере, $k=1$. Рассмотрим пару целых чисел $m \geqslant 2, l \geqslant 2$, удовлетворяющих равенству $m+l=M+1$. Предположим, что для некоторых классов $A_{Q}, A_{W} \in \operatorname{Pic} S$ имеются дивизоры

$$
Q \sim m L_{X}+\pi^{*} A_{Q} \quad \text { и } \quad W_{X} \sim 2\left(l L_{X}+\pi^{*} A_{W}\right),
$$

где $Q \subset X$ - гладкое подмногообразие, пересекающее каждый слой $\pi^{-1}(s)$, $s \in S$, по гиперповерхности (т. е. $Q$ не содержит целиком слоев проекции $\pi$ ), a $W_{X}$ высекает на $Q$ гладкий дивизор, не содержащий слоев проекции $\pi_{Q}=\left.\pi\right|_{Q}$. Этот набор объектов определяет двойное накрытие $\sigma: V \rightarrow Q$, разветвленное над дивизором $W=W_{X} \cap Q$. Слои проекции $\pi_{Q} \circ \sigma: V \rightarrow S$ суть $M$-мерные многообразия, причем общий слой есть гладкое примитивное многообразие Фано, откуда следует, что

$$
\operatorname{Pic} V=\mathbb{Z} K_{V} \oplus \sigma^{*} \pi_{Q}^{*} \operatorname{Pic} S,
$$

т. е. $V / S$ - стандартное расслоение Фано. Частный случай этой конструкции (соответствующий значению $m=1$ ) - это двойное накрытие $\sigma: V \rightarrow X$, разветвленное над гладким дивизором $W_{X} \sim 2\left((M+1) L_{X}+\pi^{*} A_{W}\right)$, не содержащим слоев проекции $\pi$ (дивизор $Q$ отсутствует); это - стандартное расслоение на двойные пространства Фано индекса единица. 
В каждом из рассмотренных примеров слои расслоения Фано $\pi: V \rightarrow S$ принадлежат к семейству $\mathscr{F} M$-мерных схем, общий элемент которого - гладкое многообразие Фано индекса один. Каждое из этих семейств допускает естественную структуру проективного многообразия, так что расслоению $V / S$ соответствует отображение $S \rightarrow \mathscr{F}$, сопоставляющее точке $s \in S$ слой $\pi^{-1}(s) \in \mathscr{F}$. В связи с этим возникает геометрическая характеристика “закрученности” расслоения $V / S$ как сложности отображения $S \rightarrow \mathscr{F}$. Например, если $V \subset \mathbb{P}^{1} \times \mathbb{P}^{3}$ гладкий дивизор бистепени $(d, 3)$, так что проекция $\pi: V \rightarrow \mathbb{P}^{1}$ на первый прямой сомножитель реализует $V$ как расслоение на поверхности дель Пеццо степени 3 , то закрученность этого расслоения по базе $\mathbb{P}^{1}$ легко определить как значение $d \geqslant 1$. Закрученность по базе есть интуитивно понятная степень сложности расслоения, и естественно ожидать, что чем выше закрученность, тем жестче структура расслоения. Приведем теперь пример наименее закрученного расслоения.

ПримеР 1.3 (прямые произведения Фано). Пусть $F_{1}, \ldots, F_{k}, k \geqslant 2,-$ примитивные многообразия Фано,

$$
V=F_{1} \times \cdots \times F_{k}
$$

- их прямое произведение, $\pi_{i}: V \rightarrow S_{i}=\prod_{j \neq i} F_{j}$ - проекция вдоль $F_{i}, i \in$ $\{1, \ldots, k\}$. В силу предположения, $\pi_{i}: V \rightarrow S_{i}$ есть стандартное расслоение Фано. Соответствующее отображение закрученности $S_{i} \rightarrow \mathscr{F}_{i}$ есть отображение в точку.

Напомним [1], что структурой рационально связного расслоения (или, короче, рационально связной структурой) на рационально связном многообразии $V$ называется бирациональное отображение $V \stackrel{\underset{\chi}{\rightarrow}}{\rightarrow} V^{+}$, где на многообразии $V^{+}$ зафиксирован морфизм $\pi^{+}: V^{+} \rightarrow S^{+}$, являющийся рационально связным расслоением. Расслоение Фано очевидным образом является рационально связным расслоением. Теперь основную проблему бирациональной геометрии расслоений Фано можно сформулировать так:

для заданного стандартного расслоения Фано $\pi: V \rightarrow S$ описать все структуры рачионально связного расслоения на многообразии $V$ по модулю отношения послойной бирациональной эквивалентности.

Говоря неформально, чем больше рационально связных структур имеет данное многообразие $V$, тем сложнее его бирациональная геометрия (в частности, наиболее сложную бирациональную геометрию имеет проективное пространство $\left.\mathbb{P}^{n}\right)$. Группа бирациональных автоморфизмов $\operatorname{Bir} V$ действует на множестве $R C(V)$ рационально связных структур, и наибольший интерес представляет фактормножество $\overline{R C}(V)$, введенное в [1]. Если $V / S$ - расслоение Фано над базой положительной размерности, то имеется важная подгруппа

$$
\operatorname{Bir}(V / S) \subset \operatorname{Bir} V
$$

послойных (относительно $\pi$ ) бирациональных автоморфизмов, снабженная естественным гомоморфизмом

$$
\operatorname{Bir}(V / S) \rightarrow \operatorname{Bir} S
$$


Ядро последнего гомоморфизма есть группа $\operatorname{Bir}\left(F_{\eta}\right)$ общего слоя (над общей точкой $S$ с полем вычетов $\mathbb{C}(S))$.

Первым этапом в решении поставленной выше общей проблемы естественно считать выявление таких расслоений Фано $V / S$, которые имеют в точности одну структуру рационально связного расслоения $\pi: V \rightarrow S$, либо, по крайней мере, эта структура единственна по модулю действия группы $\operatorname{Bir} V$. Такая единственность в большинстве случаев является следствием фундаментального свойства бирачиональной (сверх)жсесткости. Как отмечено в [1; гл. 1, п. 3.2], неформальный общий принцип состоит в том, что достаточно сильная закрученность по базе влечет бирациональную жесткость, единственность структуры $V / S$ и сводит бирациональную геометрию многообразия $V$ к бирациональной геометрии слоя $F_{\eta}$ над общей точкой. Реализация этого принципа будет рассмотрена ниже на различных примерах, полностью изученных к настоящему времени.

Метод максимальных особенностей, лежащий в основе доказательства почти всех результатов настоящего обзора, попутно решает и другие задачи: описывает группу $\operatorname{Bir} V$ и группу $\operatorname{Bir}(V / S)$. Современный вариант этого метода позволяет перейти к изучению многообразий с несколькими, и даже многими неэквивалентными структурами рационально связного расслоения. Многообразиям с единственной рационально связной структурой посвящена (главным образом) глава 2, многообразиям со многими структурами - глава 3.

В [1; гл. 1, 2] была объяснена и проиллюстрирована идея раскручивания бирационального отображения (или откручивания максимальных особенностей). По сути, это - универсальная идея упрощения сложного объекта путем последовательных элементарных шагов. Для многообразий Фано такими шагами обычно являются бирациональные инволюции [1; гл. $1, \S 3]$. Для расслоений Фано наиболее естественными кандидатами на роль упрощающих перестроек являются послойные перестройки: здесь, кроме автоморфизмов, возникают и операции другого типа. Неформальный принцип работы состоит в том, что бирациональное отображение $\chi: V \rightarrow V^{\prime}$ пространства расслоения Фано $V / S$ на пространство расслоения $V^{\prime} / S^{\prime}$ необходимо упрощать послойными перестройками до тех пор, пока либо оно не станет в некотором смысле "простым", либо на модифицированном пространстве $V$ не возникнет новая структура рационально связного расслоения, уже не согласованная с исходной структурой $V / S$. В последнем случае переход к новой структуре является упрощающим шагом.

Ниже рассмотрены примеры упрощающих модификаций обоих типов.

1.2. Послойные бирациональные отображения. В изучении бирациональной геометрии расслоения Фано $\pi: V \rightarrow S$ важную роль играют послойные бирациональные перестройки, т. е. коммутативные диаграммы отображений

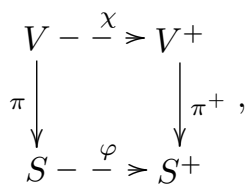


где $\chi$ и $\varphi$ - бирациональные отображения. Простейший тип таких перестроек дают бирациональные автоморфизмы общего слоя, $S^{+}=S, \varphi=\mathrm{id}, V^{+}=V$. Теперь, следуя [5], [3], рассмотрим пример.

ПримеР 1.4 (послойные бирациональные автоморфизмы пучка кубических поверхностей). В этом примере расслоение Фано есть частный случай конструкции примера 1.1. Пусть $\mathbb{P}(\mathscr{E}) \stackrel{\pi}{\rightarrow} \mathbb{P}^{1}-$ проективизация локально свободного пучка ранга 4 на $\mathbb{P}^{1}, V \subset \mathbb{P}(\mathscr{E})$ - гладкий дивизор, пересекающий каждый слой $\pi^{-1}(t)$ по кубической поверхности $F_{t} \subset \mathbb{P}^{3}=\pi^{-1}(t)$; предположим, что $V / \mathbb{P}^{1}$ стандартное расслоение Фано. Рассмотрим произвольное сечение $C \subset V$ проекции $\pi: V \rightarrow \mathbb{P}^{1}$. Для общей точки $t \in \mathbb{P}^{1}$ общая прямая $L \subset \mathbb{P}^{3}=G_{t}$, содержащая точку $C \cap F_{t}$, пересекает кубическую поверхность $F_{t}$ в еще двух различных точках $x, y$. Положим

$$
\tau_{C}(x)=y
$$

Очевидно, этим определена бирациональная инволюция $\tau_{C} \in \operatorname{Bir} F_{\eta} \subset \operatorname{Bir} V$. Пусть $\alpha: V^{*} \rightarrow V$ - раздутие кривой $C, E=\alpha^{-1}(C)$ - исключительный дивизор, Pic $V^{*}=\mathbb{Z} h \oplus \mathbb{Z} e \oplus \mathbb{Z} F$, где $h=-K_{V}$.

ЛЕмма 1.1. Бирационалъная инволюиия $\tau_{C}$ продолжается до бирегулярной инволюиии инвариантного открытого множества $V^{*} \backslash Y, \operatorname{codim} Y \geqslant 2$, и ее действие на $\operatorname{Pic} V^{*} / \mathbb{Z} F \cong \mathbb{Z} \bar{h} \oplus \mathbb{Z} \bar{e}$ дается соотношениями

$$
\tau_{C}^{*} \bar{h}=3 \bar{h}-4 \bar{e}, \quad \tau_{C}^{*} \bar{e}=2 \bar{h}-3 \bar{e} .
$$

ДокаЗАТЕЛЬСтво. См. [5].

Инволюции описанного выше типа традиционно называются инволюииями Гейзера.

Рассмотрим теперь произвольное бисечение $C \subset V$, т. е. неприводимую кривую, двулистно накрывающую базу $\mathbb{P}^{1}$. Определим инволюцию $\tau_{C}$ ее действием на общем слое $F$ следующим образом (см. [5], [3]). Пусть $\{a, b\}=C \cap F$ и $q=L_{a b} \cap F$ - третья точка пересечения прямой в $\mathbb{P}^{3}$, соединяющей точки $a$ и $b$, с кубической поверхностью $F$. Точки $q$ заметают кривую $C^{*} \subset V$ - сечение морфизма $\pi$, т. е. $q=C^{*} \cap F$. Пучок плоскостей $P$ в $\mathbb{P}^{3}$, содержащих прямую $L_{a b}$, порождает пучок эллиптических кривых $Q_{P}=P \cap F$ на поверхности $F$. Положим

$$
\left.\tau_{C}\right|_{Q_{P}}(x)=y,
$$

где $x+y \sim 2 q$ на $Q_{P}$, т. е. $\tau_{C}$ - отражение на эллиптической кривой $Q_{P}$ от точки $q$. Этим инволюция $\tau_{C} \in \operatorname{Bir} F_{\eta} \subset \operatorname{Bir} V$ определена.

Пусть снова $\alpha: V^{*} \rightarrow V$ - раздутие кривой $C, E=\alpha^{-1}(C)$ - исключительный дивизор, $\operatorname{Pic} V^{*}=\mathbb{Z} h \oplus \mathbb{Z} e \oplus \mathbb{Z} F$, где $h=-K_{V}$.

ЛЕмма 1.2. Бирациональная инволюиия $\tau_{C}$ продолжается до бирегулярной инволючии инвариантного открытого множества $V^{*} \backslash Y, \operatorname{codim} Y \geqslant 2$, и ее действие на $\operatorname{Pic} V^{*} / \mathbb{Z} F \cong \mathbb{Z} \bar{h} \oplus \mathbb{Z} \bar{e}$ дается соотношениями

$$
\tau_{C}^{*} \bar{h}=5 \bar{h}-6 \bar{e}, \quad \tau_{C}^{*} \bar{e}=4 \bar{h}-5 \bar{e} .
$$


ДокАЗАтЕльство. Непосредственные вычисления (см. [5]).

Инволюции описанного выше типа традиционно называются инволющиями Бертини.

Построенные бирациональные инволюции применяются для изучения геометрии многообразия $V$ следующим образом. Пусть $\Sigma \subset\left|-n K_{V}+l F\right|-$ подвижная линейная система на $V$, где $n \geqslant 1$, т. е. $\Sigma$ не составлена из пучка слоев. Кривая $C \subset V$ называется максимальной кривой системы $\Sigma$, если выполнено неравенство

$$
\operatorname{mult}_{C} \Sigma>n \text {. }
$$

Предположим, что $C$ - горизонтальная максимальная кривая, т. е. $\pi(C)=\mathbb{P}^{1}$ (иными словами, $C$ не содержится в слое $F_{t}$ ). Легко показать, что

$$
\left(K_{V}^{2} \cdot F\right)=3>\operatorname{deg}\left(\left.\pi\right|_{C}\right) \in\{1,2\},
$$

т. е. $C$ - сечение или бисечение проекции $\pi$. В силу лемм 1.1 и 1.2 легко убедиться, что собственный прообраз $\Sigma_{1}=\left(\tau_{C}\right)_{*} \Sigma$ линейной системы $\Sigma$ относительно инволюции $\tau_{C}$ удовлетворяет соотношению $\Sigma_{1} \subset\left|-n_{1} K_{V}+l_{1} F\right|$, где $n_{1}<n$. Точнее,

$$
n_{1}=3 n-2 \operatorname{mult}_{C} \Sigma
$$

если $C$ - сечение, и

$$
n_{1}=5 n-4 \text { mult }_{C} \Sigma,
$$

если $C$ - бисечение проекции $\pi$. Поскольку $n \in \mathbb{Z}_{+}$, через конечное число таких шагов приходим к системе, не имеющей максимальных кривых. Это - первый, более легкий, этап в изучении бирациональной геометрии многообразия $V$ и в доказательстве основной теоремы о бирациональной жесткости расслоения $V / \mathbb{P}^{1}[3]$, формулировка которой приведена в [1] (теорема 1.7). Второй, более трудный, этап (исключение бесконечно близких максимальных особенностей) обсуждается в $\S 3$ главы 2 настоящего обзора.

ПримеР 1.5 (послойные бирациональные перестройки расслоений на коники). Пусть $\pi: V \rightarrow S$ - стандартное расслоение на коники. Примеры сохраняющих слои бирациональных автоморфизмов приводились в [1; п. 2.3]. Однако для описания бирациональной геометрии многообразия $V$ (см. приведенную в [1; п. 2.3] теорему Саркисова и ее обсуждение) продуктивным оказывается не упрощение, как выше, линейной системы бирациональными автоморфизмами, а другой подход, который мы кратко опишем. Рассмотрим наиболее простую ситуацию: пусть слой $C=\pi^{-1}(p)$ над точкой $p \in S$ - неособая коника, $C \cong \mathbb{P}^{1}$. Раздуем одновременно точку $p$ на поверхности $S$ и кривую $C$ на многообразии $V$ :

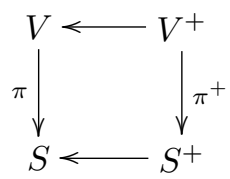

(проекцию $V^{+} \rightarrow S^{+}$для простоты обозначаем тем же символом $\pi$ ). Пусть $E \subset V^{+}$и $E_{S} \subset S^{+}$- исключительные дивизоры этих раздутий. Отметим, что 
$V^{+} / S^{+}$- снова стандартное расслоение на коники. Очевидно, что $E=E_{S} \times \mathbb{P}^{1}$. Пусть $\Sigma \subset\left|-n K_{V}+\pi^{*} A\right|-$ подвижная линейная система, $\mu=\operatorname{mult}_{C} \Sigma$ и

$$
\Sigma^{+} \subset\left|-n K_{V^{+}}+\pi^{*}\left(A+(n-\mu) E_{S}\right)\right|
$$

- ее собственный прообраз на $V^{+}$. Слой $C$ является максимальной кривой системы $\Sigma$, если $\mu>n$, и этот пример показывает, как устранить все максимальные кривые этого типа, модифицируя расслоение на коники (если $C$ - компонента приводимого слоя или носитель неприведенного слоя, то конструкция бирациональной перестройки гораздо сложнее, см. [2]). Предположим теперь, что некоторая кривая $\Gamma \subset E$, не являющаяся слоем $\pi$, удовлетворяет условию

$$
\nu+\mu>2 n
$$

где $\nu=$ mult $_{\Gamma} \Sigma^{+}>n$, т. е. является бесконечно близкой максимальной кривой исходной системы $\Sigma$. Поскольку $\Sigma^{+}$не имеет неподвижных компонент и для общего дивизора $D^{+} \in \Sigma^{+}$имеем

$$
\left(D^{+} \cdot \pi^{-1}(s)\right)=-n\left(K_{V^{+}} \cdot \pi^{-1}(s)\right)=2 n,
$$

кривая Г является сечением линейчатой поверхности $E / E_{S}$, в частности, Г есть гладкая рациональная кривая. Пусть $\varphi: V^{\sharp} \rightarrow V^{+}-$бирациональная перестройка, являющаяся композицией двух операций: раздутия кривой $Г$ и последующего послойного стягивания собственного прообраза линейчатой поверхности $E$. Получаем новое стандартное расслоение на коники $\pi: V^{\sharp} \rightarrow S^{+}$, для собственного прообраза линейной системы $\Sigma$ на $V^{\sharp}$ имеем

$$
\Sigma^{\sharp} \subset\left|-n K_{V^{\sharp}}+\pi^{*}\left(A+(3 n-2 \mu-\nu) E_{S}\right)\right| .
$$

Отметим еще раз, что если максимальная особенность системы $\Sigma$ лежит над точкой особого или неприведенного слоя проекции $\pi$, то необходимая перестройка существенно сложнее описанной конструкции. Однако сказанное уже позволяет объяснить идею доказательства теоремы Саркисова [2] (в [1] это теорема 1.6). Пусть

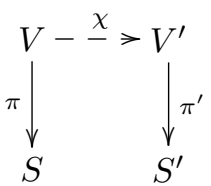

- бирациональное отображение между двумя расслоениями на коники, где $V$ удовлетворяет условию $\left|4 K_{S}+\Delta\right| \neq \varnothing$, а $\Delta \subset S$ - дискриминантный дивизор. Поднимая с базы $S^{\prime}$ очень обильную линейную систему, получим подвижную систему $\Sigma^{\prime}$ на $V^{\prime}$. Пусть $\Sigma \subset\left|-n K_{V}+\pi^{*} A\right|$ - ее собственный прообраз на $V$. Если $\chi$ не является послойным, то $n \geqslant 1$. Предположим поэтому, что $n \geqslant 1$. Нетрудно проверить, что условие $\left|4 K_{S}+\Delta\right| \neq \varnothing$ инвариантно относительно послойных перестроек (в приведенном выше примере это очевидно). Поэтому, применяя послойные перестройки, можно считать, что линейная система $\Sigma$ не имеет максимальных особенностей. Из условия обрыва присоединения легко 
вывести, что в этом случае класс $A \in \operatorname{Pic} S$ не эффективен (точнее, не псевдоэффективен). Вычислим теперь самопересечение

$$
Z=\left(D_{1} \circ D_{2}\right), \quad D_{i} \in \Sigma,
$$

линейной системы $\Sigma$ и опустим его на $S$ :

$$
\pi_{*} Z \sim n^{2} \pi_{*} K_{V}^{2}+4 n A \sim-n^{2}\left(4 K_{S}+\Delta\right)+4 n A .
$$

Отсюда в силу предположения следует, что

$$
A \sim \frac{n}{4}\left(4 K_{S}+\Delta\right)+\frac{1}{4 n} \pi_{*} Z
$$

- эффективный класс. Полученное противоречие доказывает теорему Саркисова. Детали см. в [2].

1.3. Замена структуры расслоения Фано. В классе расслоений Фано со слоем размерности три и выше послойные перестройки встречаются гораздо реже. Следующий пример показывает, насколько более жесткой является послойная бирациональная геометрия в высших размерностях.

ПримеР 1.6 (отсутствие нетривиальных послойных бирациональных соответствий в высших размерностях [6]). Пусть $C$ - гладкая аффинная кривая с отмеченной точкой $p \in C$, и $C^{*}=C \backslash\{p\}-$ проколотая кривая. Пусть $\mathscr{V}(d)$, $d \geqslant 2,-$ множество гладких дивизоров $V \subset X=C \times \mathbb{P}^{M}, M \geqslant 3$, каждый слой которых

$$
F_{x}=V \cap\{x\} \times \mathbb{P}^{M}, \quad x \in C
$$

- гиперповерхность степени $d$. Положим

$$
X^{*}=C^{*} \times \mathbb{P}^{M}, \quad V^{*}=V \cap X^{*},
$$

так что $V^{*}$ получается из $V$ выбрасыванием слоя $F_{p}$ над отмеченной точкой. Предположим, что $d \geqslant 3$ и $V_{1}, V_{2} \in \mathscr{V}(d)$. Имеет место следующий факт [6].

ТеОрема 1.1. Пусть $\chi^{*}: V_{1}^{*} \rightarrow V_{2}^{*}-$ послойный изоморфизм. Тогда $\chi^{*}$ продолжается до послойного изоморбизма $\chi: V_{1} \rightarrow V_{2}$.

Доказательство будет дано в п. 2.3

Следующий пример посвящен многообразиям с двумя структурами расслоений Фано. Переход от одной структуры к другой является искомой упрощающей перестройкой. Другие аналогичные примеры см. в [7], [8].

ПримеР 1.7. Рассмотрим следующий частный случай конструкции примеpa 1.1: пусть $\mathscr{E}=\mathscr{O}_{\mathbb{P}^{1}}^{\oplus(M-1)} \oplus \mathscr{O}_{\mathbb{P}^{1}}(1)^{\oplus 2}-$ локально свободный пучок ранга $(M+1)$ на $\mathbb{P}^{1}, X=\mathbb{P}(\mathscr{E})$ - соответствующее $\mathbb{P}^{M}$-расслоение над $\mathbb{P}^{1}, \operatorname{Pic} X=\mathbb{Z} L_{X} \oplus \mathbb{Z} R$, где $R$ - класс слоя проекции $\pi_{X}: X \rightarrow \mathbb{P}^{1}, L_{X}$ - тавтологический класс. Пусть $V \sim M L_{X}$ - общий дивизор. Это - гладкое многообразие, расслоенное проекцией $\pi=\left.\pi_{X}\right|_{V}: V \rightarrow \mathbb{P}^{1}$ на гиперповерхности Фано индекса один. Очевидно, что $V / \mathbb{P}^{1}$ - стандартное расслоение Фано, Pic $V=\mathbb{Z} K_{V} \oplus \mathbb{Z} F$, где $F$ - класс 
слоя проекции $\pi$ и $K_{V}=-L, L=\left.L_{X}\right|_{V}$. На многообразии $V$ имеется, однако, и другая структура рационально связного расслоения.

Рассмотрим локально свободные подпучки

$$
\mathscr{E}_{0}=\mathscr{O}_{\mathbb{P}^{1}}^{\oplus(M-1)} \hookrightarrow \mathscr{E} \quad \text { и } \quad \mathscr{E}_{1}=\mathscr{O}_{\mathbb{P}^{1}}(1)^{\oplus 2} \hookrightarrow \mathscr{E} .
$$

Очевидно, что $\mathscr{E}=\mathscr{E}_{0} \oplus \mathscr{E} 1$. Пусть $\mathscr{L}_{X}$ - тавтологический пучок Гротендика расслоения $\mathbb{P}(\mathscr{E})$ и $\Pi_{0} \subset H^{0}\left(X, \mathscr{L}_{X}\right)$ - подпространство, соответствующее пространству сечений подпучка $H^{0}\left(\mathbb{P}^{1}, \mathscr{E}_{0}\right) \hookrightarrow H^{0}\left(\mathbb{P}^{1}, \mathscr{E}\right)$. Положим также

$$
\Pi_{1}=H^{0}\left(X, \mathscr{L}_{X} \otimes \pi^{*} \mathscr{O}_{\mathbb{P}^{1}}(-1)\right)=H^{0}\left(\mathbb{P}^{1}, \mathscr{E}_{1}(-1)\right)
$$

Пусть $x_{0}, \ldots, x_{M-2}$ - некоторый базис пространства $\Pi_{0}$, а $y_{0}, y_{1}$ - базис пространства $\Pi_{1}$. Тогда сечения

$$
x_{0}, \ldots, x_{M-2}, y_{0} t_{0}, y_{0} t_{1}, y_{1} t_{0}, y_{1} t_{1},
$$

где $t_{0}, t_{1}$ - система однородных координат на $\mathbb{P}^{1}$, образуют базис пространства $H^{0}\left(X, \mathscr{L}_{X}\right)$. Легко видеть, что эта полная линейная система задает морфизм

$$
\xi: X \rightarrow \bar{X} \subset \mathbb{P}^{M+2},
$$

образ $\bar{X}$ которого есть квадратичный конус с вершинным пространством $\mathbb{P}^{M-2}=\xi\left(\Delta_{X}\right)$ и базой - неособой квадрикой в $\mathbb{P}^{3}$, изоморфной $\mathbb{P}^{1} \times \mathbb{P}^{1}$, где $\Delta_{X}=\mathbb{P}\left(\mathscr{E}_{0}\right)$ есть базисное множество пучка $\left|L_{X}-R\right|, \Delta_{X}=\mathbb{P}^{M-2} \times \mathbb{P}^{1}$, стягиваемое отображением $\xi$ на первый сомножитель. Морфизм $\xi$ бирационален, точнее,

$$
\xi: X \backslash \Delta_{X} \rightarrow \bar{X} \backslash \xi\left(\Delta_{X}\right)
$$

есть изоморфизм, причем $\xi$ стягивает $\Delta_{X}=\mathbb{P}^{M-2} \times \mathbb{P}^{1}$ на вершинное пространство конуса. Пусть

$$
u_{0}, \ldots, u_{M-2}, u_{00}, u_{01}, u_{10}, u_{11}
$$

- однородные координаты на $\mathbb{P}^{M+2}$, соответствующие упорядоченному набору сечений (3). Конус $\bar{X}$ задается уравнением

$$
u_{00} u_{11}=u_{01} u_{10}
$$

На конусе $\bar{X}$ имеются два пучка $M$-плоскостей, соответствующих двум пучкам прямых на гладкой квадрике в $\mathbb{P}^{3}$. Пусть $\tau \in$ Aut $\mathbb{P}^{M+2}$ - автоморфизм, переставляющий координаты $u_{01}$ и $u_{10}$ и не меняющий других координат. Очевидно, что $\tau \in \operatorname{Aut} \bar{X}$ есть автоморфизм конуса $\bar{X}$, переставляющий вышеупомянутые пучки $M$-плоскостей. Один из этих пучков есть образ пучка слоев проекции $\pi$, т. е. пучок $\xi(|R|)$. Для другого пучка имеем очевидное равенство

$$
\tau \xi(|R|)=\xi\left(\left|L_{X}-R\right|\right) .
$$

Автоморфизм $\tau$ индуцирует инволютивный бирациональный автоморфизм

$$
\tau^{+} \in \operatorname{Bir} X
$$


Точнее, $\tau^{+}$есть бирегулярный автоморфизм вне замкнутого подмножества $\Delta_{X}$ коразмерности 2 . Пусть $\varepsilon: \widetilde{X} \rightarrow X$ - раздутие гладкого подмногообразия $\Delta_{X}$. Очевидно, что многообразие $\tilde{X}$ изоморфно раздутию конуса $\bar{X}$ в его вершинном пространстве $\xi\left(\Delta_{X}\right)$. Легко проверить, что $\tau^{+}$продолжается до бирегулярного автоморфизма гладкого многообразия $\widetilde{X}$. Линейные системы $\left|k L_{X}\right|$, $k \in \mathbb{Z}_{+}$, инвариантны относительно $\tau^{+}$. В частности, для общего дивизора $V \in\left|M L_{X}\right|$ его $\tau^{+}$-образ $V^{+}=\tau^{+}(V)$ - общий дивизор той же линейной системы, в частности, $V^{+}$- гладкое многообразие. Отметим, что если $V \in\left|M L_{X}\right|$ задан уравнением

$$
h\left(u_{0}, \ldots, u_{M-2}, u_{00}, u_{01}, u_{10}, u_{11}\right)=0,
$$

то его образ $V^{+}$задается уравнением

$$
h^{+}\left(u_{*}\right)=h\left(u_{0}, \ldots, u_{M-2}, u_{00}, u_{10}, u_{01}, u_{11}\right)=0,
$$

где переставлены координаты $u_{01}$ и $u_{10}$.

Таким образом, получаем два расслоения Фано, $V / \mathbb{P}^{1}$ и $V^{+} / \mathbb{P}^{1}$, связанные бирациональным изоморфизмом $\tau^{+}: V \rightarrow V^{+}$, который не является послойным. Это бирациональное отображение бирегулярно в коразмерности единица и действует на группе Пикара следующим образом:

$$
\left(\tau^{+}\right)^{*} K_{V^{+}}=K_{V}, \quad\left(\tau^{+}\right)^{*} F^{+}=-K_{V}-F,
$$

где $F^{+}-$класс слоя проекции $V^{+} \rightarrow \mathbb{P}^{1}$, так что $\mathrm{Pic} V^{+}=\mathbb{Z} K_{V^{+}} \oplus \mathbb{Z} F^{+}$. По построению конструкция инволютивна, т. е. $\left(V^{+}\right)^{+}=V$.

Отметим, что бирациональное отображение $\tau^{+}: V \rightarrow V^{+}$есть композиция раздутия подмногообразия $\Delta=\Delta_{X} \cap V$ коразмерности два и последующего стягивания исключительного дивизора на подмногообразие $\Delta^{+}=\Delta_{X} \cap V^{+}$.

Переход с модели $V$ на модель $V^{+}$посредством бирационального отображения $\tau^{+}$используется как упрощающая перестройка следующим образом. Пусть

$$
\Sigma \subset\left|-n K_{V}+l F\right|
$$

- подвижная система. Если $l<0$, то линейная система $\tau_{*}^{+} \Sigma \subset\left|-n_{+} K_{V^{+}}+l_{+} F^{+}\right|$ имеет параметры

$$
n_{+}=n+l, \quad l_{+}=-l \geqslant 1 .
$$

\section{§ 2. Программа минимальных моделей и программа Саркисова}

2.1. Минимальные модели и расслоения Мори. Программа минимальных моделей (ПММ) обобщает в высшие $(\geqslant 3)$ размерности классическую теорию минимальных моделей алгебраических поверхностей [9]. Смысл ПММ состоит в том, чтобы путем явно описываемых бирациональных перестроек сопоставить каждому алгебраическому многообразию некоторую "модель", обладающую "хорошими свойствами" в отношении канонического класса. Если для гладкой проективной поверхности достаточно стягивать исключительные прямые (или (-1)-кривые), чтобы прийти либо к минимальной поверхности 
с численно эффективным каноническим классом, либо к линейчатой поверхности (т. е. $\mathbb{P}^{1}$-расслоению над гладкой проективной кривой), то в размерности три и выше ситуация намного сложнее:

- экстремальные стягивания (многомерные аналоги операции стягивания $(-1)$-кривой) неизбежно приводят к многообразиям с особенностями, даже если исходное многообразие неособо;

- возникает новый тип бирациональных перестроек, являющихся изоморфизмами в коразмерности 1, т. е. вне некоторого замкнутого подмножества коразмерности $\geqslant 2$; именно эти перестройки и порождают наибольшие технические трудности;

- как стало ясно начиная с середины 80-х годов, технически более естественным объектом является не алгебраическое многообразие $X$, а пара (или логnара) $(X, \Delta)$, где $\Delta$ - некоторая граница, представляющая собой (как правило, эффективный $\mathbb{Q}$ - (или $\mathbb{R}-)$ дивизор Вейля на $X$ такой, что $K_{X}+\Delta$ есть $\mathbb{Q}$ - (соответственно, $\mathbb{R}-$-) дивизор Картье. Работа с парами позволяет охватывать любые типы особенностей и хорошо приспособлена для построения индуктивных процедур ПММ.

Пусть $X$ - нормальное проективное многообразие, $\Delta$ - эффективный $\mathbb{R}$-дивизор Вейля, $\Delta=\sum d_{i} \Delta_{i}$, где $\Delta_{i} \subset X$ - различные простые дивизоры. Пусть $f: Y \rightarrow X-$ логразрешение пары $(X, \Delta)$, т. е. такая последовательность раздутий, что $Y$ - гладкое проективное многообразие и

$$
\bigcup \Delta_{i}^{+} \cup \bigcup E_{j}
$$

есть дивизор с нормальными пересечениями, где $\bigcup E_{j}-$ исключительный дивизор морфизма $f, \Delta_{i}^{+}-$собственный прообраз дивизора $\Delta_{i}$ на $Y$. Записывая

$$
K_{Y}+\Delta^{+}=f^{*}\left(K_{X}+\Delta\right)+\sum a_{j} E_{j}
$$

(где $\Delta^{+}=\sum d_{i} \Delta_{i}^{+}$; напомним, что $K_{X}+\Delta$ есть $\mathbb{R}$-Картье дивизор), скажем, что пара $(X, \Delta)$ есть klt-napa (Кавамата логтерминальна), если все $d_{i}<1$ и все $a_{j}>-1$.

Имеется два наиболее важных вида klt-пар:

1) минимальные модели (или логтерминальные модели), когда $K_{X}+\Delta$ численно эффективен,

2) расслоения Мори, когда имеется морфизм $\varphi: X \rightarrow S$ на нормальное проективное многообразие $S$, причем $\rho(X / S)=1, \varphi_{*} \mathscr{O}_{X}=\mathscr{O}_{S}$ и $-\left(K_{X}+\Delta\right)$ обилен относительно $\varphi$.

Цель ПММ - путем бирациональных перестроек специального вида получить из произвольной klt-пары $(X, \Delta)$ либо минимальную модель, либо расслоение Мори:

$$
X_{0}=X \stackrel{f_{1}}{-} \rightarrow X_{1}-\stackrel{f_{2}}{-} \rightarrow \cdots-\stackrel{f_{N}}{-} \rightarrow X_{N},
$$

$\Delta_{0}=\Delta, \Delta_{i}=\left(f_{i}\right)_{*} \Delta_{i-1}$ и $\left(X_{N}, \Delta_{N}\right)$ имеет тип 1) или 2). Каждое бирациональное отображение $f_{i+1}: X_{i} \rightarrow X_{i+1}$ есть либо $\left(K_{X_{i}}+\Delta_{i}\right)$-экстремальное дивизориальное стягивание, либо флип относительно малого $\left(K_{X_{i}}+\Delta_{i}\right)$-экстремального стягивания $\varphi_{i}: X_{i} \rightarrow Y_{i}$, т. е. рациональное отображение $\varphi_{i}^{+}=$ $\varphi_{i} \circ f_{i+1}^{-1}: X_{i+1} \rightarrow Y_{i}$ есть малый бирациональный морфизм и $K_{X_{i+1}}+\Delta_{i+1}$ 
относительно $\varphi_{i}^{+}$-обилен. Существование флипов в произвольной размерности и наибольшей общности было доказано в [10]. Основной трудностью в построении ПММ с самого начала была проблема конечности, т. е. обрыва последовательности флипов. В самом деле, последовательность дивизориальных стягиваний по очевидным причинам не может быть бесконечной. Поэтому алгоритм ПММ приводит к желаемому результату (минимальной модели или расслоению Мори) при условии, что последовательность перестроек флипового типа не может быть бесконечной. В [10] существование минимальных моделей было доказано путем модификации основного подхода: вместо того, чтобы обосновывать конечность произвольной последовательности флипов, достаточно построить (или доказать существование) некоторой последовательности, обрывающейся через конечное число шагов. Это и было сделано в [10]. Приведем основной результат этой работы.

Tеорема 1.2 [10]. Пусть $(X, \Delta)$ - klt-napa, где $K_{X}+\Delta$ ecmъ $\mathbb{R}$-Kapmъе $\partial u$ визор, $и \pi: X \rightarrow U-$ проективный морфизм квазипроективных многообразий. Предположим, что либо $\Delta$ является $\pi$-обгемным, $а K_{X}+\Delta-\pi$-псевдоэфбективным, либо $K_{X}+\Delta$ является $\pi$-обгемным. Тогда:

1) пара $(X, \Delta)$ имеет логтерминальную модель над $U$;

2) если $K_{X}+\Delta$ является $\pi$-обгемным, то $(X, \Delta)$ имеет логканоническую модель над $U$;

3) если $K_{X}+\Delta$ есть $\mathbb{Q}$-Картъе дивизор, то $\mathscr{O}_{U}$-алгебра

$$
\bigoplus_{m \in \mathbb{N}} \pi_{*} \mathscr{O}_{X}\left(\left\llcorner m\left(K_{X}+\Delta\right)\right\lrcorner\right)
$$

конечно порождена.

Этот весьма технический результат влечет за собой ряд важных геометрических следствий. Мы приведем три утверждения, из которых для настоящей работы наиболее важно последнее.

СЛЕДСТВИЕ 1.1 [10]. Пусть $X-$ гладкое проективное многообразие общего типа. Тогда $X$ имеет минимальную модель, каноническое кольцо

$$
\bigoplus_{m \in \mathbb{N}} H^{0}\left(X, \mathscr{O}_{X}\left(m K_{X}\right)\right)
$$

конечно порождено и X имеет модель, обладающую метрикой Кэлера-Эйнштейна.

СлеДСтвие 1.2 [10]. Пусть $(X, \Delta)$ - klt-napa $u \pi: X \rightarrow Z-$ малое $\left(K_{X}+\Delta\right)$ экстремальное стягивание. Тогда для $\pi$ существует флип.

СлеДСТвиЕ 1.3 [10]. Пусть $(X, \Delta)$ есть klt-nара, причем $X-\mathbb{Q}$-факториальное многообразие. Пусть $\pi: X \rightarrow U-$ проективный морфизм нормальных квазипроективных многообразий, причем $K_{X}+\Delta$ не является $\pi$-псевдоэфбективным. Тогда некоторая последовательность ПММ-перестроек

$$
f=f_{N} \circ \cdots \circ f_{1}: X \rightarrow Y
$$

приводит к расслоению Мори $g: Y \rightarrow W$ над $U$. 
Из последнего утверждения немедленно вытекает, что любое рационально связное многообразие $X$ бирационально эквивалентно расслоению Мори:

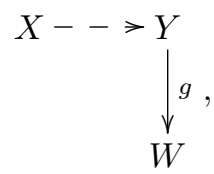

где $Y$ - многообразие с $\mathbb{Q}$-факториальными терминальными особенностями, $W$ - проективное нормальное многообразие и $\left(-K_{Y}\right)$ обилен относительно $g$. Аналогичным образом, для любого рационально связного расслоения $\pi: X \rightarrow S$ имеется коммутативная диаграмма

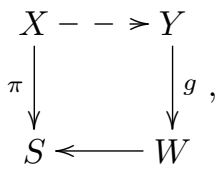

где верхняя стрелка - бирациональное отображение и $Y / W$ - расслоение Мори. (Отметим, что, поскольку обрыв произвольной последовательности флипов до сих пор является открытой проблемой, следствие 1.3 не утверждает, что любая последовательность ПММ-перестроек приводит к расслоению Мори.)

Из сказанного следует, что все задачи бирациональной геометрии рационально связных многообразий могут быть ограничены категорией расслоений Мори: вместо того, чтобы выяснять, существует ли бирациональная эквивалентность $X_{1} \rightarrow X_{2}$ между двумя рационально связными многообразиями, можно заменить $X_{i}$ бирационально эквивалентным расслоением Мори $\pi_{i}: Y_{i} \rightarrow S_{i}$ и рассмотреть эту задачу для $Y_{1}, Y_{2}$. Если $X_{1}, X_{2}$ бирационально эквивалентны, то описание всех бирациональных отображений $X_{1} \rightarrow X_{2}$ эквивалентно описанию группы бирациональных автоморфизмов $\operatorname{Bir} X_{i}=\operatorname{Bir} Y_{i}$. Если $\pi: X \rightarrow R-$ рационально связное расслоение, то проблема описания соотношения между группой $\operatorname{Bir} X$ и группой послойных бирациональных автоморфизмов $\operatorname{Bir}(X / R)$ также переносится на соответствующее расслоение Мори $Y / S$.

Ограничение задач бирациональной геометрии рамками категории расслоений Мори имеет ряд несомненных преимуществ. Например, устраняется асимметрия традиционного подхода, когда изучались бирациональные отображения $\chi: V \rightarrow V^{\prime}$, где многообразие $V$ принадлежало к категории расслоений Фано, а $V^{\prime}-$ к категории рационально связных многообразий (или расслоений); если же работать с расслоениями Мори (т. е. с расслоениями Фано с $\mathbb{Q}$-факториальными терминальными особенностями и относительным числом Пикара, равным единице), то оба многообразия принадлежат к одной и той же категории. Далее, мотивированный ПММ-подход позволяет поставить и решить общую задачу факторизации бирациональных отображений в композицию элементарных перестроек (линков), которую мы рассмотрим в следующем пункте.

И тем не менее, неясно, насколько эти преимущества существенны и оправдывают ли они отказ от традиционного подхода, насколько “лучше" или "хуже" основные определения (например, ключевого понятия бирациональной жесткости), соответствующие идеологии ПММ; этот вопрос будет обсуждаться ниже. 
2.2. Проблема факторизации бирациональных отображений. Программа Саркисова - это теория факторизации бирациональных отображений между расслоениями Мори в композицию элементарных перестроек (линков). Рассмотрим диаграмму

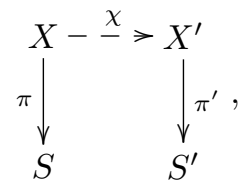

где $\chi$ - бирациональное отображение, $X / S$ и $X^{\prime} / S^{\prime}$ - расслоения Мори. Необходимо построить последовательность промежуточных расслоений Мори $\pi_{i}$ : $X_{i} \rightarrow S_{i}, i=0,1, \ldots, N$, начинающуюся с $\pi_{0}=\pi\left(X_{0}=X\right.$ и $\left.S_{0}=S\right)$ и кончающуюся $\pi_{N}=\pi^{\prime}\left(X_{N}=X^{\prime}\right.$ и $\left.S_{N}=S^{\prime}\right)$, и последовательность элементарных перестроек (линков) $\tau_{i}: X_{i-1} \rightarrow X_{i}$ такие, что

$$
\chi=\tau_{N} \circ \cdots \circ \tau_{1}: X_{0}=X \rightarrow X_{N}=X^{\prime} .
$$

Напомним, что линк $\tau: X \rightarrow Y$ между расслоениями Мори $\pi: X \rightarrow S$ и $\rho: Y \rightarrow T-$ это бирациональное отображение одного из следующих четырех типов.

Тип I (расширение базы). Имеются: экстремальное дивизориальное стягивание $\varphi: Z \rightarrow X$, бирациональное отображение $\psi: Z \rightarrow Y$, являющееся композицией флопов (в частности, изоморфизм в коразмерности единица) и экстремальное стягивание $\varepsilon: T \rightarrow S$ (в частности, $\rho(T / S)=1$ ) такие, что коммутативна диаграмма отображений

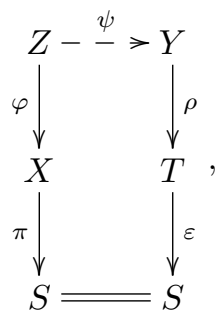

причем $\psi \circ \varphi^{-1}=\tau: X \rightarrow Y$. Простейший пример линка этого типа мы видели выше (пример 1.5), он соответствовал раздутию точки $\varepsilon: T \rightarrow S$ на базе расслоения на коники и раздутию $\varphi: Z \rightarrow X$ слоя над этой точкой. Приведем еще один пример.

ПримеР 1.8. Пусть $L \subset \mathbb{P}^{3}$ - прямая, $\sigma: X \rightarrow \mathbb{P}^{3}$ - ее раздутие, $\Pi_{L}-$ пучок плоскостей в $\mathbb{P}^{3}$, содержащих прямую $L$, а $\Pi_{L}^{+}$- его собственный прообраз на $X$. Очевидно, что $\Pi_{L}^{+}$есть пучок слоев морфизма

$$
\pi: X \rightarrow S=\mathbb{P}^{1}
$$

со слоем $\mathbb{P}^{2}$, т. е. $X / S$ есть расслоение Мори (и расслоение Фано в традиционном смысле). Пусть теперь $R \subset X-$ произвольное сечение проекции $\pi$ и $\varphi: Z \rightarrow X-$ ее раздутие. Сквозное отображение

$$
\pi \circ \varphi: Z \rightarrow \mathbb{P}^{1}
$$


есть расслоение на рациональные линейчатые поверхности типа $\mathbb{F}_{1}$. Точнее, пусть $E \subset Z$ - исключительный дивизор раздутия $\varphi$, т. е. $E=\varphi^{-1}(R)$. Очевидно, что проекция $\mathbb{F}_{1} \rightarrow \mathbb{P}^{1}$ (регуляризованная проекция $\mathbb{P}^{2}$ из точки $R \cap \pi^{-1}(s)$, $s \in S)$ порождает проекцию $p: Z \rightarrow E$, которая является $\mathbb{P}^{1}$-расслоением, т. е. $Z / E$ - расслоение Мори (Фано). Это дает диаграмму (5) линка первого типа с $T=E$, где $\varepsilon: T \rightarrow S$ есть проекция $\varphi: E \rightarrow R$ относительно отождествления $\pi: R \rightarrow S$ и $Z=Y, \psi$ - тождественное отображение. В описанном примере расслоение Мори $Y / T$ получается из $X / S$ путем расслоения слоев $\pi^{-1}(s)$, $s \in S$. Соответственно, слои нового расслоения $Y / T$ имеют меньшую размерность. Эту операцию можно интерпретировать и как расслоение общего слоя $F_{\eta}$ морфизма $X \rightarrow S$ над прямой $\mathbb{P}_{\eta}^{1}$ (общим слоем морфизма $T \rightarrow S$ ).

Тип II (послойные перестройки). В этом случае $S=T$. Имеются экстремальные дивизориальные стягивания $\varphi: Z \rightarrow X$ и $\lambda: W \rightarrow Y$ и бирациональный изоморфизм $\psi: Z \rightarrow W$, являющийся композицией флопов (изоморфизм в коразмерности единица) такие, что коммутативна диаграмма

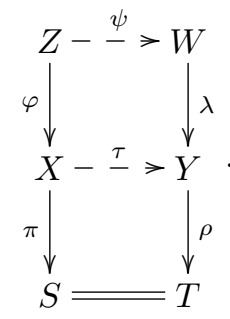

Рассмотренные выше (пример 1.4) послойные бирациональные автоморфизмы пучков кубических поверхностей принадлежат к этому типу.

Рассмотрим еще один пример.

Пример 1.9 (замена слоя). Пусть $S$ - гладкая кривая, $\pi: X \rightarrow S-\mathbb{P}^{n}$-расслоение, $x \in X-$ произвольная точка, $F=\pi^{-1}(\pi(x)) \cong \mathbb{P}^{n}-$ содержащий ее слой. Пусть

$$
\varphi: Z \rightarrow X
$$

- раздутие точки $x, E=\varphi^{-1}(x) \cong \mathbb{P}^{n}$ - исключительный дивизор, $F^{+} \subset Z$ собственный прообраз слоя $F$. Очевидно, что $R=F^{+} \cap E$ есть гиперплоскость в $E=\mathbb{P}^{n}$ и проекция слоя $F=\mathbb{P}^{n}$ из точки $x$ порождает $\mathbb{P}^{1}$-расслоение

$$
\lambda_{F}: F^{+} \rightarrow R
$$

Если $L \subset F$ - произвольная прямая, проходящая через точку $x$, то ее собственный прообраз $L^{+} \subset F^{+}$есть слой проекции $\lambda_{F}$. Поскольку $K_{Z}=\varphi^{*} K_{X}+n E$, имеют место равенства

$$
\left(K_{Z} \cdot L^{+}\right)=-1, \quad\left(F^{+} \cdot L^{+}\right)=-1
$$

(с учетом того, что $\left.\left(K_{X} \cdot L\right)=-(n+1)\right)$, так что численный класс кривой $L^{+}$ порождает экстремальный луч $\left[L^{+}\right] \in \overline{N E}(Z)$. Пусть $\lambda: Z \rightarrow Y-$ стягивание 
этого луча. Очевидно, что $\left.\lambda\right|_{F^{+}}=\lambda_{F}$ и $\lambda$ стягивает дивизор $F^{+}$. Образ $Y$ есть снова $\mathbb{P}^{n}$-расслоение над $S$, бирационально изоморфное исходному:

$$
\tau=\lambda \circ \varphi^{-1}: X \rightarrow Y
$$

есть линк второго типа. Здесь $W=Z$ и $\psi$ - изоморфизм. Расслоение $Y / S$ имеет те же самые слои над всеми точками кривой $S$, за исключением точки $\pi(x) \in S$. Слой над этой точкой заменяется на исключительный дивизор $E=\mathbb{P}^{n}$. При $n=1$ описанная процедура есть классическая перестройка линейчатой поверхности.

Наконец, бирациональная перестройка расслоения на коники, описанная в примере 1.5, также относится к типу II. (На самом деле, линки этого типа смоделированы с этих перестроек.) Отметим, что по построению линк типа II всегда индуцирует бирациональный изоморфизм общих слоев $F_{\eta} \rightarrow G_{\eta}$ расслоений Мори $X / S$ и $Y / S$.

Tип III (сужение базы). Линки этого типа обратны линкам типа I, т. е. в диаграмме (5) левая и правая стороны меняются местами. Точнее, имеются: экстремальное дивизориальное стягивание $\varphi: Z \rightarrow Y$, бирациональное отображение $\psi: X \rightarrow Z$, являющееся композицией флопов (изоморфизм в коразмерности единица) и экстремальное стягивание $\varepsilon: S \rightarrow T, \rho(S / T)=1$, такие, что коммутативна диаграмма отображений

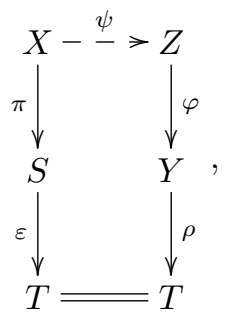

причем $\varphi \circ \psi=\tau: X \rightarrow Y$. Обращая конструкцию примера 1.5, получаем линк типа III. С геометрической точки зрения, наиболее интересно, что композиция двух линков, имеющих тип I и тип III, приводит, вообще говоря, к бирациональной перестройке, не согласованной со структурами расслоения, т. е. слои не переводятся в слои, как показывает следующий пример.

ПримеР 1.10. В обозначениях примера 1.8 выберем сечение $R \subset X$ специальным образом: пусть $R=\sigma^{-1}\left(L^{*}\right)$, где $L^{*} \subset \mathbb{P}^{3}-$ прямая, не пересекающая $L$, в частности, $\left\langle L, L^{*}\right\rangle=\mathbb{P}^{3}$. В этом случае композиция $\sigma \circ \varphi: Z \rightarrow \mathbb{P}^{3}$ есть раздутие гладкой приводимой (несвязной) кривой $L \cup L^{*}$ с исключительным дивизором $E_{L} \cup E$, где $E_{L}=\sigma^{-1}(L)$ - исключительный дивизор раздутия $\sigma$. Стягивая $E_{L} \subset Z$, получаем линк типа III

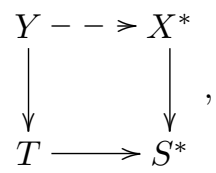


где $X^{*}$ есть $\mathbb{P}^{3}$ с раздутой прямой $L^{*}$, кривая $S^{*}$ есть $\mathbb{P}^{1}$ и $X^{*} \rightarrow S^{*}$ есть регуляризированная проекция из $L^{*}$. Композиция этого линка с линком примера 1.8 дает бирациональное отображение

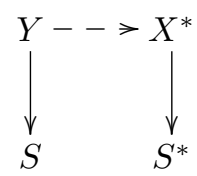

$\mathbb{P}^{2}$-расслоений над $S, S^{*} \cong \mathbb{P}^{1}$, не согласованное со структурами этих расслоений. Отметим, что в рассматриваемом специальном случае

$$
T=S \times S^{*}=\mathbb{P}^{1} \times \mathbb{P}^{1}
$$

и проекция $Y \rightarrow T$ есть регуляризация рационального отображения

$$
\mathbb{P}^{3} \rightarrow L \times L^{*},
$$

сопоставляющего точке $x \in \mathbb{P}^{3} \backslash\left(L \cup L^{*}\right)$ единственную пару точек $\left(z, z^{*}\right) \in$ $L \times L^{*}$ такую, что $x \in\left\langle z, z^{*}\right\rangle$ (прямые $L$ и $L^{*}$ естественно отождествляются с кривыми $S^{*}$ и $S$ как сечения расслоений Мори $X^{*} / S^{*}$ и $X / S$ соответственно). Отметим также, что диаграмма

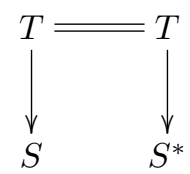

есть пример линка типа IV (в размерности два), к описанию которого мы переходим.

Tип IV (переход $к$ новой структуре расслоения). Этот тип наиболее интересен. Имеются: бирациональное отображение $\psi: X \rightarrow Y$, являющееся композицией флопов, и экстремальные стягивания $s: S \rightarrow R$ и $t: T \rightarrow R$ такие, что коммутативна диаграмма отображений

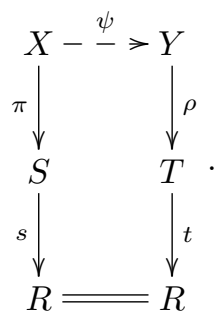

Простейший пример линка последнего типа приведен выше (переход от одной проекции $\mathbb{P}^{1} \times \mathbb{P}^{1} \rightarrow \mathbb{P}^{1}$ к другой). Нетривиальный пример линка типа IV описан в примере 1.7. В этом примере многообразие $V$ обладает ровно двумя структурами расслоения Фано, а переход от одной структуры к другой осуществляется через флоп.

K настоящему моменту наиболее сильный доказанный факт о разложении бирациональных отображений в композицию линков - это следующая теорема. 
Теорема 1.3 [11]. Пусть $\pi: X \rightarrow S u \pi^{\prime}: X^{\prime} \rightarrow S^{\prime}-$ расслоения Мори c $\mathbb{Q}$-бакториальными терминальными особенностями. Многообразия $X$ и $X^{\prime}$ бирационально эквивалентны тогда и только тогда, когда существует последовательность линков

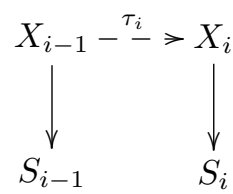

$(i=1, \ldots, N)$, связывающая $X / S$ и $X^{\prime} / S^{\prime}$, m.e.

$$
X_{0} / S_{0}=X / S \quad \text { u } \quad X_{N} / S_{N}=X^{\prime} / S^{\prime}
$$

Однако, как было сказано выше, основной вопрос заключается в том, можно ли любое бирациональное отображение $\chi: X \rightarrow X^{\prime}$ разложить в композицию линков. В размерности три ответ положительный [12], в размерности $\geqslant 4$ верно более слабое утверждение, сформулированное в теореме. Это связано с различными подходами к задаче факторизации бирациональных отображений.

2.3. О доказательстве программы Саркисова. Опишем кратко исходный подход Саркисова [13], [14], реализованный в [12]. Пусть $\chi: X \rightarrow X^{\prime}-$ бирациональное отображение между пространствами расслоений Мори $X / S$ и $X^{\prime} / S^{\prime}$ размерности три. Необходимо показать, что $\chi$ раскладывается в композицию элементарных линков, $\chi=\tau_{N} \cdots \tau_{1}$. Доказательство, основанное на исходных идеях Саркисова, состоит в предъявлении индуктивного алгоритма, сопоставляющего бирациональному отображению $\chi$, а точнее, диаграмме

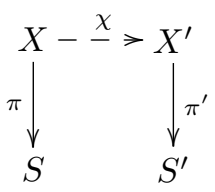

откручивающий линк одного из типов I-IV,

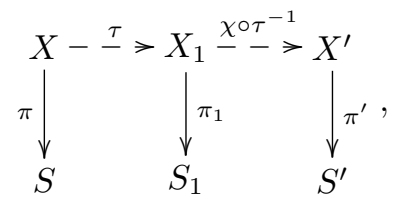

понижающий некоторый инвариант исходного бирационального отображения,

$$
\delta\left(\chi \circ \tau^{-1}\right)<\delta(\chi)
$$

который не может понижаться до бесконечности.

Опишем этот инвариант. Размерность $\operatorname{dim} X$ считаем произвольной $\geqslant 3$. Поскольку $\rho\left(X^{\prime} / S^{\prime}\right)=1$, найдутся целое положительное число $m$ и очень обильный дивизор $A^{\prime}$ на базе $S^{\prime}$ такие, что

$$
D^{\prime}=-m K_{X}+\pi^{* *} A^{\prime}
$$


- очень обильный дивизор на $X^{\prime}$. Очень обильная линейная система $\left|D^{\prime}\right|=\mathscr{H}^{\prime}$ фиксирована на протяжении всего процесса факторизации отображения $\chi$. Отметим, что отображение $\chi$ является послойным (т. е. существует рациональное доминантное отображение $\varepsilon: S \rightarrow S^{\prime}$, превращающее диаграмму (6) в коммутативную, $\left.\varepsilon \pi=\pi^{\prime} \chi\right)$ тогда и только тогда, когда собственный прообраз линейной системы $\left|\pi^{\prime *} A^{\prime}\right|$ поднят с базы $S$, т. е.

$$
\chi_{*}^{-1}\left|\pi^{\prime *} A^{\prime}\right| \subset\left|\pi^{*} A^{+}\right|
$$

для некоторого (подвижного) дивизора $A^{+}$на базе $S$. Пусть

$$
\mathscr{H}=\chi_{*}^{-1}\left|D^{\prime}\right|
$$

- собственный прообраз системы $\Sigma^{\prime}$ на $X$. Поскольку $X / S$ - расслоение Мори, имеем

$$
\mathscr{H} \subset\left|-n K_{X}+\pi^{*} A\right|
$$

для некоторого $\mathbb{Q}$-Картье дивизора $A$ на $S$. В силу ограниченности знаменателей можно считать, что $n \in \mathbb{Z}_{+}$. Очевидно, что $n \geqslant 1$. Имеет место следующее утверждение.

ПРЕДЛОЖЕНИЕ 1.1. Справедливо неравенство $n \geqslant m$. Если $n=m$, то отображение ұ является послойным.

ДоказАтельство (см., например, [12]) элементарно. Оно почти дословно повторяет рассуждения, использованные в доказательстве предложения 2.6 предыдущего обзора [1]. Наметим его: пусть $\varphi: Z \rightarrow X-$ разрешение особенностей отображения $\chi, \psi=\chi \circ \varphi: Z \rightarrow X^{\prime}$ - сквозное отображение (бирациональный морфизм), а

$$
\left\{E_{i} \mid i \in I\right\} \quad \text { и } \quad\left\{E_{j}^{\prime} \mid j \in J\right\}
$$

- множества $\varphi$ - и $\psi$-исключительных дивизоров соответственно. Повторяя дословно рассуждения из [1; предложение 2.6$]$, имеем

$$
\left(1-\frac{n}{m}\right) \varphi^{*} K_{X}+\frac{1}{m} \varphi^{*} \pi^{*} A=\frac{1}{m} \psi^{*} \pi^{\prime *} A^{\prime}+\sum_{j \in J} a_{j}^{\prime} E_{j}^{\prime}+\sum_{i \in I}\left(\frac{b_{i}}{m}-a_{i}\right) E_{i},
$$

где $a_{j}^{\prime}>0, a_{i}>0$ (особенности терминальны) и $b_{i} \geqslant 0$. Поскольку $E_{i}$ являются $\varphi$-исключительными, ограничение этого равенства на слой общего положения проекции $\pi$ показывает, что $n \geqslant m$, причем если $n=m$, то собственный прообраз линейной системы $\left|\pi^{\prime *} A^{\prime}\right|$ относительно $\chi$ поднят с базы $S$, а каждый $\psi$-исключительный дивизор $E_{j}^{\prime}$ либо является $\varphi$-исключительным, либо его образ $\varphi\left(E_{j}^{\prime}\right)$ поднят с базы $S$. Этим предложение 1.1 доказано.

Напомним [12], что каноническим (соответственно логканоническим) порогом пары $(X, \mathscr{H})$ называется число

$$
\operatorname{ct}(X, \mathscr{H})=\sup \left\{\alpha \in \mathbb{Q}_{+} \mid \text {пара }(X, \alpha \mathscr{H}) \text { канонична }\right\}
$$


(соответственно $\operatorname{lct}(X, \mathscr{H})=\sup \left\{\alpha \in \mathbb{Q}_{+} \mid\right.$пара $(X, \alpha \mathscr{H})$ логканонична $\left.\}\right)$. Если $\varphi: Z \rightarrow X$ - разрешение особенностей пары $(X, \mathscr{H})$ с множеством исключительных дивизоров $\left\{E_{i} \mid i \in I\right\}$, то

$$
\frac{1}{\operatorname{ct}(X, \mathscr{H})}=\max _{i \in I} \frac{\operatorname{ord}_{E_{i}} \varphi^{*} D}{a\left(E_{i}, X\right)}
$$

для общего дивизора $D \in \mathscr{H}$ и аналогичным образом

$$
\frac{1}{\operatorname{lct}(X, \mathscr{H})}=\max _{i \in I} \frac{\operatorname{ord}_{E_{i}} \varphi^{*} D}{a\left(E_{i}, X\right)+1} .
$$

В частности, $\operatorname{lct}(X, \mathscr{H})>\operatorname{ct}(X, \mathscr{H})$. Если $\operatorname{ct}(X, \mathscr{H})=\gamma$, то для каждого исключительного дивизора $E_{i}$ справедливо неравенство

$$
\gamma \operatorname{ord}_{E_{i}} \varphi^{*} \mathscr{H} \leqslant a\left(E_{i}, X\right)
$$

При этом для хотя бы одного дивизора $E_{i}$ это неравенство является равенством. Такие дивизоры называются крепантными дивизорами; соответствующие им дискретные нормирования поля рациональных функций $\mathbb{C}(X)$ не зависят от выбора разрешения и определяются парой $(X, \mathscr{H})$. Пусть $e(X, \mathscr{H}) \geqslant 1$ - число крепантных нормирований.

ОПРЕДЕЛЕНИЕ 1.2. Степенъю (или степенъю Саркисова) пары $(X, \mathscr{H})$ называется тройка чисел

$$
\delta(X, \mathscr{H})=(n, \gamma=\operatorname{ct}(X, \mathscr{H}), e=e(X, \mathscr{H})) .
$$

На множестве значений степени $\delta$ вводится следующее отношение порядка (соответствующее лексикографическому упорядочению троек $\left.\left(n, \gamma^{-1}, e\right)\right)$ :

$$
\delta=(n, \gamma, e)>\delta_{1}=\left(n_{1}, \gamma_{1}, e_{1}\right),
$$

если либо $n_{1}<n$, либо $n_{1}=n$, но $\gamma_{1}>\gamma$, либо, наконец, $n_{1}=n$ и $\gamma_{1}=\gamma$, но $e_{1}<e$. Поскольку (при фиксированной очень обильной системе $\mathscr{H}^{\prime}$ ) отображение $\chi$ однозначно определяется системой $\mathscr{H}$, можно писать $\delta(\chi)$ вместо $\delta(X, \mathscr{H})$. На этом этапе в программе Саркисова появляется ключевое понятие максимальной особенности.

ОПРЕДЕЛЕНИЕ 1.3. Максимальной особенностью линейной системы $\mathscr{H}$ (или бирационального отображения $\chi$ ) в смысле программы минимальных моделей (короче, ПММ-максимальной особенностью) называется исключительный дивизор $E \subset Z$ некоторого разрешения $\varphi: Z \rightarrow X$ пары $(X, \mathscr{H})$ (или соответствующее ему дискретное нормирование поля рациональных функций $\mathbb{C}(X))$, если выполнено неравенство Нётера-Фано

$$
\operatorname{ord}_{E} \varphi^{*} \mathscr{H}>n a(E, X) .
$$

В [12] вместо термина "максимальная особенность" используется "базисная компонента высокой кратности". В обзоре [15] возвращена "максимальная особенность", однако необходимо помнить, что в классической теории параметр $n$ 
есть порог канонического присоединения. В рассматриваемой выше общей ситуации $c(\mathscr{H})=n$, только если дивизор $A$ псевдоэффективен на базе $S$. Таким образом, между понятиями максимальной особенности в традиционном подходе и в ПММ имеются некоторые различия (проигнорированные в [12]). Чтобы обратить на это внимание, мы говорим о ПММ-максимальных особенностях (для большинства решенных к настоящему времени задач эти различия несущественны).

Очевидно, что ПММ-максимальная особенность существует, если и только если выполнено неравенство

$$
\operatorname{ct}(X, \mathscr{H})<\frac{1}{n}
$$

Далее [12; теорема 5.4], если $\mathscr{H}$ имеет ПММ-максимальную особенность, то применение $\left(K_{Z}+\alpha \mathscr{H}_{Z}\right)$-ПММ к экстремальному раздутию $\varphi: Z \rightarrow X$ одного из крепантных дискретных нормирований, где число $\alpha$ специально подбирается, приводит к линку $\tau: X / S \rightarrow X_{1} / S_{1}$ типа I или II такому, что $\left(n_{1}, \gamma_{1}, e_{1}\right)<(n, \gamma, e)$, причем если $n_{1}=n$, то $\tau$ является послойным и индуцирует бирациональные изоморфизмы баз $S \rightarrow S_{1}$ и общих слоев,

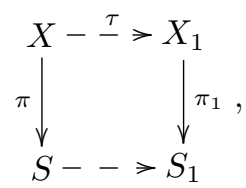

т. е. является "квадратом" в терминологии [12]. Если же $\mathscr{H}$ не имеет ПММ-максимальных особенностей (т. е. выполнено неравенство $\operatorname{ct}(X, \mathscr{H}) \geqslant 1 / n)$, то строится последовательность линков

$$
X / S-\stackrel{\tau_{1}}{-}>X_{1} / S_{1}-\stackrel{\tau_{2}}{-}>\cdots-\stackrel{\tau_{k}}{-}>X_{k} / S_{k}
$$

типа III и IV такая, что либо $n=n_{1}=\cdots=n_{k-1}>n_{k}$, либо $n=n_{1}=\cdots=n_{k}$ и индуцированное бирациональное отображение есть изоморфизм расслоений Мори. Наконец, последний необходимый факт - конечность этой процедуры, т. е. решение задачи факторизации за конечное число шагов - доказывается с помощью теоремы Алексеева об отсутствии точек накопления снизу для логканонических порогов [16]. Полное доказательство теоремы о факторизации см. в [12] или в обзоре [15].

Описанный выше алгоритм в принципе не зависит от размерности, однако не все факты ПММ, нужные для его обоснования, доказаны сегодня в размерности $\geqslant 4$ (хотя ведется работа и имеется существенный прогресс - например, по поводу обрыва для порогов см. [17], [18]). Все эти факты суть некоторые утверждения о конечности (например, обрыв последовательности флипов), они нужны как для построения линков, так и для обоснования конечности процедуры факторизации. Используя оказавшийся столь успешным в [10] подход, 
K. Хэкон и Дж. Маккернан в [11] доказали ослабленный вариант программы Саркисова в произвольной размерности: вместо того чтобы строить алгоритм факторизации и доказывать его конечность, они установили существование некоторой последовательности линков, композиция которых дает бирациональный изоморфизм $X \rightarrow X^{\prime}$ пространств расслоений Мори $X / S$ и $X^{\prime} / S^{\prime}$ (про которые заранее предполагается, что они бирационально изоморфны).

Основной результат [11] имеет следующий вид.

Теорема 1.4. Предположим, что расслоения Мори $X / S$ и $X^{\prime} / S^{\prime}$ являются

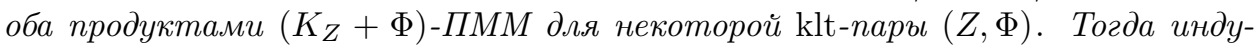
ицрованное бирациональное отображение $X \rightarrow X^{\prime}$ есть композичия линков muпов I-IV.

Необходимо отметить, что если пространства расслоений Мори бирационально эквивалентны и имеют $\mathbb{Q}$-факториальные терминальные особенности, то они являются продуктами $K_{Z}$-ПММ для некоторого многообразия $Z$, так что соответствующие расслоения Мори удовлетворяют предположениям теоремы 1.4.

Доказательство теоремы 1.4 основано на технике, развитой в [10]; к последней работе мы и отсылаем заинтересованного читателя.

\section{4. Метод максимальных особенностей и теория факторизации:} сопоставление. После всего сказанного имеет смысл сравнить два подхода к изучению бирациональной геометрии рационально связных расслоений: классический метод максимальных особенностей и программу факторизации бирациональных отображений, основанную на теории минимальных моделей (программу Саркисова). Следует отметить, что оба подхода восходят к одним и тем же идеям (развитым в работах М. Нётера и его предшественников, а затем в работах итальянских классиков вплоть до Фано, см. [1]) и потому во многих отношениях достаточно близки. Между этими подходами имеются, однако, и существенные различия, на которых мы сейчас и остановимся.

Основная идея метода максимальных особенностей, описанная в подробностях в [1], состоит в изучении максимальных особенностей бирационального отображения $\chi: V \rightarrow V^{\prime}$ или, что эквивалентно, максимальных особенностей подвижной линейной системы $\Sigma$ на $V$, задающей это бирациональное отображение. Метод успешно работает, если удается явно описать потенциальные максимальные особенности, которые затем откручиваются - как правило, бирациональными автоморфизмами $\tau \in \operatorname{Bir} V$ (в относительном случае - послойными бирациональными автоморфизмами $\tau \in \operatorname{Bir}(V / S))$. Вопрос о том, почему почти все потенциальные максимальные особенности можно открутить автоморфизмами, не обсуждается, это - эмпирический факт. В тех успешно исследованных случаях, когда автоморфизмов недостаточно, каждая максимальная особенность явно определяет переход к другой структуре расслоения Фано. Метод максимальных особенностей смоделирован с пионерской работы [19]; за прошедшие почти сорок лет общий подход мало изменился, хотя техническая сторона метода радикально усилена и преобразована. 
ПММ-подход смоделирован с доказательства теоремы Саркисова [2] с учетом идеологии теории Мори. Этот подход примерно на пятнадцать лет "моложе". Его основная идея, описанная в $\S 1$, состоит в упрощении бирационального отображения (или подвижной линейной системы) с помощью элементарных линков. Линк строится применением ПММ к подходящей логпаре. Априори линк является бирациональным отображением между различными расслоениями Мори, т. е. даже в случае послойного линка происходит переход на другую модель.

Метод максимальных особенностей - это (технически мощная и успешно работающая) схема рассуждений, приводящая к очень сильным индивидуальным результатам, т. е. результатам для конкретных явно заданных семейств многообразий и расслоений Фано. Напротив, программа Саркисова (в той форме, в которой она известна с появления работы [12]) - это общая теорема существования, утверждающая сам факт возможности профакторизовать бирациональное отображение (или несколько более слабый факт в размерности $\geqslant 4)$. Каждый из этих двух подходов имеет преимущества и недостатки.

Несомненным достоинством программы Саркисова является ее общность. Из описания программы и доказательства основной теоремы следует некоторая процедура построения факторизующих линков. Учитывая быстрое развитие ПММ с середины 80-х годов, неудивительно, что доказательство программы Саркисова в [12] стало источником большого оптимизма в отношении трехмерной бирациональной геометрии. В 1993-95 гг. многим казалось, что решение задач классической бирациональной геометрии в размерности три даже таких, как описание группы Кремоны ранга 3, или описание группы бирациональных автоморфизмов трехмерной кубики, или доказательство критерия рациональности трехмерных расслоений на коники - дело недалекого будущего. Некоторые высказывания на эту тему (хотя и в весьма осторожной форме) можно найти в [12]. Речь шла, в первую очередь, о передоказательстве известных результатов, полученных методом максимальных особенностей, а затем и о дальнейшем прогрессе там, где классические методы уже не приводили к успеху.

Сегодня по прошествии более пятнадцати лет, можно сказать, что этим надеждам не суждено было сбыться. Задачи описания бирациональных отображений между трехмерными расслоениями Мори оказались гораздо сложнее, чем предполагали авторы [12], [14]. Их оптимизм начала 90-х годов объясняется, скорее, слабым знакомством с предшествующими работами московской школы бирациональной геометрии; вероятно, по той же причине в [12] работы московской школы отодвигаются в прошлое - ожидалось, что новая техника ПММ позволит легко преодолеть трудности, препятствовавшие успешной работе метода максимальных особенностей. Следует признать, что некоторые идеи, пришедшие из ПММ, оказались действительно плодотворными. Речь идет, прежде всего, о так называемом принципе связности Шокурова-Коллара и вытекающем из него обращении присоединения (см. п. 3.3 настоящей главы и $\S 2$ главы 3), которые позволили существенно упростить некоторые звенья классической техники (ср., например, исключение максимальной особенности 
над квадратичной точкой в [20] и в [21] - при последнем подходе по сути ничего, кроме непосредственного применения обращения присоединения, не требуется).

Однако в целом попытки реализовать программу Саркисова для широкого класса трехмерных расслоений Мори не были успешными. Изучение бирациональных отображений между конкретными трехмерными многообразиями в большинстве случаев следовало проторенной дорогой классического метода максимальных особенностей, иногда с некоторыми техническими усовершенствованиями. Это касается уже самой первой, и наиболее известной, работы этой серии [22], изначально задуманной как первый широкомасштабный этап в реализации трехмерной программы Саркисова. Оказалось, что техника ПММ для всех 95 типов взвешенных гиперповерхностей Фано бесполезна, потому что все потенциальные максимальные особенности (на многих многообразиях их просто нет) откручиваются бирациональными автоморфизмами, которые, в свою очередь, строятся с помощью испытанной классической техники. Тем не менее, работа [22] интерпретировалась как результат применения новой ПММ-техники (см., например, обзор [15]), что, конечно, было большим преувеличением. Статья [22] написана на новом языке (и содержит новые доказательства некоторых известных фактов классической теории), но это, пожалуй, самое большее, что можно сказать. По своему содержанию [22] безусловно принадлежит к серии работ, сделанных методом максимальных особенностей, и непосредственно примыкает к статье [19] о трехмерной квартике.

Кроме [22], было опубликовано еще несколько работ, где схема программы Саркисова применялась для изучения бирациональных отображений между трехмерными расслоениями Мори (см., прежде всего [23], [24]). В [21] на языке программы Саркисова излагаются некоторые известные результаты, ранее полученные методом максимальных особенностей. В [23], [24] эта программа действительно работает - строятся нетривиальные линки, связывающие изучаемые многообразия с другими расслоениями Мори, однако существование такого линка, хотя и означает, в терминологии Рида-Корти, что многообразие не является бирационально жестким, тем не менее не дает сколько-нибудь значительной информации о его бирациональном типе. Примыкающая к этой серии работ статья [25] содержит нетривиальные вычисления и конструкции, а также ряд интересных гипотез, но не дает никакого продвижения в направлении их доказательства.

Скудость результатов, полученных в ходе попыток применить общую теорию [12] к изучению бирациональной геометрии трехмерных расслоений Мори, тем более впечатляет, если сравнить их с результатами, полученными за тот же период в рамках классического метода максимальных особенностей [3], [26]-[29], не говоря о результатах в произвольной размерности. Причиной такой неуспешности является, по-видимому, тот факт, что для построения линков требуется точное описание всех потенциальных максимальных особенностей, так что работа классического метода максимальных особенностей неизбежно включается в работу программы Саркисова как наиболее важный этап. С другой стороны, имеет место уже упоминавшийся эмпирический факт - для почти всех (доступных для изучения) многообразий потенциально реализуемые максимальные особенности откручиваются бирациональными автоморфизма- 
ми, что делает излишней технику ПММ: бирациональное отображение между заданными многообразиями достаточно определить в общей точке, не заботясь о том, как его разложить в последовательность экстремальных стягиваний/раздутий и флипов. Этими соображениями, по-видимому, и объясняется крайне низкая эффективность общей теории [12], за пятнадцать лет почти не нашедшей себе применения.

В качестве иллюстрации обратимся к примеру 1.4. Пусть $V \stackrel{\pi}{\rightarrow} \mathbb{P}^{1}-$ стандартное расслоение на кубические поверхности, $C \subset V$ - сечение проекции $\pi$. Предположим, что $C$ является максимальной кривой подвижной линейной системы $\Sigma \subset\left|-n K_{V}+l F\right|$, где $l \in \mathbb{Z}_{+}$. Экстремальное раздутие, связанное с кривой $C$, есть просто ее раздутие в обычном понимании, $\varphi: \widetilde{V} \rightarrow V$. Теперь общая теория гарантирует, что применение ПММ к подходящей паре $(\widetilde{V}, \alpha \widetilde{\Sigma})$ приводит к линку $V \rightarrow V^{+} / \mathbb{P}^{1}$, откручивающему максимальную кривую $C$. Однако это общее утверждение, использующее всю мощь ПММ, практически бесполезно, поскольку конструкция линка очевидна из элементарных геометрических соображений, и ее обоснование не требует усилий, так как $V=V^{+}$и линк корректно определен на общем слое. Имеется конечное множество слоев $F_{i}=\pi^{-1}\left(t_{i}\right)$, $i=1, \ldots, k$, где через точку $C \cap F_{i}$ проходит по крайней мере одна прямая на $F_{i}$. ПММ гарантирует, что некоторая последовательность флопов, начинающаяся с $\widetilde{V}$, приводит к модели $V^{\sharp}$, допускающей экстремальное стягивание (так что бирациональный автоморфизм примера 1.4 , связанный с сечением $C$, является композицией двух линков - типа I и типа III), однако классическая техника с гораздо меньшими усилиями сразу дает окончательный результат. Особенно важно при этом, что, используя классический подход, мы остаемся на той же модели, в то время как общая теория обеспечивает откручивание кривой $C$, вообще говоря, лишь переходом на некоторое новое расслоение Мори. Поэтому, если классический метод максимальных особенностей требует лишь информации о бирегулярной геометрии данной модели расслоения Мори, эффективная работа программы Саркисова требует (как, впрочем, и естественно ожидать от теории такой степени общности) информации о всех моделях данной размерности. Бирегулярная классификация расслоений Мори даже в размерности три еще очень далека от завершения (в каком-либо разумном смысле). Поэтому общая теория [12] столь неэффективна.

В размерности три имеются три класса расслоений Мори: многообразия Фано (с $\mathbb{Q}$-факториальными терминальными особенностями), расслоения на поверхности дель Пеццо и расслоения на коники. К середине 90-х годов о бирациональной геометрии многообразий первого и третьего класса было известно достаточно много, однако расслоения на поверхности дель Пеццо оставались белым пятном. Попытки изучить их геометрию классическим методом с помощью техники пробного класса предпринимались около десяти лет, но оказались безуспешными [30] (как выяснилось немного позднее [3], это было невозможно в принципе). Положение радикально изменилось с появлением работы [3], в которой классический подход был существенно переосмыслен, в частности, техника пробного класса была заменена техникой подсчета кратностей. Однако, насколько известно автору, вплоть до недавнего времени предпринимались попытки получить результаты о бирациональной геометрии расслоений на по- 
верхности дель Пеццо методами, аналогичными доказательству теоремы Саркисова о расслоениях на коники (пример 1.5), т. е. путем послойных перестроек. (Поскольку именно максимальные особенности, центр которых содержится в слое, представляют наибольшую трудность - см. главу 2.) Эти попытки также были безуспешными. В отличие от расслоений на коники, даже простейшие послойные перестройки пучка кубических поверхностей приводят к многообразиям со сложными особенностями и работа на этом останавливается.

Таково положение на сегодняшний день; привлечение новых идей и новых фактов в будущем может, конечно, изменить эту ситуацию.

Подчеркнем, что данный параграф ни в какой мере не является обзором самой программы минимальных моделей или программы Саркисова. По этой причине мы не упоминаем (и, тем более, не обсуждаем) основных работ по ПММ (Мори, Каваматы, Шокурова, Коллара и многих других), за исключением статьи [10]. На этом мы завершаем краткий экскурс в общую теорию факторизации бирациональных отображений (программу Саркисова) и возвращаемся к теории, технике и результатам метода максимальных особенностей предмету настоящего обзора.

\section{§ 3. Бирациональная жесткость расслоений Фано}

3.1. Порог канонического присоединения. Для произвольного рационально связного гладкого проективного многообразия $X$ символом $A^{i} X$ обозначим группу Чжоу алгебраических циклов коразмерности $i \geqslant 1$ по модулю численной эквивалентности, $A_{\mathbb{R}}^{i} X=A^{i} X \otimes \mathbb{R}$. Символом $A_{+}^{i} X$ обозначим замкнутый конус в $A_{\mathbb{R}}^{i} X$, порожденный классами эффективных циклов (псевдоэффективный конус). Символом $A_{\text {mov }}^{i} X$ обозначим замкнутый конус в $A_{\mathbb{R}}^{i} X$, порожденный классами подвижных дивизоров (т. е. таких дивизоров $D$, что полная линейная система $|D|$ не имеет неподвижных компонент) - подвижный конус.

Рассмотрим стандартное расслоение Фано $\pi: V \rightarrow S$. Очевидно, имеем вложение $\pi^{*} A_{\mathrm{mov}}^{i} S \subset A_{\mathrm{mov}}^{i} V$. Далее,

$$
A^{1} V=\mathbb{R}\left[K_{V}\right] \oplus \pi^{*} A^{1} S .
$$

ОПРЕДЕЛЕниЕ 1.4. Скажем, что стандартное расслоение Фано $\pi: V \rightarrow S$ удовлетворяет $K$-условию, если

$$
A_{\mathrm{mov}}^{1} V \subset \mathbb{R}_{+}\left[-K_{V}\right] \oplus \pi^{*} A_{+}^{1} S .
$$

Иными словами, $V / S$ удовлетворяет $K$-условию тогда и только тогда, когда для любой подвижной линейной системы $\left|-n K_{V}+\pi^{*} A\right|$ класс $A \in \operatorname{Pic} S$ псевдоэффективен. Если псевдоэффективный конус $A_{+}^{1} S$ устроен достаточно просто (например, $A^{1} S=\mathbb{Z} H_{S}$, где $H_{S}$ - обильная образующая, так что $A_{+}^{1} S=\mathbb{R}_{+}\left[H_{S}\right]-$ положительный луч, или $S=S_{1} \times \cdots \times S_{k}$, где $\left.A^{1} S_{i} \cong \mathbb{Z}\right)$, то нетрудно проверить, что, в некотором смысле, "подавляющее большинство" стандартных расслоений Фано с данной фиксированной базой $S$ этому условию удовлетворяет. В качестве иллюстрации рассмотрим конструкцию примера 1.1, предполагая, что $A^{1} S=\mathbb{Z} H_{S}$. 
В обозначениях примера 1.1 имеем $A_{i} \sim a_{i} H_{S}$ для некоторых $a_{i} \in \mathbb{Z}$. Положим $a=a_{1}+\cdots+a_{k}$. Подкручивая локально свободный пучок $\mathscr{E}$, можно считать, что он порожден глобальными сечениями, так что тавтологический класс $L_{X}$ численно эффективен. Положим также

$$
b H_{S} \sim \operatorname{det} \mathscr{E}+K_{S},
$$

где целочисленный параметр $b$ зависит только от $X$. Имеем

$$
K_{V}=-L+(a+b) H,
$$

где для сокращения записи мы положили $L=L_{V}$ и $H=\pi^{*} H_{S}$. Теперь если линейная система $\left|-n K_{V}+l H\right|$ непуста (тем более подвижна), то имеет место неравенство

$$
\begin{array}{r}
\left(-n K_{V}+l H\right) \cdot L^{M} \cdot H^{\operatorname{dim} S-1} \geqslant 0, \\
\| \\
n L^{M+1} H^{\operatorname{dim} S-1}+d(l-n(a+b)) H^{\operatorname{dim} S}
\end{array}
$$

где $d=d_{1} \cdots d_{k}$, откуда сразу вытекает, что для некоторого $a_{0} \in \mathbb{Z}$ при $a \geqslant a_{0}$ имеем $l \in \mathbb{Z}_{+}$, т. е. $K$-условие выполнено для всех стандартных расслоений Фано, удовлетворяющих неравенству $a_{1}+\cdots+a_{k} \geqslant a_{0}$.

Этот пример показывает, что “большинство" стандартных расслоений Фано примера 1.1 удовлетворяет более сильному условию:

$$
A_{+}^{1} V \subset \mathbb{R}_{+}\left[-K_{V}\right] \oplus \pi^{*} A_{+}^{1} S .
$$

Ситуация аналогична для двойных накрытий Фано (пример 1.2). Наоборот, легко увидеть, что прямые произведения Фано (пример 1.3) не удовлетворяют $K$-условию.

Рассмотрим теперь наиболее интересный случай $S=\mathbb{P}^{1}$. Для проверки $K$-условия воспользуемся тем, что самопересечение подвижного класса $z \in$ $A_{\text {mov }}^{1} X$ есть псевдоэффективный класс коразмерности два: $z^{2} \in A_{+}^{2} X$. Таким образом, если $z^{2} \notin A_{+}^{2} X$, то $z \notin A_{\mathrm{mov}}^{1} X$.

ОПРедЕлЕниЕ 1.5. Скажем, что стандартное расслоение Фано $\pi: V \rightarrow \mathbb{P}^{1}$ удовлетворяет $K^{2}$-условию, если

$$
K_{V}^{2} \notin \operatorname{Int} A_{+}^{2} V
$$

Пусть Ріс $V=\mathbb{Z} K_{V} \oplus \mathbb{Z} F$, где $F$ - класс слоя проекции $\pi$.

ПРЕДЛОЖЕНИЕ 1.2. Если расслоение $\pi: V \rightarrow \mathbb{P}^{1}$ удовлетворяет $K^{2}$-условию, то оно удовлетворяет и К-условию.

ДокАзАтельство. Самопересечение класса $-n K_{V}+l F$ есть

$$
n^{2} K_{V}^{2}+2 n l\left(-K_{V} \cdot F\right),
$$

причем в скобках стоит антиканоническое сечение слоя, т. е. эффективный цикл коразмерности два. В силу $K^{2}$-условия самопересечение не может быть псевдоэффективным при $l<0$, что и требовалось доказать. 
Важность $K$-условия видна из следующего простого факта.

ПредЛОЖениЕ 1.3. Пусть стандартное расслоение Фано $\pi: V \rightarrow S$ удовлетворяет $K$-условию.

(i) Порог канонического присоединения подвижной линейной системы $\Sigma \subset$ $\left|-n K_{V}+\pi^{*} A\right| \operatorname{ecms} c(\Sigma, V)=n$.

(ii) Если подвижная линейная система $\Sigma$ удовлетворяет равенству $c(\Sigma, V)=0$, то $\Sigma$ есть л-подгем подвижной линейной системы $\Sigma_{S}$ на базе $S$.

(iii) Предположим дополнительно, что многообразие $V$ бирачионалъно сверхжесткое: $c_{\text {virt }}(\Sigma)=c(\Sigma, V)$ для любой подвижной линейной системы $\Sigma$. Тогда любая структура рационально связного расслоения на $V$ согласована c $\pi: V \rightarrow S$, m.е. для любого бирачионального отображения $\chi: V \rightarrow V^{\prime}$, где $\pi^{\prime}: V^{\prime} \rightarrow S^{\prime}$ - рационально связное расслоение, существует рациональное доминантное отображение $\varepsilon: S \rightarrow S^{\prime}$ такое, что коммутативна диаграмма

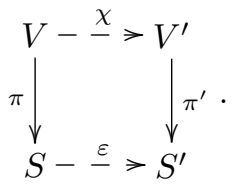

ДоказАТельство. (См. [1; §1].) Утверждение (i) очевидно, поскольку $\pi^{*} A \in A_{+}^{1} V-$ псевдоэффективный класс, в то время как класс $\alpha K_{V}+\pi^{*} A$ при $\alpha>0$ отрицателен на слоях и не может быть псевдоэффективным. Утверждение (ii) следует из (i). Если линейная система $\Sigma^{\prime}$ на многообразии $V^{\prime}$ подвижна и поднята с базы $S^{\prime}$, то ее собственный прообраз $\Sigma=\chi_{*}^{-1} \Sigma^{\prime}$ на $V$ есть подвижная линейная система, удовлетворяющая равенству $c_{\text {virt }}(\Sigma)=c\left(\Sigma^{\prime}, V^{\prime}\right)=0$. В силу предположения о бирациональной сверхжесткости и утверждения (ii) отсюда следует, что $\Sigma$ есть $\pi$-подъем подвижной линейной системы на $S$. Этим доказано утверждение (iii) и завершено доказательство предложения.

Приведем теперь пример нетривиального вычисления порога канонического присоединения на многообразии, не удовлетворяющем $K$-условию.

ПРимеР 1.11. Рассмотрим расслоение Фано $\pi: V \rightarrow \mathbb{P}^{1}$ примера 1.7 (используем обозначения этого примера). Если подвижная линейная система $\Sigma$ есть подсистема полной линейной системы $\left|-n K_{V}+l F\right|$ с $l \in \mathbb{Z}_{+}$, то, как и выше, $c(\Sigma)=n$. Если же $l<0$, то линейная система $\Sigma^{+}=\tau_{*}^{+} \Sigma$ есть подсистема полной линейной системы $\left|-n_{+} K_{V^{+}}+l_{+} F^{+}\right|$на стандартном расслоении Фано $V^{+} / \mathbb{P}^{1}$ из того же семейства. Поскольку теперь $l_{+}=-l \in \mathbb{Z}_{+}$, имеем $c\left(\Sigma^{+}, V^{+}\right)=n_{+}$. Однако $\tau^{+}: V \rightarrow V^{+}$есть бирегулярный изоморфизм в коразмерности единица (т. е. изоморфизм дополнений к замкнутым подмножествам $\Delta \subset V, \Delta^{+} \subset V^{+}$коразмерности два). Поэтому $c(\Sigma, V)=c\left(\Sigma^{+}, V^{+}\right)$, т. е. для $l<0$ имеем

$$
c(\Sigma, V)=n_{+}=n+l .
$$

Другой аналогичный пример дают расслоения Фано $V / \mathbb{P}^{1}$ с нетривиальной (не согласованной со структурой расслоения $\pi: V \rightarrow \mathbb{P}^{1}$ ) бирациональной инволюцией $\tau \in \operatorname{Bir} V$ [31; п. 3.1] и [32; п. 2.3]; в последнем случае это многообразия, описанные в части (iii) теоремы 1.5 ниже. Вычисления в этих примерах 
полностью аналогичны рассмотренным выше: если $\Sigma \subset\left|-n K_{V}+l F\right|$ с $l<0-$ подвижная линейная система, то применение инволюции $\tau$ (которая бирегулярна в коразмерности единица) преобразует $\Sigma$ в систему $\Sigma^{+} \subset\left|-n_{+} K_{V}+l_{+} F\right|$ с $l_{+} \in \mathbb{Z}_{+}$, что позволяет вычислить порог $c(\Sigma, V)=c\left(\Sigma^{+}, V\right)=n_{+}$. Детали см. в указанных работах.

На сегодняшний день имеются два основных подхода к доказательству бирациональной жесткости расслоений Фано: квадратичный и линейный. Квадратичный метод ориентирован на изучение самопересечения $Z=\left(D_{1} \circ D_{2}\right)$ подвижной линейной системы $\Sigma \ni D_{i}$ и непосредственно примыкает к технике доказательства бирациональной жесткости примитивных многообразий Фано, описанной в [1]. Почти все результаты о бирациональной геометрии стандартных расслоений Фано $V / \mathbb{P}^{1}$ получены квадратичным методом.

Линейный метод направлен на изучение особенностей произвольного дивизоpa $D \in \Sigma$ или его ограничения $\left.D\right|_{R}$ на специально подобранное алгебраическое подмногообразие $R \subset V$. Этим методом доказана теорема о прямых произведениях Фано [33], он использован в доказательствах теорем о бирациональной жесткости расслоений $V / \mathbb{P}^{1}$ для упрощения некоторых звеньев [7], [34], а также в доказательстве теоремы о бирациональной геометрии двойных пространств Фано индекса два [35]. Квадратичная техника подробно рассматривается в главе 2 , линейная - в главе 3 .

3.2. Квадратичный метод: основные результаты. Рассмотрим стандартное расслоение Фано $\pi: V \rightarrow \mathbb{P}^{1}$, т. е. Pic $V=\mathbb{Z} K_{V} \oplus \mathbb{Z} F$, где $F$ - класс слоя проекции $\pi$. Предположим дополнительно, что выполнено условие

$$
A^{2} V=\mathbb{Z} K_{V}^{2} \oplus \mathbb{Z} H_{F},
$$

где $H_{F}=\left(-K_{V} \cdot F\right)-$ класс антиканонического сечения слоя. $K^{2}$-условие может быть ослаблено следующим образом.

ОПРЕДЕлЕниЕ 1.6. Говорят, что стандартное расслоение Фано $V / \mathbb{P}^{1}$ удовлетворяет $K^{2}$-условию глубины $\varepsilon \geqslant 0$, если

$$
K_{V}^{2}-\varepsilon H_{F} \notin \operatorname{Int} A_{+}^{2} V .
$$

Иногда для большей ясности $K^{2}$-условие глубины $\varepsilon=0$ будем называть сильным $K^{2}$-условием.

Легко убедиться, что $K$-условие для рассматриваемого класса расслоений Фано принимает вид

$$
-K_{V} \notin \operatorname{Int} A_{\mathrm{mov}}^{1} V \text {. }
$$

Кроме того, очевидно, что если $\varepsilon_{1} \leqslant \varepsilon_{2}$, то $K^{2}$-условие глубины $\varepsilon_{1}$ влечет $K^{2}$-условие глубины $\varepsilon_{2}$.

Расслоения на полные пересечения Фано. Этот класс многообразий соответствует базе $S=\mathbb{P}^{1}$ в примере 1.1. Пусть $a_{*}=\left\{0=a_{0} \leqslant a_{1} \leqslant \cdots \leqslant\right.$ $\left.a_{M+k}\right\}$ - неубывающая последовательность неотрицательных целых чисел, $\mathscr{E}=$ $M+k$

$\bigoplus \mathscr{O}_{\mathbb{P}^{1}}\left(a_{i}\right)$ - локально свободный пучок на $\mathbb{P}^{1}, X=\mathbb{P}(\mathscr{E})$ - соответствующее 
проективное расслоение в смысле Гротендика. Очевидным образом имеем:

$$
\operatorname{Pic} X=\mathbb{Z} L_{X} \oplus \mathbb{Z} R, \quad K_{X}=-(M+k+1) L_{X}+\left(a_{X}-2\right) R,
$$

где $L_{X}-$ класс тавтологического пучка, $R-$ класс слоя морфизма $\pi: X \rightarrow \mathbb{P}^{1}$, $a_{X}=a_{1}+\cdots+a_{M+k}$. Далее, имеем $L_{X}^{M+k+1}=a_{X}$.

Для некоторого набора $k$ чисел $\left(b_{1}, \ldots, b_{k}\right) \in \mathbb{Z}_{+}^{k}$ пусть $G_{i} \in\left|d_{i} L_{X}+b_{i} R\right|-$ такие неприводимые дивизоры, что полное пересечение

$$
V=G_{1} \cap \cdots \cap G_{k} \subset X
$$

есть гладкое подмногообразие. Проекция $\left.\pi\right|_{V}: V \rightarrow \mathbb{P}^{1}$ обозначается тем же самым символом $\pi$, слой $\pi^{-1}(t) \subset V$ - символом $F_{t}$, ограничение $\left.L_{X}\right|_{V}-$ символом $L$.

Расслоение $V / \mathbb{P}^{1}$ есть стандартное расслоение Фано, удовлетворяющее условию (7). Очевидно, что $K_{V}=-L+\left(a_{X}+b_{X}-2\right) F$, где $b_{X}=b_{1}+\cdots+b_{k}$. Легко проверяются формулы

$$
\left(L^{M} \cdot F\right)=\left(H_{F} \cdot L^{M-1}\right)=d, \quad L^{M+1}=d\left(a_{X}+\sum_{i=1}^{k} \frac{b_{i}}{d_{i}}\right),
$$

где $d=d_{1} \cdots d_{k}-$ степень слоя. Отсюда получаем:

$\left(-K_{V} \cdot L^{M}\right)=d\left(2-\sum_{i=1}^{k} \frac{d_{i}-1}{d_{i}} b_{i}\right), \quad\left(K_{V}^{2} \cdot L^{M-1}\right)=d\left(4-a_{X}-\sum_{i=1}^{k} \frac{2 d_{i}-1}{d_{i}} b_{i}\right)$.

Поскольку линейная система $|L|$ свободна, из этих формул сразу вытекают следующие утверждения.

ПРЕДЛОЖЕНИЕ 1.4. (i) Если $a_{X}+\sum_{i=1}^{k} \frac{2 d_{i}-1}{d_{i}} b_{i} \geqslant 4$, то выполнено силъное $K^{2}$-условие.

(ii) Если $a_{X}+\sum_{i=1}^{k} \frac{2 d_{i}-1}{d_{i}} b_{i} \geqslant 2$, то выполнено $K^{2}$-условие глубины 2 .

(iii) Если $\sum_{i=1}^{k} \frac{d_{i}-1}{d_{i}} b_{i} \geqslant 2$, то $-K_{V} \notin \operatorname{Int} A_{+}^{1} V$ и поэтому $-K_{V} \notin \operatorname{Int} A_{\text {mov }}^{1} V$; если, более того, неравенство выше является строгим, то $-K_{V} \notin A_{+}^{1} V$.

Сформулируем теперь основной результат.

Предположим, что многообразие $V$ достаточно общее в своем семействе.

Теорема 1.5 [32]. (i) Многообразие $V$ является бирационально жестким, проекция $\pi: V \rightarrow \mathbb{P}^{1}$ является единственной структурой рационально связного расслоения на $V$ и группы бирачиональных и бирегулярных автоморфизмов совпадают, $\operatorname{Bir} V=\operatorname{Aut} V$, если для целочисленных параметров многообразия $V$ имеет место один из следующих шести случаев:

1) $a_{X}+b_{V} \geqslant 4$

2) $a_{X}=1, b_{V}=2$, 
3) $a_{X}=0, b_{V}=3$,

4) $a_{X}=3, b_{V}=0$,

5) $a_{X}=2, b_{V}=1$,

6) $\left(a_{*}\right)=(0, \ldots, 0,2) u b_{V}=0$.

(ii) При $\left(a_{*}\right)=(0, \ldots, 0,1,1)$ и $b_{V}=0$ общее многообразие $V$ является бирационально сверхжестким. Однако $K$-условие не выполнено: линейная система $\left|-K_{V}-F\right|$ подвижна и задает рациональное отображение $\varphi: V \rightarrow \mathbb{P}^{1}$, слои которого рачионально связны. На многообразии $V$ имеются в точности две нетривиальные структуры рачионально связного расслоения: мор$\oint и з м ~ \pi: V \rightarrow \mathbb{P}^{1}$ и отображение $\varphi$. Существуют единственное, с точностъю до послойного изоморфизма, расслоение Фано $\pi^{+}: V^{+} \rightarrow \mathbb{P}^{1}$ с теми же самыми параметрами $\left(a_{*}\right)=(0, \ldots, 0,1,1)$ u $b_{V^{+}}=0$ и бирациональныи изоморфизм $\chi: V \rightarrow V^{+}$, бирегулярный в коразмерности единица, такие, что коммутативна следующая диаграмма:

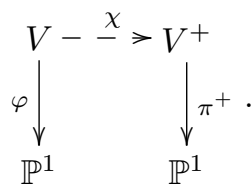

Соответствие $V \rightarrow V^{+}$инволютивно на множестве расслоений Фано этого muna, m.e. $\left(V^{+}\right)^{+}=V$.

(iii) При $a_{X}=0, b_{e}=2$ для некоторого $e \in\{1, \ldots, k\}$ u $b_{i}=0$ для $i \neq$ е многообразие $V$ является бирационально сверхжестким. Однако $K$-условие не выполнено: линейная система $\left|-d_{e} K_{V}-F\right|$ есть пучок рационально связных многообразий. Группа бирациональных автоморфизмов $\operatorname{Bir} V$ нетривиальна и для общего многообразия $V$ порождена бирациональной инволюиией $\tau$, бирегулярной в коразмерности единица, причем $\tau_{*}|F|=\left|-d_{e} K_{V}-F\right|$. На многообразии $V$ имеются в точности две нетривиальные структуры рачионально связного расслоения: проекция $\pi: V \rightarrow \mathbb{P}^{1}$ и отображение $\pi \tau: V \rightarrow \mathbb{P}^{1}$.

Расслоения на циклические накрытия Фано. Пусть $a_{*}=\left\{0=a_{0} \leqslant\right.$ $\left.a_{1} \leqslant \cdots \leqslant a_{M+1}\right\}-$ неубывающая последовательность неотрицательных чисел, $\mathscr{E}=\bigoplus_{i=0}^{M+1} \mathscr{O}_{\mathbb{P}^{1}}\left(a_{i}\right)$ - локально свободный пучок на $\mathbb{P}^{1}, X=\mathbb{P}(\mathscr{E})$ - соответствующее проективное расслоение в смысле Гротендика. Очевидно, имеем

$$
\operatorname{Pic} X=\mathbb{Z} L_{X} \oplus \mathbb{Z} R, \quad K_{X}=-(M+2) L_{X}+\left(a_{X}-2\right) R,
$$

где $L_{X}-$ класс тавтологического пучка, $R-$ класс слоя морфизма $\pi_{X}: X \rightarrow \mathbb{P}^{1}$, $a_{X}=a_{1}+\cdots+a_{M+1}, L_{X}^{M+2}=a_{X}$. Для некоторых $a_{Q}, a_{W} \in \mathbb{Z}_{+}$пусть

$$
Q \sim m L_{X}+a_{Q} R \quad \text { и } \quad W_{X} \sim K\left(l L_{X}+a_{W} R\right)
$$

- дивизоры на $X$, где $Q \subset X$ - гладкое подмногообразие, $W=W_{X} \cap Q$ - гладкий дивизор на $Q$. Пусть

$$
\sigma: V \rightarrow Q
$$


- $K$-листное циклическое накрытие многообразия $Q$, разветвленное над дивизором $W$. Проекцию $\left.\pi_{X}\right|_{Q}$ обозначим символом $\pi_{Q}$, проекцию $\pi_{Q} \circ \sigma: V \rightarrow \mathbb{P}^{1}-$ символом $\pi$. Слой $\pi_{Q}^{-1}(t), t \in \mathbb{P}^{1}$, обозначаем символом $G_{t}$ (или просто $G$, когда ясно, о какой точке $t \in \mathbb{P}^{1}$ идет речь), слой $\pi^{-1}(t) \subset V-$ символом $F_{t}$ или $F$. Пусть $L_{Q}=\left.L_{X}\right|_{Q}$ и $L=\sigma^{*} L_{Q}$. Очевидно, что

$$
\operatorname{Pic} V=\mathbb{Z} L \oplus \mathbb{Z} F, \quad K_{V}=-L+\left(a_{X}+a_{Q}+(K-1) a_{W}-2\right) F .
$$

Легко проверяются формулы $\left(L^{M} \cdot F\right)=m K, L^{M+1}=K\left(m a_{X}+a_{Q}\right)$. Отсюда получаем $\left(-K_{V} \cdot L^{M}\right)=K\left((1-m) a_{Q}-m(K-1) a_{W}+2 m\right)$ и

$$
\left(K_{V}^{2} \cdot L^{M-1}\right)=K\left(-m a_{X}+(1-2 m) a_{Q}-2 m(K-1) a_{W}+4 m\right) .
$$

Для удобства записываем параметры накрытия $V$ в виде

$$
\left(\left(a_{1}, \ldots, a_{M+1}\right),\left(a_{Q}, a_{W}\right)\right),
$$

причем среди чисел $a_{1}, \ldots, a_{M+1}$ указываем только ненулевые, если таковые имеются, иначе пишем просто (0). Удобство этих обозначений связано с тем, что индивидуального анализа требуют лишь накрытия $V$, у которых почти все параметры равны нулю. В самом деле, из выписанных выше явных формул немедленно вытекают следующие утверждения.

ПредЛОЖЕНИЕ 1.5. (i) Многообразие $V$ удовлетворяет силъному $K^{2}$-условию, т.е. $K^{2}$-условию глубины 0, если имеет место один из следующих случаев:

1) $a_{W} \geqslant 1$

2) $a_{W}=0, a_{Q} \geqslant 3$,

3) $a_{W}=0, a_{Q}=2, a_{X} \geqslant 1$,

4) $a_{W}=0, a_{Q}=1, a_{X} \geqslant 3$,

5) $a_{W}=a_{Q}=0, a_{X} \geqslant 4$.

(ii) Если $a_{W}=0, a_{Q}=2, a_{X}=0$, то многообразие $V$ удовлетворяет $K^{2}$-условию глубины $2 / \mathrm{m}$.

(iii) Если $a_{W}=0, a_{Q}=1$, то многообразие $V$ удовлетворяет $K^{2}$-условию глубины $1 / m$ при $a_{X}=2$ и глубины $(1+1 / m)$ при $a_{X}=1$.

(iv) Eсли $a_{W}=a_{Q}=0$, то многообразие $V$ удовлетворяет $K^{2}$-условию глубины 1 при $a_{X}=3$ и глубины 2 при $a_{X}=2$.

ДокАЗАТЕльство. Предложение немедленно следует из того, что для любого неприводимого подмногообразия $Y$ имеет место неравенство $\left(Y \cdot L^{\operatorname{dim} Y}\right) \geqslant 0$.

Сформулируем теперь основной результат.

Предполагаем, что циклическое накрытие $V$ достаточно общее в построенном семействе.

Теорема 1.6 [8]. (i) Многообразие $V$ является бирационально сверхжестким, проекция $\pi: V \rightarrow \mathbb{P}^{1}$ является единственной структурой рационально связного расслоения на $V$ и группы бирачионалъных и бирегулярных автоморфизмов многообразия $V$ совпадают, $\operatorname{Bir} V=\operatorname{Aut} V$, если иелочисленные параметры многообразия $V$ либо удовлетворяют любому из условий 1)-5) предложения 1.5 (i), либо образуют один из следуюших шести наборов: $((2),(0,0))$, $((2),(1,0)),((1,1),(1,0)),((3),(0,0)),((1,2),(0,0)),((1,1,1),(0,0))$. 
(ii) Многообразие $V$ типа $((1,1),(0,0))$ является бирачионально сверхжестким. Однако $K$-условие не выполнено: линейная система $\left|-K_{V}-F\right|$ подвижна и задает бирациональное отображение $\varphi: V \rightarrow \mathbb{P}^{1}$, слои которого рационально связны. На многообразии $V$ имеются в точности две структуры рационально связного расслоения: проекиия $\pi$ и отображение $\varphi$. Существуют единственное ( $с$ точностью до послойного изоморфизма) расслоение на чиклические накрытия Фано $\pi^{+}: V^{+} \rightarrow \mathbb{P}^{1}$ того же типа $((1,1),(0,0))$ и бирачиональный изоморфизм $\chi: V \rightarrow V^{+}$, бирегулярньй в коразмерности единиц, такие, что коммутативна диаграмма отображений

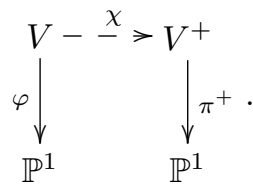

Соответствие $V \rightarrow V^{+}$инволютивно, т.е. $\left(V^{+}\right)^{+}=V$.

(iii) Многообразие $V$ типа $((0),(2,0))$ является бирационально сверхжестким. Однако $K$-условие не выполнено: линейная система $\left|-m K_{V}-F\right|$ подвижна и задает бирациональное отображение, слои которого рационально связны. Группа бирациональных автоморфизмов Bir $V$ строго больше группы бирегулярных автоморбизмов: она содержит нетривиальную бирациональную инволюиию $\tau \in \operatorname{Bir} V \backslash$ Aut $V$, причем $\operatorname{Bir} V \cong(\mathbb{Z} / 2 \mathbb{Z}) \times(\mathbb{Z} / K \mathbb{Z})$, где $\mathbb{Z} / 2 \mathbb{Z}=\{$ id, $\tau\}$. На $V$ имеются ровно две структуры рационально связного расслоения: проекция $\pi$ и рациональное отображение $\pi \tau: V \rightarrow \mathbb{P}^{1}$, причем $\left|-m K_{V}-F\right|=\tau_{*}|F|$.

Отметим очевидный параллелизм теорем 1.5 и 1.6 (их доказательства, однако, существенно отличаются). Бирациональная (сверх)жесткость доказана и для некоторых других семейств расслоений Фано над $\mathbb{P}^{1}$, см. [3], [36]-[38]. В теоремах 1.5 и 1.6 описаны наиболее "массовые" семейства.

Многообразия с пучком поверхностей дель Пеццо. Этот класс трехмерных рационально связных многообразий долгое время не поддавался изучению. Бирациональная жесткость подавляющего большинства этих многообразий доказана в [3]. За этой работой последовали [26], [27], где бирациональная геометрия почти всех оставшихся типов многообразий с пучком поверхностей дель Пеццо степени 1 и 2 была полностью описана. Эта работа подытожена в обзоре [4].

Пусть $V \stackrel{\pi}{\rightarrow} \mathbb{P}^{1}$ - расслоение на поверхности дель Пеццо степени $d \in\{1,2,3\}$, $\operatorname{Pic} V=\mathbb{Z} K_{V} \oplus \mathbb{Z} F$, многообразие $V$ гладкое, а при $d=3$ достаточно общее.

ТеОрема 1.7 [3]. Предположим, что расслоение $V / \mathbb{P}^{1}$ удовлетворяет $K^{2}$-условию: $K_{V}^{2} \notin \operatorname{Int} A_{+}^{2} V$. Тогда многообразие $V$ бирационально жесткое (сверхжесткое при $d=1)$, проекция $\pi: V \rightarrow \mathbb{P}^{1}$ есть единственная структура рационально связного расслоения на многообразии $V$, а факторгруппа группы бирачиональных автоморфизмов по нормальной подгруппе бирачиональных автоморфизмов общего слоя $\operatorname{Bir} F_{\eta}$ конечна, в общем случае тривиальна. 
При $d=1$ группа Bir $F_{\eta}$ совпадает с Aut $F_{\eta}$ и конечна, при $d=2$ она порождается подгруппой Aut $F_{\eta}$ и инволюциями, связанными с сечениями расслоения $\pi$, при $d=3$ она порождается подгруппой Aut $F_{\eta}$ и инволюциями, связанными с сечениями и бисечениями проекции $\pi$, описанными в примере 1.4 (исходное описание этих групп см. в [5], [39]).

Технике доказательств теорем 1.5, 1.6 и 1.7 посвящена глава 2.

3.3. Линейный метод: основные результаты. В основе линейного метода лежит доказанная в [40] в размерности 3 и в [41] в произвольной размерности теорема об "обращении присоединения". Сформулируем частный случай этого результата, используемый в теории бирациональной жесткости. Обсуждение и доказательство обращения присоединения приведены в 22 главы 3.

Теорема 1.8 (обращение присоединения). Пусть $x \in X-$ росток $\mathbb{Q}$-факториального терминального многообразия, $D$ - эббективный $\mathbb{Q}$-дивизор, носитель которого содержит точку $x$. Пусть $R \subset X$ - неприводимое подмногообразие коразмерности единица, $R \not \subset \operatorname{Supp} D$, причем $R$ - дивизор Картье. Предположим, что пара $(X, D)$ не канонична в точке $x$, но канонична вне этой точки, т.е. точка $x$ - изолированный центр не канонических особенностей этой парь. Тогда пара $\left(R, D_{R}=\left.D\right|_{R}\right)$ не логканонична в точке $x$.

Обращение присоединения (сформулированное выше не в самом общем варианте, а лишь в таком, который будет реально нужен) используется для исключения максимальных особенностей подвижных линейных систем $\Sigma$ на изучаемом рационально связном многообразии $V$ следующим образом. Пусть $R \subset V$ - неприводимый приведенный дивизор Картье (как правило, многообразие $V$ гладкое или имеет простейшие особенности, так что предположения теоремы 1.8 выполнены автоматически), $D \in \Sigma$ - общий дивизор. В силу подвижности системы $\Sigma$ имеем $R \not \subset \operatorname{Supp} D$, так что ограничение $D_{R}$ корректно определено. Далее, если пара $\left(R, n^{-1} D_{R}\right)$ логканонична в точке $x \in R$, то пара $\left(V, n^{-1} \Sigma\right)$ канонична в точке $x$, т. е. не существует максимальных особенностей системы $\Sigma$, центр которых есть точка $x$. Описанная процедура сводит изучение бирациональной геометрии многообразия $V$ к изучению геометрии дивизора $R$, что иногда существенно упрощает работу. Отметим, что эту процедуру можно повторить (если только выполнены предположения теоремы 1.8), сводя изучение особенностей пары $\left(V, n^{-1} \Sigma\right)$ к изучению особенностей пары $\left(R, n^{-1} D_{R}\right)$, где $R \subset V$ - неприводимое подмногообразие (не обязательно дивизор). Наиболее важный случай - это ограничение на слой $R=\pi^{-1}(s)$ расслоения $\pi: V \rightarrow S$.

Имеются три группы результатов, существенно использующих линейный метод.

Прямые произведения Фано. Теоремы о бирациональной геометрии прямых произведений Фано образуют самую большую (на сегодняшний день) группу результатов [32]-[34], [42], [43].

Пусть $F$ - многообразие Фано размерности $\geqslant 3$ с $\mathbb{Q}$-факториальными терминальными особенностями и числом Пикара $\rho(F)$, равным 1. 
ОПРЕДЕЛЕНиЕ 1.7. Скажем, что многообразие $F$ удовлетворяет условию дивизориальной каноничности или условию $(\mathrm{C})$ (соответственно условию дивизориалъной логканоничности или условию (L)), если для любого эффективного дивизора $D \in\left|-n K_{F}\right|, n \geqslant 1$, пара

$$
\left(F, \frac{1}{n} D\right)
$$

имеет канонические (соответственно логканонические) особенности. Если пара (8) имеет канонические особенности для общего дивизора $D \in \Sigma \subset\left|-n K_{F}\right|$ любой подвижной линейной системы, то будем говорить, что $F$ удовлетворяет условию подвижной каноничности или условию (M).

В явном виде, условие (C) формулируется следующим образом: для любого бирационального морфизма $\varphi: \widetilde{F} \rightarrow F$ и исключительного дивизора $E \subset \widetilde{F}$ имеет место неравенство

$$
\nu_{E}(D) \leqslant n a(E) .
$$

Неравенство (9) противоположно неравенству Нётера-Фано. Условие (L) слабее: требуется выполнение неравенства

$$
\nu_{E}(D) \leqslant n(a(E)+1) .
$$

Напомним, что в $(9)$ и $(10)$ число $a(E)$ - дискрепантность исключительного дивизора $E \subset \widetilde{F}$ относительно модели $F$. Неравенство (10) противоположно логнеравенству Нётера-Фано. Условие (М) означает, что (9) имеет место для общего дивизора $D$ любой подвижной системы $\Sigma \subset\left|-n K_{F}\right|$ и любого дискретного нормирования $\nu_{E}$.

В другой терминологии, условие (L) означает, что глобальный логканонический порог многообразия $F$ равен единице: $\operatorname{lct}(F)=1$. Условие $(\mathrm{C})$ означает, что глобалъный канонический порог равен единице $\mathrm{ct}(F)=1$. Значение этих условий раскрывается следующим фактом [33].

Теорема 1.9. Пусть примитивные многообразия Фано $F_{1}, \ldots, F_{K}$, где $K \geqslant 2$, удовлетворяют условиям (L) и (M). Тогда их прямое произведение

$$
V=F_{1} \times \cdots \times F_{K}
$$

- бирачионально сверхжесткое многообразие, т.е. для любой подвижной линейной системы $\Sigma$ ее виртуалъный и реальный пороги канонического присоединения совпадают:

$$
c(\Sigma)=c_{\mathrm{virt}}(\Sigma)
$$

В частности, верны следующие утверждения.

(i) Все структуры рационально связного расслоения на $V$ суть проекции на прямые сомножители. Точнее, пусть $\beta: V^{\sharp} \rightarrow S^{\sharp}-$ рационально связное расслоение и $\chi: V \rightarrow V^{\sharp}-$ бирациональное отображение. Тогда существуют множество индексов $I=\left\{i_{1}, \ldots, i_{k}\right\} \subset\{1, \ldots, K\}$ и бирачиональное отображение

$$
\alpha: F_{I}=\prod_{i \in I} F_{i} \rightarrow S^{\sharp}
$$


такие, что коммутативна диаграмма

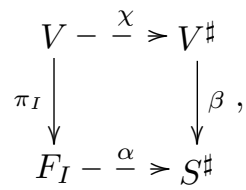

m.е. $\beta \circ \chi=\alpha \circ \pi_{I}$, где $\pi_{I}: \prod_{i=1}^{K} F_{i} \rightarrow \prod_{i \in I} F_{i}$ - естественная проекция на прямой сомножитель. $B$ частности, на многообразии $V$ нет структур расслоения на рационально связные многообразия размерности строго менъшей $\min \left\{\operatorname{dim} F_{i}\right\} ;$ тем самым $V$ не имеет структур расслоения на коники и рациональные поверхности.

(ii) Группа бирационалъных автоморфизмов и группа бирегулярных автоморфизмов многообразия $V$ совпадают:

$$
\operatorname{Bir} V=\operatorname{Aut} V \text {. }
$$

(iii) Многообразие $V$ нерационально.

Теорема 1.9 доказана в [33] для гладких примитивных многообразий Фано, однако доказательство проходит дословно в более общем случае, описанном выше. Очевидно, что условие (C) сильнее, чем каждое из условий $(\mathrm{L})$ и $(\mathrm{M})$. Для применения теоремы 1.9 достаточно проверить, что многообразие Фано из заданного семейства удовлетворяет условию $(\mathrm{C})$ (или обоим условиям (L) и $(\mathrm{M}))$.

Для общих гиперповерхностей Фано $F_{d} \subset \mathbb{P}^{d}, d \geqslant 6$, и общих двойных пространств индекса единица $F_{2} \rightarrow \mathbb{P}^{d}, d \geqslant 3$, выполнение условия (C) доказано в [33]. Для общих двойных гиперповерхностей Фано $F \rightarrow Q_{m} \subset \mathbb{P}^{d}, d \geqslant 7$, разветвленных над $W=W_{2 l}^{*} \cap Q_{m}$, где $Q_{m}$ и $W_{2 l}^{*}$ - общие гиперповерхности степеней $m$ и $2 l$, соответственно, $m+l=d$, условие $(\mathrm{C})$ доказано в [34]. Для общих взвешенных гиперповерхностей Фано размерности три условие (L) проверено в [42].

Доказательство теоремы 1.9 и дивизориальной каноничности гиперповерхностей Фано $F_{d} \subset \mathbb{P}^{d}$ приведено в $\S 1$ главы 3 .

Расслоения Фано над $\mathbb{P}^{1}$. Применение линейного метода позволяет упростить доказательство бирациональной жесткости расслоений $V / \mathbb{P}^{1}$ [34], [43]. Предположим, что $\Sigma \subset\left|-n K_{V}+l F\right|$ - подвижная линейная система, где $l \in \mathbb{Z}_{+}$, а слой $F^{*}=\pi^{-1}\left(t_{*}\right)$ проекции $\pi: V \rightarrow \mathbb{P}^{1}$ удовлетворяет условию $(\mathrm{L})$. Тогда в силу обращения присоединения центр любой максимальной особенности системы $\Sigma$ не может содержаться в слое $F^{*}$ : он либо накрывает базу $\mathbb{P}^{1}$, либо содержится в другом слое. В частности, если все слои удовлетворяют условиям (L) и $(\mathrm{M})$, то система $\Sigma$ вообще не имеет максимальных особенностей, откуда следует, что

$$
c_{\text {virt }}(\Sigma)=c(\Sigma, V)=n .
$$

Рассуждения такого типа могут быть использованы и для исключения отдельных типов максимальных особенностей [7]. 
Многообразия Фано индекса два. Этот результат выходит за пределы настоящего обзора, потому что относится к нежестким многообразиям (на самом деле, является первым полноценным примером полного описания бирациональной геометрии нежестких многообразий произвольной размерности). Однако он непосредственно примыкает к предыдущим результатам и существенно использует линейный метод. Поэтому мы сформулируем его ниже и кратко обсудим схему его доказательства в 33 главы 3.

Пусть $M \geqslant 5$ и $W=W_{2(M-1)} \subset \mathbb{P}^{M}$ - гладкая гиперповерхность степени $2(M-1)$. Рассмотрим двойное накрытие

$$
\sigma: V \rightarrow \mathbb{P}^{M}
$$

разветвленное над $W$. Многообразие $V$ есть многообразие Фано индекса два: $\operatorname{Pic} V=\mathbb{Z} H$, где $H$ - обильная образующая, $K_{V}=-2 H$, класс $H$ есть $\sigma$-подъем гиперплоскости в $\mathbb{P}$. На многообразии $V$ имеются следующие естественные структуры рационально связного расслоения: пусть $\alpha_{P}: \mathbb{P} \rightarrow \mathbb{P}^{1}-$ линейная проекция из произвольного линейного подпространства $P$ коразмерности два, тогда отображение

$$
\pi_{P}=\alpha_{P} \circ \sigma: V \rightarrow \mathbb{P}^{1}
$$

расслаивает $V$ на $(M-1)$-мерные многообразия Фано индекса 1. Предполагаем, что многообразие $V$ достаточно общее.

Tеорема 1.10 [35]. Пусть $M \geqslant 5$ u $\chi: V \rightarrow Y-$ бирациональное отображение на пространство ращионально связного расслоения $\lambda: Y \rightarrow S$. Тогда $S=\mathbb{P}^{1}$ и для некоторого изоморфизма $\beta: \mathbb{P}^{1} \rightarrow S$ и некоторого подпространства $P \subset \mathbb{P}$ коразмерности два имеем $\lambda \circ \chi=\beta \circ \pi_{P}$, т.е. коммутативна диаграмма

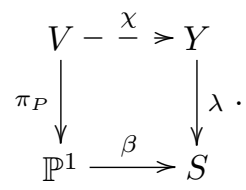

СлЕДСтвиЕ 1.4. (i) На многообразии $V$ нет структур рационально связного расслоения с базой размерности $\geqslant 2$. В частности, на $V$ нет структур расслоения на коники и поверхности дель Пеццо, а само многообразие $V$ нерационально.

(ii) Предположим, что имеется бирациональное отображение $\chi: V \rightarrow Y$, где $Y$ - многообразие Фано индекса $r \geqslant 2$ с факториальными терминальными особенностями такое, что

$$
\operatorname{Pic} Y=\mathbb{Z} H_{Y}, \quad \text { əде } K_{Y}=-r H_{Y},
$$

причем линейная система $\left|H_{Y}\right|$ непуста и свободна. Тогда $r=2$ и отображение ұ есть бирегулярный изоморфизм.

(iii) Группа бирачиональных автоморфизмов и группа бирегулярных автоморфизмов многообразия $V$ совпадают:

$$
\operatorname{Bir} V=\text { Aut } V=\mathbb{Z} / 2 \mathbb{Z} \text {. }
$$


ДокАЗАТЕЛЬСтво СЛЕДСТВИЯ 1.4. Утверждение (i) и равенство $r=2$ в (ii) очевидны (любая линейная подсистема проективной размерности $\leqslant r-1$ в полной линейной системе $\left|H_{Y}\right|$ определяет структуру рационально связного расслоения на $Y$ ). Далее, $\chi$-прообраз общего дивизора в системе $\left|H_{Y}\right|$ есть дивизор в линейной системе $|H|$ по теореме 1.10 , что завершает доказательство утверждения (ii). Часть (iii) очевидным образом следует из (ii). Доказательство закончено.

\section{Глава 2}

\section{Расслоения Фано над проективной прямой}

\section{§ 1. Достаточные условия бирациональной жесткости}

\section{1. Формулировка достаточных условий. Начало доказательства.} Пусть $\pi: V \rightarrow \mathbb{P}^{1}$ - стандартное расслоение Фано, т. е. $V$ - гладкое многообразие, $\operatorname{Pic} V=\mathbb{Z} K_{V} \oplus \mathbb{Z} F$, где $F$ - класс слоя проекции $\pi$. Предположим дополнительно, что выполнено условие

$$
A^{2} V=\mathbb{Z} K_{V}^{2} \oplus \mathbb{Z} H_{F},
$$

где $H_{F}=\left(-K_{V} \cdot F\right)$ - обильное антиканоническое сечение слоя, и что каждый слой $F=F_{t}=\pi^{-1}(t), t \in \mathbb{P}^{1}$, есть многообразие Фано, имеющее самое большее, невырожденные квадратичные особенности, причем

$$
A^{1} F=\operatorname{Pic} F=\mathbb{Z} K_{F} \quad \text { и } \quad A^{2} F=\mathbb{Z} H_{F}^{2},
$$

где $K_{F}=-H_{F}$ и $H_{F}$ рассматривается как элемент группы $A^{1} F$. Эти условия выполнены для почти всех семейств стандартных расслоений Фано, успешно изученных к настоящему времени. Исключение составляют расслоения со слоем размерности 2, 3 и 4, где малая размерность позволяет привлекать дополнительные соображения. Крайний случай - расслоения на кубические поверхности - рассмотрен ниже в $\S 3$.

Сформулируем теперь дополнительные условия, из которых выводится бирациональная (сверх)жесткость расслоения Фано. Эти условия следует понимать как некоторые условия общности положения для расслоения $V / \mathbb{P}^{1}$; точнее, общие (в своем семействе) расслоения $V / \mathbb{P}^{1}$ удовлетворяют этим условиям. Под степенъю неприводимого подмногообразия $Y \subset V$, накрывающего базу $\mathbb{P}^{1}$, $\pi(Y)=\mathbb{P}^{1}$ (такие подмногообразия называются горизонтальными), понимается число

$$
\operatorname{deg} Y=\left(Y \cdot F \cdot\left(-K_{V}\right)^{\operatorname{dim} Y-1}\right) .
$$

ОПРЕДЕЛЕНИЕ 2.1. Расслоение $V / \mathbb{P}^{1}$ удовлетворяет

- условию $(v)$, если для любого неприводимого вертикального подмногообразия $Y$ коразмерности два (т. е. $Y \subset F_{t}$ - простой дивизор, $\left.t=\pi(Y)\right)$ и любой гладкой точки $o \in F_{t}$ выполнено неравенство

$$
\frac{\operatorname{mult}_{o} Y}{\operatorname{deg} Y} \leqslant \frac{2}{\operatorname{deg} V} ;
$$


- условию $(f)$, если для любого неприводимого вертикального подмногообразия $Y$ коразмерности три (т. е. $\operatorname{codim}_{F} Y=2, F=F_{t} \supset Y$ ) и любой гладкой точки слоя $o \in F$ выполнено неравенство

$$
\frac{\operatorname{mult}_{o} Y}{\operatorname{deg} Y} \leqslant \frac{4}{\operatorname{deg} V} ;
$$

- условию $(v s)$, если для любого вертикального подмногообразия $Y \subset F_{t}$ коразмерности 2 (относительно $V$, т. е. простого дивизора на $F_{t}$ ), особой точки $o \in F_{t}$ и бесконечно близкой точки $x \in \widetilde{F}_{t}$, где $\varphi: \widetilde{F}_{t} \rightarrow F_{t}$ - раздутие точки $o$, $\varphi(x)=o, \widetilde{Y} \subset \widetilde{F}_{t}-$ собственный прообраз подмногообразия $Y$ на $\widetilde{F}_{t}$, выполнены оценки

$$
\frac{\operatorname{mult}_{o} Y}{\operatorname{deg} Y} \leqslant \frac{4}{\operatorname{deg} V}, \quad \frac{\operatorname{mult}_{x} \tilde{Y}}{\operatorname{deg} Y} \leqslant \frac{2}{\operatorname{deg} V} ;
$$

- условию $(f s)$, если для любого вертикального подмногообразия $Y \subset F_{t}$ коразмерности 3 (относительно $V$, т. е. подмногообразия коразмерности 2 на $F_{t}$ ), особой точки $o \in F_{t}$ и бесконечно близкой точки $x \in \widetilde{F}_{t}$, где $\varphi: \widetilde{F}_{t} \rightarrow F_{t}$ - раздутие точки $o, \varphi(x)=o, \widetilde{Y} \subset \widetilde{F}_{t}-$ собственный прообраз подмногообразия $Y$ на $\widetilde{F}_{t}$, выполнены оценки

$$
\frac{\operatorname{mult}_{o} Y}{\operatorname{deg} Y} \leqslant \frac{6}{\operatorname{deg} V}, \quad \frac{\operatorname{mult}_{x} \tilde{Y}}{\operatorname{deg} Y} \leqslant \frac{3}{\operatorname{deg} V} ;
$$

- условию $(h)$, если для любого горизонтального подмногообразия $Y$ коразмерности 2 и точки $o \in Y$ выполнена оценка

$$
\frac{\operatorname{mult}_{o} Y}{\operatorname{deg} Y} \leqslant \frac{4}{\operatorname{deg} V} .
$$

Как и в предыдущем обзоре [1], для удобства обозначений отношение кратности к степени будем в дальнейшем записывать единым символом:

$$
\frac{\text { mult }_{o}}{\operatorname{deg}} Y=\frac{\operatorname{mult}_{o} Y}{\operatorname{deg} Y} .
$$

Достаточные условия бирациональной (сверх)жесткости, применимые к большинству стандартных расслоений Фано $V / \mathbb{P}^{1}$, собраны в следующем утверждении.

Теорема 2.1. (i) Предположим, что стандартное расслоение Фано $V / \mathbb{P}^{1}$ удовлетворяет (сильному) $K^{2}$-условию и условиям $(v),(v s)$ u $(h)$. Тогда расслоение $V / \mathbb{P}^{1}$ есть бирационально сверхжесткое расслоение Фано.

(ii) Предположим, что стандартное расслоение Фано $V / \mathbb{P}^{1}$ удовлетворяет $K^{2}$-условию глубины 2, условиям $(v)$, (vs) и хотя бы одному из условий $(f)$ или $(f s)$ в любой точке $o \in V$. Тогда для любой подвижной линейной системы $\Sigma \subset\left|-n K_{V}+l F\right|$ c $l \in \mathbb{Z}_{+}$ее виртуальный и фактический пороги канонического присоединения совпадают:

$$
c_{\text {virt }}(\Sigma)=c(\Sigma)=n .
$$

$B$ частности, если $V / \mathbb{P}^{1}$ удовлетворяет $K$-условию, то это расслоение является бирационально сверхжестким. 
(iii) Предположим, что стандартное расслоение Фано $V / \mathbb{P}^{1}$ удовлетворяет $K^{2}$-условию глубины 2 и условиям $(v)$ u $(f)$. Если центр любой максимальной особенности подвижной линейной системы $\Sigma \subset\left|-n K_{V}+l F\right| c l \in \mathbb{Z}_{+}$не есть особая точка слоя, то виртуальный и фактический пороги канонического присоединения совпадают:

$$
c_{\text {virt }}(\Sigma)=c(\Sigma)=n
$$

В частности, если последнее предположение выполнено для любой подвижной линейной системы на $V$, а расслоение $V / \mathbb{P}^{1}$ удовлетворяет $K$-условию, то это расслоение является бирационально сверхжестким.

Утверждение (i) доказано в [36] (см. также [31]). Утверждение (ii) существенно сильнее (предполагается $K^{2}$-условие глубины 2, что позволяет охватить многие расслоения, не удовлетворяющие $K^{2}$-условию) и требует более тонких рассуждений [31]. Утверждение (iii) - это упрощенный вариант (ii): максимальные особенности, лежащие над особыми точками слоев, исключены из рассмотрения (подразумевается, что они исключены другим методом).

Оставшаяся часть настоящего параграфа посвящена доказательству утверждения (iii). Мы следуем [8], [32]. Доказательство утверждения (i) проще, а разбор максимальных особенностей, лежащих над квадратичными точками слоев (единственное, что необходимо добавить к доказательству части (iii), чтобы получить утверждение (ii)), полностью аналогичен приводимым ниже рассуждениям.

Итак, зафиксируем подвижную линейную систему $\Sigma \subset\left|-n K_{V}+l F\right|$ с $l \in \mathbb{Z}_{+}$. Очевидно, что $c(\Sigma)=n$, причем $n=0$ тогда и только тогда, когда $\Sigma$ составлена из пучка слоев $|F|$. Предположим, что имеет место неравенство $c_{\text {virt }}(\Sigma)<n$, в частности, $n \geqslant 1$. Это означает, что существует бирациональный морфизм $\varphi: \widetilde{V} \rightarrow V$ неособых многообразий такой, что $c(\widetilde{\Sigma}, \widetilde{V})<n$, где $\widetilde{\Sigma}-$ собственный прообраз $\Sigma$ на $\widetilde{V}$. Отсюда немедленно вытекает, что пара $\left(V, n^{-1} \Sigma\right)$ неканонична, т. е. система $\Sigma$ имеет максимальную особенность $E \subset \widetilde{V}$. Рассмотрим ее центр $B=\varphi(E) \subset V$, неприводимое подмногообразие коразмерности не меньше двух.

Пусть (см. [1; гл. 2])

$$
\begin{aligned}
\varphi_{i, i-1}: & V_{i} \longrightarrow V_{i-1} \\
& \cup \quad \cup \\
& E_{i} \longrightarrow B_{i-1}
\end{aligned}
$$

- последовательность раздутий с неприводимыми центрами $B_{i-1} \subset V_{i-1}$, однозначно определенная условиями

1) $V_{0}=V, B_{0}=B, i=1, \ldots, N$;

2) $B_{j}=\operatorname{centre}\left(E, V_{j}\right) \subset V_{j}, E_{j+1}=\varphi_{j+1, j}^{-1}\left(B_{j}\right)$;

3) нормирование $\nu_{E_{N}}$ совпадает с $\nu_{E}$.

Иными словами, бирациональное отображение $V_{N} \rightarrow \widetilde{V}$ бирегулярно в общей точке дивизора $E_{N}$ и преобразует $E_{N}$ в $E$. Напомним, что многообразия $V_{1}, \ldots, V_{N}$, вообще говоря, могут иметь особенности, так как раздуваемые 
подмногообразия $B_{j}$ не обязаны быть гладкими. Однако $V_{j}$ неособо в общей точке $B_{j}$. Символом $\Sigma^{j}$ обозначим собственный прообраз линейной системы $\Sigma$ на $V_{j}$. Положим

$$
\nu_{j}=\operatorname{mult}_{B_{j-1}} \Sigma^{j-1}, \quad \delta_{j}=\operatorname{codim} B_{j-1}-1 .
$$

На множестве исключительных дивизоров $\left\{E_{1}, \ldots, E_{N}\right\}$ зададим обычным образом структуру ориентированного графа: $E_{i}$ и $E_{j}$ соединены ориентированным ребром, если и только если $i>j$ и

$$
B_{i-1} \subset E_{j}^{i-1}
$$

обозначение: $i \rightarrow j$. Как обычно, пусть при $i>j$

$$
p_{i j}=\sharp\left\{\text { пути из } E_{i} \text { в } E_{j}\right\} \geqslant 1,
$$

$p_{i i}=1$ по определению. Положим $p_{i}=p_{N i}$. Неравенство Нётера-Фано принимает традиционный вид

$$
\sum_{i=1}^{N} p_{i} \nu_{i}>n \sum_{i=1}^{N} p_{i} \delta_{i} .
$$

ПРЕДЛОЖЕНИЕ 2.1. Центр $B$ максимальной особенности $E$ на $V$ содержится в некотором слое $F_{t}=\pi^{-1}(t), t \in \mathbb{P}^{1}$.

ДокАЗАтельство. Предположим противное: $\pi(B)=\mathbb{P}^{1}$. Пусть $F \subset V$ слой общего положения. Легко видеть, что ограничение $\Sigma_{F}=\left.\Sigma\right|_{F}$ линейной системы $\Sigma$ на $F$ есть подвижная линейная система $\Sigma_{F} \subset\left|n H_{F}\right|$, причем пара $\left(F, n^{-1} \Sigma_{F}\right)$ неканонична, т. е. $\Sigma_{F}$ имеет максимальную особенность. Проще всего определить эту максимальную особенность таким образом: пусть $\widetilde{F} \subset \widetilde{V}-$ собственный прообраз, $E_{F}$ - неприводимая компонента замкнутого множества $\widetilde{F} \cap E$. Иначе говоря, можно ограничить последовательность раздутий (14) на слой $F$. Дискрепантность не меняется: $a\left(E_{F}, F\right)=a(E, V)$. Центр особенности $E_{F}$ есть $\varphi\left(E_{F}\right)$ - неприводимая компонента замкнутого множества $B \cap F$.

По предположению, Pic $F=\mathbb{Z} K_{F}, A^{2} F=\mathbb{Z} K_{F}^{2}$ и для каждого неприводимого подмногообразия $Y \subset F$ коразмерности два в каждой точке выполнено неравенство (13). Из этих предположений вытекает каноничность любой пары $\left(F, n^{-1} \Sigma_{F}\right)$, где $\Sigma_{F} \subset\left|n H_{F}\right|-$ подвижная линейная система (см. [1; гл. 2]). Это противоречие завершает доказательство предложения.

Итак, $B \subset F=F_{t}$ содержится в слое.

Лемма 2.1. Имеет место неравенство $\operatorname{codim}_{F} B \geqslant 2$.

ДокАЗАтЕЛьСтво. Предположим противное: $B \subset F$ - простой дивизор. Пусть $D \in \Sigma$ - общий дивизор, $D_{F}$ - его ограничение на $F$. В силу неравенства Нётера-Фано mult $_{B} D>n$, так что $D_{F}=\alpha B+D^{\sharp}$, где $\alpha>n$ и $D^{\sharp}-$ эффективный дивизор на $F$. Однако $D_{F} \sim n H_{F}$, что сразу дает противоречие. Лемма доказана. 
Пусть $\mathscr{M}=\left\{T_{1}, \ldots, T_{k}\right\}$ - множество всех максимальных особенностей линейной системы $\Sigma$ на $\widetilde{V}$. Как было доказано выше, центр $B_{E}=\operatorname{centre}\left(\nu_{E}\right)$ любой максимальной особенности $E \in \mathscr{M}$ содержится в некотором слое $F_{t}$. Множество $\mathscr{M}$ конечно, так что существует конечное множество точек $t \in \mathbb{P}^{1}$, слои $F_{t}$ над которыми содержат центры максимальных особенностей. Положим $\mathscr{M}_{t}=\left\{E \in \mathscr{M} \mid B_{E} \subset F_{t}\right\}$ и

$$
e(E)=\nu_{E}(\Sigma)-n a(E, V)>0
$$

для $E \in \mathscr{M}$. Напомним, что $\Sigma \subset\left|-n K_{V}+l F\right|, l \in \mathbb{Z}_{+}$.

ПрЕДЛОЖЕНИЕ 2.2. Имеет место неравенство

$$
\sum_{t \in \mathbb{P}^{1}} \max _{E \in \mathscr{M}_{t}} \frac{e(E)}{\nu_{E}\left(F_{t}\right)}>l .
$$

ДоКАЗАТЕЛЬСтво. Пусть $\widetilde{D} \in \widetilde{\Sigma}-$ общий дивизор, т. е. собственный прообраз некоторого дивизора $D \in \Sigma$ общего положения на $\widetilde{V}$. По предположению, линейная система $|\widetilde{D}+n \widetilde{K}|$ (где $\widetilde{K}-$ канонический класс многообразия $\widetilde{V}$ ) пуста. Поэтому пуста линейная система

$$
\left|l F-\sum_{E \in \mathscr{M}} e(E) E\right| .
$$

С другой стороны, для $E \in \mathscr{M}_{t}$ по построению дивизор $F_{t}-\nu_{E}\left(F_{t}\right) E$ эффективен, так что эффективен и дивизор

$$
\sum_{t \in \mathbb{P}_{1}}\left[\left(\max _{E \in \mathscr{M}_{t}} \frac{e(E)}{\nu_{E}\left(F_{T}\right)}\right) F_{t}-\sum_{E \in \mathscr{M}_{t}} e(E) E\right] .
$$

Отсюда немедленно получаем неравенство (15). Предложение 2.2 доказано.

1.2. Структура последовательности раздутий. Рассмотрим самопересечение линейной системы $\Sigma$, т. е. эффективный алгебраический цикл $Z=$ $\left(D_{1} \circ D_{2}\right)$, где $D_{1}, D_{2} \in \Sigma$ - общие дивизоры. Пусть $Z=Z^{v}+Z^{h}$ - разложение цикла $Z$ на вертикальную $\left(Z^{v}\right)$ и горизонтальную $\left(Z^{h}\right)$ части. Для цикла $Z^{v}$ имеем дальнейшее разложение

$$
Z^{v}=\sum_{t \in \mathbb{P}^{1}} Z_{t}^{v}, \quad \operatorname{Supp} Z_{t}^{v} \subset F_{t} .
$$

Пусть $E \in \mathscr{M}_{t}-$ некоторая максимальная особенность над точкой $t \in \mathbb{P}^{1}$. Для доказательства теоремы 2.1 , (iii) применим к эффективному циклу $Z_{t}^{v}+Z^{h}$ технику подсчета кратностей [1; п. 2.2]. Прежде всего, необходимо выяснить структуру последовательности раздутий, разрешающей особенность $E$. Это означает: выделить в последовательности раздутий сегменты, влияющие на кратности циклов $Z_{t}^{v}, Z^{h}$ и $\left(Z^{h} \circ F_{t}\right)$ (пересечение горизонтального цикла со слоем всегда корректно определено). 
Пока речь идет о фиксированной особенности $E$, символы $t$ и $E$ для упрощения обозначений опускаем, например, пишем $F$ вместо $F_{t}, Z^{v}$ вместо $Z_{t}^{v}$, $e$ вместо $e(E)$ и т. д.

Итак, рассмотрим последовательность раздутий (14), связанную с $E$. Как обычно, добавление верхнего индекса $j$ означает операцию взятия собственного прообраза на $V_{j}$, например, $F^{j} \subset V_{j}-$ собственный прообраз слоя $F$ и т. д. Положим

$$
N_{f}=\max \left\{i \mid B_{i-1} \subset F^{i-1}\right\}
$$

Поскольку $\varphi_{i, i-1}\left(B_{i}\right)=B_{i-1}$ для любого $i=1, \ldots, N-1$, коразмерности $\operatorname{codim} B_{i}$ не возрастают. Положим

$$
L=\max \left\{i \mid \operatorname{codim} B_{i-1} \geqslant 3\right\} \leqslant N
$$

Введем следующие обозначения: при $i \in\{1, \ldots, L\}$

$$
m_{i}^{h}=\operatorname{mult}_{B_{i-1}}\left(Z^{h}\right)^{i-1}, \quad m_{i}^{v}=\operatorname{mult}_{B_{i-1}}\left(Z^{v}\right)^{i-1},
$$

где $m_{i}^{h(v)} \leqslant m_{i-1}^{h(v)}$ для $i=2, \ldots, L$. Отметим, что в силу предположения слой $F$ можно считать неособым в общей точке $B$ и поэтому собственный прообраз $F^{i}$ неособ в общей точке каждого подмногообразия $B_{i}$, если $B_{i} \subset F^{i}$. Таким образом, для $i \in\left\{1, \ldots, N_{f}\right\}$ имеем $\operatorname{mult}_{B_{i-1}} F^{i-1}=1$. При $i>N_{f}$, очевидно, mult $_{B_{i-1}} F^{i-1}=0$. Тем более, $m_{i}^{v}=0$ при $i>N_{f}$ (если $N_{f}<L$ ). Теперь техника подсчета кратностей $[1 ;$ п. 2.2$]$ в сочетании с условием

$$
\sum_{i=1}^{K} p_{i} \nu_{i}=n \sum_{i=1}^{K} p_{i} \delta_{i}+e
$$

$e>0$, дает неравенство

$$
\sum_{i=1}^{L} p_{i} m_{i}^{h}+\sum_{i=1}^{\min \left\{N_{f}, L\right\}} p_{i} m_{i}^{v} \geqslant \sum_{i=1}^{N} p_{i} \nu_{i}^{2} \geqslant \frac{\left(n \sum_{i=1}^{N} p_{i} \delta_{i}+e\right)^{2}}{\sum_{i=1}^{N} p_{i}}
$$

где, напомним, $p_{i}$ - это число путей в графе $\Gamma$ разрешения максимальной особенности $E$ из вершины $E_{N}$ в $E_{i}$.

$\mathrm{K}$ сожалению, оценка (16) недостаточно сильная для наших целей (ее хватило бы в предположении выполнения стандартного $K^{2}$-условия, $K_{V}^{2} \notin \operatorname{Int} A_{+}^{2} V$, но мы предполагаем выполненным более слабое $K^{2}$-условие глубины 2). Необходим более тонкий анализ разрешения особенности $E$. Положим

$$
\Sigma_{l}=\sum_{i=1}^{L} p_{i}, \quad \Sigma_{u}=\sum_{i=L+1}^{N} p_{i}, \quad \Sigma_{f}=\sum_{i=1}^{\min \left\{N_{f}, L\right\}} p_{i}
$$

Отметим, что $\nu_{E}(F)=\sum_{i=1}^{N_{f}} p_{i} \geqslant \Sigma_{f}$. Очевидно, $m_{i}^{h} \leqslant m_{h}=m_{1}^{h}=\operatorname{mult}_{B} Z^{h}$. Положим также $d_{h}=\operatorname{deg} Z^{h}, d_{v}=\operatorname{deg} Z_{t}^{v}$. 
Разобьем теперь множество раздутий на несколько подмножеств. Прежде всего, выделим раздутия подмногообразий $B_{i-1}$ коразмерности три. Положим

$$
\begin{aligned}
J_{s} & =\left\{i \mid 1 \leqslant i \leqslant K, \operatorname{codim} B_{i-1} \geqslant 4\right\}, \\
J_{m} & =\left\{i \mid 1 \leqslant i \leqslant K, \operatorname{codim} B_{i-1}=3\right\}, \\
J_{u} & =\{i \mid L+1 \leqslant i \leqslant K\}, \quad J_{l}=J_{s} \cup J_{m} .
\end{aligned}
$$

В свою очередь, разобьем множество $J_{m}$ на два непересекающихся подмножества: $J_{m}=J_{m}^{+} \sqcup J_{m}^{-}$, где

$$
J_{m}^{+}=\left\{i \in J_{m} \mid B_{i-1} \subset F^{i-1}\right\},
$$

a $J_{m}^{-}=J_{m} \backslash J_{m}^{+}=\left\{i \in J_{m} \mid B_{i-1} \not \subset F^{i-1}\right\}$. Может оказаться, что $J_{m}^{+}$или $J_{m}^{-}$ (или все множество $J_{m}$ ) пусто. Положим, далее,

$$
\Sigma_{s}=\sum_{i \in J_{s}} p_{i}, \quad \Sigma_{m}^{ \pm}=\sum_{i \in J_{m}^{ \pm}} p_{i}, \quad \Sigma_{m}=\Sigma_{m}^{+}+\Sigma_{m}^{-},
$$

символ $\Sigma_{u}$ сохраняет прежний смысл. Очевидно, что $\Sigma_{l}=\Sigma_{s}+\Sigma_{m}$.

Теперь неравенство (16) можно переписать в виде

$$
\sum_{i \in J_{l}} p_{i} m_{i}^{h}+\sum_{i \in J_{s} \cup J_{m}^{+}} p_{i} m_{i}^{v} \geqslant \frac{\left(\left(3 \Sigma_{s}+2 \Sigma_{m}+\Sigma_{u}\right) n+e\right)^{2}}{\Sigma_{s}+\Sigma_{m}+\Sigma_{u}} .
$$

Следующий шаг - оценка горизонтальных кратностей $m_{i}^{h}$.

ПРЕДЛОЖЕНИЕ 2.3. Имеет место неравенство

$$
\sum_{i \in J_{s} \cup J_{m}^{+}} p_{i} m_{i}^{h} \leqslant \Sigma_{s} \operatorname{mult}_{B}\left(Z^{h} \circ F\right) .
$$

1.3. Кратности горизонтальных циклов. Докажем предложение 2.3. Приведенные ниже рассуждения справедливы с очевидными упрощениями в случае, когда $J_{m}^{+}=\varnothing$. Поэтому предполагаем, что $J_{m}^{+} \neq \varnothing$, так что, в частности, $J_{s} \subset\left\{1, \ldots, N_{f}\right\}$.

Рассмотрим сначала следующую общую ситуацию. Пусть $Y \subset V$ - неприводимое горизонтальное подмногообразие коразмерности $2, Y^{i} \subset V_{i}$ - его собственный прообраз,

$$
m_{Y}(i)=\operatorname{mult}_{B_{i-1}} Y^{i-1}
$$

- соответствующая кратность. Положим $Y_{F}=(Y \circ F)$ - эффективный цикл коразмерности два в слое $F, Y_{F}^{i} \subset V_{i}$ - его собственный прообраз,

$$
m_{Y, F}(i)=\operatorname{mult}_{B_{i-1}} Y_{F}^{i-1} .
$$

Символ о обозначает операцию взятия алгебраического цикла теоретико-схемного пересечения. Поскольку носитель цикла $Y_{F}$ содержится в слое $F$, то числа $m_{Y, F}(i)$ обращаются в нуль при $i \in J_{m}^{-}$. 
ЛЕмма 2.2. Имеет место оценка

$$
\sum_{i \in J_{s} \cup J_{m}^{+}} p_{i} m_{Y}(i) \leqslant \sum_{i \in J_{s}} p_{i} m_{Y, F}(i) .
$$

Прежде чем начинать доказательство, напомним некоторые факты, непосредственно вытекающие из элементарной теории пересечений [44]. Отметим, что здесь речь идет о пересечении дивизора и подмногообразия, а не двух дивизоров, как в [1; гл. 2]. Пусть $X$ - произвольное гладкое многообразие, $B \subset X, B \not \subset \operatorname{Sing} X$ - неприводимое подмногообразие коразмерности $\geqslant 2$, $\sigma_{B}: X(B) \rightarrow X$ - его раздутие, $E(B)=\sigma_{B}^{-1}(B)-$ исключительный дивизор. Пусть

$$
Z=\sum m_{i} Z_{i}, \quad Z_{i} \subset E(B)
$$

- цикл размерности $k, k \geqslant \operatorname{dim} B$. Определим степенъ цикла $Z$, полагая

$$
\operatorname{deg} Z=\sum_{i} m_{i} \operatorname{deg}\left(Z_{i} \cap \sigma_{B}^{-1}(b)\right),
$$

где $b \in B$ - точка общего положения, $\sigma_{B}^{-1}(b) \cong \mathbb{P}^{\operatorname{codim} B-1}$ и степень справа есть обычная степень в проективном пространстве.

Отметим, что $\operatorname{deg} Z_{i}=0$ тогда и только тогда, когда $\sigma_{B}\left(Z_{i}\right)-$ собственное замкнутое подмножество подмногообразия $B$.

Теперь пусть $D$ - простой дивизор Вейля на $X$ и $Y \subset X$ - некоторое неприводимое подмногообразие размерности $l \leqslant \operatorname{dim} X-1$. Предположим, что $Y \not \subset D$ и что $\operatorname{dim} B \leqslant l-1$. Собственные прообразы дивизора $D$ и подмногообразия $Y$ на $X(B)$ обозначим символами $D^{B}$ и $Y^{B}$ соответственно.

Лемма 2.3. (i) Предположим, что $\operatorname{dim} B \leqslant l-2$. Тогда

$$
D^{B} \circ Y^{B}=(D \circ Y)^{B}+Z
$$

где $\operatorname{Supp} Z \subset E(B) u$

$$
\operatorname{mult}_{B}(D \circ Y)=\operatorname{mult}_{B} D \cdot \operatorname{mult}_{B} Y+\operatorname{deg} Z .
$$

(ii) Предположим, что $\operatorname{dim} B=l-1$. Тогда

$$
D^{B} \circ Y^{B}=Z+Z_{1}
$$

где $\operatorname{Supp} Z \subset E(B), \operatorname{Supp} \sigma_{B}\left(Z_{1}\right)$ не содержит $B u$

$$
D \circ Y=\left[\operatorname{mult}_{B} D \cdot \operatorname{mult}_{B} Y+\operatorname{deg} Z\right] B+\left(\sigma_{B}\right)_{*} Z_{1} .
$$

ДокАЗАТЕЛЬСтво легко получается применением стандартной теории пересечений [44]. 
1.4. Техника подсчета кратностей. Построим последовательность эффективных циклов коразмерности три на многообразиях $V_{i}$, полагая

$$
\begin{aligned}
& Y \circ F=Z_{0} \quad\left(=Y_{F}\right), \\
& Y^{1} \circ F^{1}=Z_{0}^{1}+Z_{1}, \\
& \ldots \ldots \ldots \ldots \ldots \cdots \cdots \cdots \cdots \cdots \cdots \cdots \\
& Y^{i} \circ F^{i}=\left(Y^{i-1} \circ F^{i-1}\right)^{i}+Z_{i},
\end{aligned}
$$

$i \in J_{s}$, где $\operatorname{Supp} Z_{i} \subset E_{i}$. Таким образом, для любого $i \in J_{s}$ получаем:

$$
Y^{i} \circ F^{i}=Y_{F}^{i}+Z_{1}^{i}+\cdots+Z_{i-1}^{i}+Z_{i} .
$$

Для любых $j>i, j \in J_{s}$ положим $m_{i, j}=\operatorname{mult}_{B_{j-1}} Z_{i}^{j-1}$ (кратность неприводимого подмногообразия вдоль меньшего подмногообразия понимается в обычном смысле; для произвольного цикла продолжаем кратность по линейности).

Положим теперь $d_{i}=\operatorname{deg} Z_{i}$. Получаем следующую систему равенств:

$$
\begin{aligned}
& m_{Y}(1)+d_{1}=m_{Y, F}(1), \\
& m_{Y}(2)+d_{2}=m_{Y, F}(2)+m_{1,2}, \\
& \ldots \ldots \ldots \ldots \ldots \ldots \ldots \ldots \ldots \ldots \ldots \ldots \ldots \ldots \ldots \\
& m_{Y}(i)+d_{i}=m_{Y, F}(i)+m_{1, i}+\cdots+m_{i-1, i},
\end{aligned}
$$

$i \in J_{s}$. Полагая $S=\max \left\{i \in J_{s}\right\}$, рассмотрим последнее равенство в этой последовательности:

$$
m_{Y}(S)+d_{S}=m_{Y, F}(S)+m_{1, S}+\cdots+m_{S-1, S} .
$$

Если $J_{m}^{+} \neq \varnothing$, то, согласно части (ii) леммы 2.3, имеем

$$
d_{S} \geqslant \sum_{i \in J_{m}^{+}} m_{Y}(i) \operatorname{deg}\left(\varphi_{i-1, S}\right)_{*} B_{i-1} \geqslant \sum_{i \in J_{m}^{+}} m_{Y}(i) .
$$

Слегка модифицируя определение 2.6 в [1], назовем функцию $a: J_{s} \rightarrow \mathbb{R}_{+}$называется согласованной со структурой графа, если

$$
a(i) \geqslant \sum_{j \rightarrow i, j \in J_{s}} a(j)
$$

для любого $i \in J_{s}$. (По сравнению с упомянутым определением, меняется область определения функции.)

Фактически мы будем использовать только одну функцию, согласованную со структурой графа, а именно $a(i)=p_{i}$.

ПРЕДЛОЖЕНИЕ 2.4. Пустъ $а(\cdot)$ - функиия, согласованная со структурой графа. Тогда имеет место неравенство

$$
\sum_{i \in J_{s}} a(i) m_{Y, F}(i) \geqslant \sum_{i \in J_{s}} a(i) m_{Y}(i)+a(S) \sum_{i \in J_{m}^{+}} m_{Y}(i) .
$$


Доказательство проводится дословно тем же способом, что и в случае двух дивизоров $([1 ; \S 2.2])$ : умножим $i$-е равенство на $a(i)$ и сложим их все вместе. Справа для любого $i \geqslant 1$ получим выражение

$$
\sum_{j \geqslant i+1} a(j) m_{i, j}
$$

В левой части для любого $i \geqslant 1$ получаем слагаемое $a(i) d_{i}$. Далее, согласно лемме 2.3 в [1], если $m_{i, j}>0$, то $j \rightarrow i$.

Следующий стандартный шаг - сравнение кратностей $m_{i, j}$ со степенями.

Лемма 2.4. Для любых $i<j \in J_{s}$ имеем $m_{i, j} \leqslant d_{i}$.

ДокАзАтЕЛЬство. Если $m_{i, j}=0$, то доказывать нечего. В противном случае $j \rightarrow i$ и необходимо доказать, что

$$
\operatorname{mult}_{B_{j-1}} Z_{i}^{j-1} \leqslant \operatorname{deg} Z_{i}
$$

Учитывая, что отображения $\varphi_{a, b}: B_{a} \rightarrow B_{b}$ сюръективны, достаточно доказать неравенство

$$
\operatorname{mult}_{\left[B_{j-1} \cap \varphi_{i, i-1}^{-1}(t)^{j-1}\right]}\left[Z_{i} \cap \varphi_{i, i-1}^{-1}(t)\right]^{j-1} \leqslant \operatorname{deg}\left[Z_{i} \cap \varphi_{i, i-1}^{-1}(t)\right],
$$

где $t \in B_{i-1}$ - точка общего положения. Учитывая, что $\varphi_{i, i-1}^{-1}(t)$ есть проективное пространство $\mathbb{P}^{\operatorname{codim} B_{i-1}-1}$, получаем, что справа в (23) стоит обычная степень гиперповерхности в проективном пространстве, а множество $\left[Z_{i} \cap\right.$ $\left.\varphi_{i, i-1}^{-1}(t)\right]^{j-1}$ получается из этой гиперповерхности конечной последовательностью раздутий $\varphi_{s, s-1}, s=i+1, \ldots, j-1$, ограниченных на $\varphi_{i, i-1}^{-1}(t)$. Учитывая, что кратности при раздутиях не возрастают, сводим утверждение к очевидному случаю гиперповерхности в проективном пространстве. Лемма доказана.

В результате получаем следующую оценку:

$$
\sum_{j \geqslant i+1} a(j) m_{i, j}=\sum_{j \geqslant i+1, m_{i, j} \neq 0} a(j) m_{i, j} \leqslant d_{i} \sum_{j \rightarrow i} a(j) \leqslant a(i) d_{i} .
$$

В силу сказанного выше, можно выбросить справа все слагаемые $m_{i, *}, i \geqslant 1$, а слева - все слагаемые $d_{i}, i \geqslant 1$, заменяя при этом знак равенства $=$ на знак неравенства $\leqslant$. Что и требовалось доказать.

Полагая в неравенстве $(22) a(i)=p_{i}$ и замечая, что для $j \geqslant S$ имеем $p_{j} \leqslant p_{S}$, завершаем доказательство леммы 2.2.

Закончим доказательство предложения 2.3.

Очевидно, что неравенство (21) остается справедливым, если $Y$ - эффективный горизонтальный цикл коразмерности 2 на $V$, т. е. каждая неприводимая компонента цикла $Y$ - горизонтальное подмногообразие. При этом формулы (19), (20) продолжаются по линейности на множество всех эффективных горизонтальных циклов, а левая и правая части неравенства $(21)$ линейны по $m_{Y}(\cdot)$, $m_{Y, F}(\cdot)$ соответственно.

Положим теперь $Y=Z^{h}$ и учтем, что $m_{Y, F}(i) \leqslant \operatorname{mult}_{B}\left(Z^{h} \circ F\right)$ для $i \geqslant 1$. Этим предложение 2.3 доказано. 
1.5. Сверхмаксимальная особенность. Полученные выше оценки применим к некоторой максимальной особенности $E \in \mathscr{M}$, удовлетворяющей, кроме неравенства Нётера-Фано, некоторому дополнительному условию, сформулированному ниже в предложении 2.5. Такие особенности называются сверxмаксимальными. Поскольку по предположению выполнено $K^{2}$-условие глубины 2 , для горизонтальной части самопересечения линейной системы $\Sigma$ имеем

$$
Z^{h} \sim n^{2} K_{V}^{2}+\alpha H_{F}
$$

где коэффициент $\alpha \in \mathbb{Z}$ удовлетворяет неравенству $\alpha \geqslant-2 n^{2}$. Следовательно, для вертикальной компоненты имеем

$$
Z^{v} \sim(2 n l-\alpha) H_{F},
$$

откуда

$$
\operatorname{deg} Z^{v}=\sum_{t \in \mathbb{P}^{1}} \operatorname{deg} Z_{t}^{v} \leqslant\left(2 n l+2 n^{2}\right) \operatorname{deg} V .
$$

ПРЕДЛОЖЕНИЕ 2.5. Для некоторой точки $t \in \mathbb{P}^{1}$ существует максимальная особенность $E \in \mathscr{M}_{t} \neq \varnothing$, удовлетворяющая оценке

$$
e(E)>\frac{\nu_{E}\left(F_{t}\right)}{2}\left(\frac{\operatorname{deg} Z_{t}^{v}}{n \operatorname{deg} V}-2 n\right) .
$$

ДоказАтельство. Рассмотрим неравенства (15) и (24). Заменяя число $l$ в правой части неравенства (24) левой частью неравенства (15), получим

$$
\sum_{t \in \mathbb{P}^{1}}\left[\operatorname{deg} Z_{t}^{v}-2 n \operatorname{deg} V \max _{\left\{E \in \mathscr{M}_{t}\right\}} \frac{e(E)}{\nu_{E}\left(F_{t}\right)}\right]<2 n^{2} \operatorname{deg} V,
$$

откуда немедленно следует наше предложение.

ЗАмЕчАниЕ 2.1. Если имеется несколько максимальных особенностей, центры которых лежат в слоях над различными точками $t_{1}, \ldots, t_{k}$, то предложение 2.5 можно усилить: существует максимальная особенность $E \in \mathscr{M}_{t}$, $t \in\left\{t_{1}, \ldots, t_{k}\right\}$, удовлетворяющая оценке

$$
e(E)>\frac{\nu_{E}\left(F_{t}\right)}{2}\left(\frac{\operatorname{deg} Z_{t}^{v}}{n \operatorname{deg} V}-\frac{2 n}{k}\right) .
$$

Таким образом, мы рассматриваем наихудшую возможность, полагая $k=1$.

Пусть $o \in B$ - точка общего положения. Поскольку по предположению $o \in F$ - гладкая точка слоя, выполнены условия $(f)$ и $(v)$. Из неравенства (13) немедленно получаем оценку

$$
\sum_{i \in J_{s} \cup J_{m}^{+}} p_{i} m_{i}^{h} \leqslant 4 n^{2} \Sigma_{s}
$$

Поскольку $m_{i}^{h} \leqslant m_{1}^{h} \leqslant 4 n^{2}$, получаем неравенство

$$
\sum_{i \in J_{l}} p_{i} m_{i}^{h} \leqslant 4 n^{2}\left(\Sigma_{s}+\Sigma_{m}^{-}\right) .
$$

Это и есть необходимая оценка на особенности горизонтальной компоненты $Z^{h}$. 
Рассмотрим теперь вертикальную компоненту $Z^{v}$. В силу условия $(v)$ имеет место неравенство

$$
m_{i}^{v} \leqslant m_{1}^{v} \leqslant \frac{2}{\operatorname{deg} V} d_{v}
$$

С другой стороны, из $K^{2}$-условия глубины 2 получаем оценку

$$
\frac{d_{v}}{\operatorname{deg} V}<\frac{2 e n}{\nu_{E}(F)}+2 n^{2}
$$

Комбинируя (27) и (28), получаем неравенство

$$
\sum_{i \in J_{s} \cup J_{m}^{+}} p_{i} m_{i}^{v}<2 n\left(\frac{2 e}{\nu_{E}(F)}+2 n\right)\left(\Sigma_{s}+\Sigma_{m}^{+}\right) .
$$
Учитывая, что по определению $\nu_{E}(F)=\sum_{i=1}^{k} p_{i} \mu_{i} \geqslant \Sigma_{s}+\Sigma_{m}^{+}$, получаем оконча-
тельно:

$$
\sum_{i \in J_{s} \cup J_{m}^{+}} p_{i} m_{i}^{v}<4 n e+4 n^{2}\left(\Sigma_{s}+\Sigma_{m}^{+}\right) .
$$

Теперь из неравенств (16), (26) и (29) вытекает следующая оценка:

$$
\begin{gathered}
\left(4 n^{2}\left(\Sigma_{s}+\Sigma_{m}^{-}\right)+4 n e+4 n^{2}\left(\Sigma_{s}+\Sigma_{m}^{+}\right)\right)\left(\Sigma_{s}+\Sigma_{m}+\Sigma_{u}\right) \\
>\left(\left(3 \Sigma_{s}+2 \Sigma_{m}+\Sigma_{u}\right) n+e\right)^{2} .
\end{gathered}
$$

Учитывая, что $\Sigma_{m}=\Sigma_{m}^{+}+\Sigma_{m}^{-}$, после несложных арифметических преобразований получаем неравенство

$$
\left(n\left(\Sigma_{s}-\Sigma_{u}\right)+e\right)^{2}<0 .
$$

Противоречие.

Доказательство утверждения (iii) теоремы 2.1 завершено.

\section{§ 2. Многообразия с пучком полных пересечений Фано}

Цель данного параграфа - объяснить ключевые шаги доказательства теорем 1.5 и 1.6. Технической основой доказательства является теорема 2.1, так что работа сводится к проверке условий $(f),(v)$ и исключению бесконечно близких максимальных особенностей, лежащих над особой точкой слоя. Проверка $K^{2}$-условия глубины 2 и $K$-условия несложна (см. предложения $1.4,1.5$ ) и мы ее опустим.

2.1. Расслоения на полные пересечения Фано. Докажем теорему 1.5. Поскольку расслоение $V / \mathbb{P}^{1}$ достаточно общее, можно предполагать, что каждый слой $F$ в каждой точке удовлетворяет условию регулярности, сформулированному в определении 3.4 обзора [1], т. е. набор многочленов

$$
\left\{q_{i, j} \mid 1 \leqslant i \leqslant k, 1 \leqslant j \leqslant d_{i},(i, j) \neq\left(k, d_{k}\right)\right\}
$$

образует регулярную последовательность. Тогда [32] или [1; гл. $3, \S 2]$ дают условие $(f)$. Докажем условие $(v)$. Пусть $Y \subset F=F_{t}$ - простой дивизор, 
$o \in Y$ - некоторая точка. Возьмем общую гиперплоскость $H \subset \mathbb{P}$, касательную к $F$ в точке $o$, т.е. $H \supset T_{o} F$. Пусть $T=H \cap F$. В силу общности имеем $Y \neq T$, так что $Y_{T}=(Y \circ T)$ есть корректно определенный эффективный цикл коразмерности два на $F$, и, более того,

$$
\frac{\text { mult }_{o}}{\operatorname{deg}} Y_{T} \geqslant 2 \frac{\text { mult }_{o}}{\operatorname{deg}} Y .
$$

Теперь из условия $(f)$ следует $(v)$.

Для доказательства теоремы 1.5 остается проверить, что центр максимальной особенности системы $\Sigma \subset\left|-n K_{V}+l F\right|$ (или пары $\left.\left(V, n^{-1} \Sigma\right)\right)$ не может быть особой точкой слоя. Таких точек на многообразии $V$ конечное число, так что можно предполагать выполненными дополнительные условия общности положения. Сформулируем эти условия.

Пусть, как в $[1 ;$ гл. $3, \S 2],\left(z_{1}, \ldots, z_{M+k}\right)$ есть система аффинных координат на $\mathbb{P}=\mathbb{P}^{M+k}$ с началом в точке $o \in V$, которая является особой точкой слоя $F \ni o, F \subset \mathbb{P}$. По предположению, $o \in F-$ невырожденная квадратичная особенность. Предположим дополнительно, что система однородных уравнений $\left\{q_{i, j}=0 \mid(i, j) \neq\left(k, d_{k}\right)\right\}$ задает замкнутое множество размерности два в $\mathbb{C}^{M+k}$ (соответственно кривую в $\mathbb{P}^{M+k-1}$ ) такое, что линейная оболочка каждой его неприводимой компоненты есть линейное подпространство

$$
T=\left\{q_{1,1}=q_{2,1}=\cdots=q_{k, 1}=0\right\} .
$$

Отметим, что если $x \in F$ - особенность, то линейные формы $q_{i, 1}, i=1, \ldots, k$, линейно зависимы. Регулярность точки $o \in F$ означает, что, удаляя из набора (30) в точности одну линейную форму, скажем, $q_{1, e}$, получим регулярную последовательность, т. е. система уравнений

$$
\left\{q_{i, j}=0 \mid(i, j) \notin\left\{(1, e),\left(k, d_{k}\right)\right\}\right\}
$$

задает двумерное множество в $\mathbb{C}^{M+k}$ (кривую в пространстве $\mathbb{P}^{M+k-1}$ ). В частности, $\operatorname{codim} T=k-1$ и касательный конус $T_{x} F \subset T$ есть невырожденная квадрика. Более того, из условия регулярности следует, что, заменяя в набоpe $(30)$ линейную форму $q_{1, e}$ произвольной линейной формой $l\left(z_{1}, \ldots, z_{M+k-1}\right)$ такой, что $\left.l\right|_{T} \not \equiv 0$, получим регулярную последовательность, поскольку ни одна из компонент замкнутого множества (31) не содержится в гиперплоскости $l=0$.

Предположим теперь, что особая точка слоя $o \in F$ есть центр максимальной особенности. Пусть $\lambda: F^{+} \rightarrow F-$ раздутие точки $о$ и $\lambda^{-1}(o)=E^{+} \subset F^{+}-$ исключительный дивизор. Раздутие $\lambda$ можно рассматривать как ограничение раздутия $\lambda_{\mathbb{P}}: \mathbb{P}^{+} \rightarrow \mathbb{P}$ точки $о$ на $\mathbb{P}$, так что $E^{+} \subset E-$ неособая квадрика размерности $M-1$, где $E=\lambda_{\mathbb{P}}^{-1}(o) \cong \mathbb{P}^{M+k-1}$ - исключительный дивизор.

ПредлОЖениЕ 2.6. Существует гиперплоское сечение $B$ квадрики $E^{+} \subset E$, удовлетворяющее неравенству

$$
\operatorname{mult}_{B}\left(\lambda^{*} \Sigma_{F}\right)>2 n .
$$


ДокАЗАТЕльство дано в $§ 2$ главы 3, где собраны все факты, относящиеся к принципу связности Шокурова-Коллара.

Пусть $D \in \Sigma_{F}=\left.\Sigma\right|_{F}$ - эффективный дивизор на $F, D \in\left|n H_{F}\right|$. Для собственного прообраза $D^{+} \subset F^{+}$имеем $\lambda^{*} D=D^{+}+\left(2^{-1}\right.$ mult $\left._{o} D\right) E^{+}$. Принимая во внимание, что mult $_{B} E^{+}=1$, получаем из предложения 2.6 неравенство

$$
\operatorname{mult}_{o} D+2 \operatorname{mult}_{B} D^{+}>4 n .
$$

Пусть $H \subset \mathbb{P}$ - общая гиперплоскость, содержащая точку о и высекающая $B$, т. е. $H^{+} \cap E^{+}=\left(H^{+} \cap E\right) \cap E^{+}=B$, где $H^{+} \subset \mathbb{P}^{+}$- собственный прообраз. Положим $T=H \cap F$. Многообразие $T$ есть полное пересечение типа $\left(d_{1}, \ldots, d_{k}\right)$ в $H=\mathbb{P}^{M+k-1}$ с изолированной квадратичной особенностью в точке $o$. Эффективный дивизор $D_{T}=(D \circ T)$ на $T$ удовлетворяет неравенству

$$
\text { mult }_{o} D_{T}>4 n \text {. }
$$

Очевидно, что $D_{T} \in\left|n H_{T}\right|$, где $H_{T}$ есть гиперплоское сечение многообразия $T \subset \mathbb{P}^{M+k-1}$. По линейности, можно предполагать, что $D_{T}-$ простой дивизор, т. е. неприводимое подмногообразие коразмерности единица.

Теперь получаем противоречие, повторяя дословно рассуждения [1; гл. 3 , $\S 2]:$ пересекая дивизор $D_{T}$ с гиперкасательными дивизорами, строим кривую $C \subset T$, удовлетворяющую неравенству mult ${ }_{o} C>\operatorname{deg} C$, что, конечно, невозможно. Остается проверить, что техника гиперкасательных дивизоров применима к нашему случаю. В [1; гл. $3, \S 2]$ использовались два факта:

1) справедливость условия регулярности для полного пересечения $F$ в рассматриваемой точке;

2) неприводимость пересечения $F \cap T_{o} F$ (она выводилась из неравенства $k<2^{-1} \operatorname{dim} F$ с помощью теоремы Лефшеца).

Рассуждения из $[1 ;$ гл. $3, \S 2]$ работают в нашем случае, если выполнены условия 1) и 2).

Что касается условия регулярности, оно справедливо в нашем случае благодаря усиленному условию регулярности в особой точке, сформулированному выше. Точнее, гиперплоское сечение $T=H \cap F$ удовлетворяет обычному условию регулярности для любой гиперплоскости $H \not \supset T_{o} F$.

Рассмотрим условие 2). Напомним, что в рассматриваемом нами случае $\operatorname{codim} T_{o} F=k-1$ (точка $o \in F$ - особая). Вместо условия 2) нам нужен следующий факт: пересечение

$$
T \cap T_{o} F=H \cap F \cap T_{o} F
$$

неприводимо (и в силу условия регулярности автоматически имеет кратность точно $2^{k}$ в точке $о$ ). Это верно снова по теореме Лефшеца ввиду неравенства $k<2^{-1} \operatorname{dim} F$, поскольку $o \in F-$ невырожденная двойная точка. Этим доказательство теоремы 1.5 завершается.

2.2. Расслоения на циклические накрытия Фано. Докажем теорему 1.6. Условия $(f)$ и $(v)$ для достаточно общих циклических накрытий Фано проверяются тем же способом, что и для полных пересечений выше, с учетом 
дополнительных гиперкасательных дивизоров, описанных в [1; гл. $3, \S 2]$. Детали см. в [8]. Теперь достаточно показать, что центр максимальной особенности не может быть особой точкой слоя $o \in F$.

Пусть $\sigma: F \rightarrow G \subset \mathbb{P}=\mathbb{P}^{M+1}$ - реализация слоя в виде $K$-листного циклического накрытия. Для общего многообразия $V$ особая точка $o \in F$ может быть строго одного из двух типов:

- когда гиперповерхность $G$ имеет в точке $p=\sigma(o)$ невырожденную квадратичную особенность (при этом точка $p$ не лежит на дивизоре ветвления);

- когда гиперповерхность $G$ неособа в точке $p=\sigma(o)$, но дивизор ветвления $W \cap G$, где $W=W_{K l} \subset \mathbb{P}$ - гиперповерхность степени $K l$, имеет в точке $p$ квадратичную особенность.

Особенность $o \in F$ первого типа исключается как возможный центр максимальной особенности точно таким же способом, как в п. 2.1 (опять же, с учетом дополнительных гиперкасательных дивизоров для $K$-листного накрытия). Отсылая читателя к [8] за подробностями, рассмотрим особенность $o \in F$ второго типа. В этом случае для исключения максимальной особенности нужен другой метод.

Прежде всего, сформулируем условие регулярности для особенности второго типа. Вводя новую координату $u$ веса $l$, реализуем слой $F$ как полное пересечение типа $m \cdot K l$ во взвешенном проективном пространстве

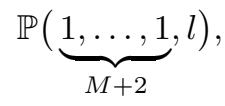

а именно, $F$ задается системой уравнений

$$
\left\{\begin{array}{l}
f\left(x_{0}, \ldots, x_{M+1}\right)=0 \\
u^{K}=g\left(x_{0}, \ldots, x_{M+1}\right)
\end{array}\right.
$$

где $f\left(x_{*}\right)$ и $g\left(x_{*}\right)$ - однородные многочлены степеней $m$ и $K l$ соответственно. Напомним, что целые числа $m, l$ и $K$ удовлетворяют соотношению

$$
m+(K-1) l=M+1 .
$$

Пусть $F \sim(f, g)$ - наше многообразие, $o \in F$ - произвольная точка, $p=$ $\sigma(o) \in G=\{f=0\}$ - ее образ на $\mathbb{P}$. Выберем некоторую систему аффинных координат $z_{1}, \ldots, z_{M+1}$ с началом в точке $p$. Без ограничения общности можно предполагать, что $z_{i}=x_{i} / x_{0}$. Положим $y=u / x_{0}^{l}$. Тогда стандартное аффинное множество $\mathbb{A}_{\left(z_{1}, \ldots, z_{M+1}, y\right)}^{M+2}$ является картой для $\mathbb{P}(1, \ldots, 1, l)$. Злоупотребляя обозначениями, используем для неоднородных многочленов, соответствующих $f$ и $g$, те же самые символы:

$$
f=q_{1}+\cdots+q_{m}, \quad g=w_{0}+w_{1}+\cdots+w_{K l},
$$

где $q_{i}, w_{j}$ - однородные компоненты степени $i, j$ соответственно от переменных $z_{*}$, так что в указанной аффинной карте $\mathbb{A}_{\left(z_{*}, y\right)}^{M+2}$ многообразие $F$ задается парой уравнений $f=0, y^{K}=g$ (заменяющей систему (33)). Если точка $o \in F$ не лежит на дивизоре ветвления морфизма $\sigma$, то всегда предполагаем, что 
$w_{0}=1$. Если точка $p \in G$ неособа, то без ограничения общности считаем, что $q_{1} \equiv z_{M+1}$. В последнем случае полагаем

$$
\bar{q}_{i}=\left.q_{i}\right|_{\left\{z_{M+1}=0\right\}}=q_{i}\left(z_{1}, \ldots, z_{M}, 0\right)
$$

и $\bar{w}_{j}=\left.w_{j}\right|_{z_{M+1}=0}=w_{j}\left(z_{1}, \ldots, z_{M}, 0\right)$ при $i, j \geqslant 2$.

Сформулируем теперь условие регулярности для особой точки $o \in F$. Здесь $w_{0}=0, q_{1}=z_{M+1}, w_{1}=\lambda z_{M+1}$, где $\lambda \in \mathbb{C}$ - некоторая константа, т. е. точка $p \in G$ неособа. Требуется, чтобы квадратичная форма $\bar{w}_{2}\left(z_{1}, \ldots, z_{M}\right)$ имела максимальный ранг $M$ и последовательность

$$
\bar{q}_{2}, \ldots, \bar{q}_{m}, \bar{w}_{2}, \ldots, \bar{w}_{K}
$$

была регулярной в $\mathscr{O}_{o, \mathbb{C}^{M}}$, причем система однородных уравнений

$$
\bar{q}_{2}=\cdots=\bar{q}_{m}=\bar{w}_{2}=\cdots=\bar{w}_{K}=0
$$

задавала бы замкнутое алгебраическое множество в $\mathbb{C}^{M}$, ни одна неприводимая компонента которого не содержалась бы в гиперплоскости.

Очевидно, можно считать, что $\lambda \in\{0,1\}$. Итак, либо $q_{1} \equiv w_{1} \equiv z_{M+1}$, либо $q_{1} \equiv z_{M+1}$ и $w_{1} \equiv 0$. Росток многообразия $o \in F$ аналитически есть росток гиперповерхности $y^{K}=\bar{w}_{2}\left(z_{1}, \ldots, z_{M}\right)+\cdots$, где $\bar{w}_{i}, \bar{q}_{i}$ суть ограничения многочленов $w_{i}, q_{i}$ на гиперплоскость $z_{M+1}=0$, в пространстве $\mathbb{C}_{\left(z_{1}, \ldots, z_{M}, y\right)}^{M+1}$. Пусть $\varphi: F^{+} \rightarrow F-$ раздутие точки $о$ и $E \subset F^{+}-$исключительный дивизор. Из сказанного следует, что $E$ естественно реализуется как квадратичная гиперповерхность $E \subset \mathbb{P}_{\left(z_{1}: \cdots: z_{M}: y\right)}^{M}$. Пусть $\varphi_{G}: G^{+} \rightarrow G-$ раздутие точки $p \in G$ и $E_{G}=\varphi_{G}^{-1}(p) \subset G^{+}-$исключительный дивизор, $E_{G} \cong \mathbb{P}_{\left(z_{1}: \cdots: z_{M}\right)}^{M-1}$. Легко видеть, что морфизм $\sigma$ продолжается до рационального отображения $\sigma^{+}: F^{+} \rightarrow G^{+}$, причем ограничение

$$
\sigma_{E}=\left.\sigma^{+}\right|_{E}: E \rightarrow E_{G}
$$

есть проекция квадратичного конуса $E \subset \mathbb{P}^{M}$, заданного в $\mathbb{P}_{\left(z_{1}: \cdots: z_{M}: y\right)}^{M}$ уравнением $\bar{w}_{2}\left(z_{1}, \ldots, z_{M}\right)=0$, из его вершины $o^{+}=(0: \cdots: 0: 1)$ на неособую квадрику $E_{+} \subset E_{G}$, заданную в $E_{G}=\mathbb{P}^{M-1}$ тем же самым уравнением $\bar{w}_{2}=0$. Таким образом, $\sigma^{+}$стягивает образующие конуса $E$ в точки.

Согласно условию регулярности, система однородных уравнений (34) высекает на $E_{+}$(и, тем самым, на $E$ ) замкнутое алгебраическое множество, ни одна компонента которого не содержится в гиперплоскости.

Предположим, что существуют эффективный дивизор $R \in\left|n H_{F}\right|$ и гиперплоское сечение $B$ квадратичного конуса $E \subset \mathbb{P}^{M}$, удовлетворяющее неравенству

$$
\nu+\mu>2 n,
$$

где $R^{+} \in\left|n H_{F}-\nu E\right|$, т. е. $\nu=2^{-1}$ mult $_{o} R$, и $\mu=\operatorname{mult}_{B} R^{+}, R^{+} \subset F^{+}-$собственный прообраз дивизора $R$. В силу линейности неравенства (35) дивизор $R$ можно считать простым.

ЛЕмма 2.5. Имеет место оценка $\nu \leqslant 3 n / 2$. 
ДокАЗАТЕЛЬСтво. Предположим противное: $\nu>3 n / 2$. Пусть

$$
\begin{aligned}
& D_{i}=\sigma^{*}\left(\left.f_{i}\right|_{G}\right), \quad f_{i}=q_{1}+\cdots+q_{i}, \quad i=1, \ldots, m-1, \\
& \Delta_{i}=\sigma^{*}\left(\left.g_{i}\right|_{G}\right), \quad g_{i}=w_{1}+\cdots+w_{i}, \quad i=2, \ldots, K-1,
\end{aligned}
$$

- гиперкасательные дивизоры на $F, \mathscr{D}=\left\{D_{1}, \ldots, D_{m-1}, \Delta_{2}, \ldots, \Delta_{K-1}\right\}, \sharp \mathscr{D}=$ $m+K-3$. В силу условия регулярности имеем

$$
\operatorname{codim}_{O}\left(\bigcap_{D \in \mathscr{D}} D\right)=\sharp \mathscr{D} .
$$

Лемма 2.6. Простые дивизоры $D_{1}$ и $R$ различны: $D_{1} \neq R$.

ДокАЗАТЕЛЬСтво. Если $\nu \leqslant 2 n$, то $\mu \geqslant 1$, т. е. $R^{+} \supset B$. В силу условия регулярности $D_{1}^{+} \not \supset B$. Значит, $D_{1}^{+} \neq R^{+}$.

Если $\nu>2 n$, то, учитывая, что $D_{1}^{+} \in\left|H_{F}-2 E\right|$, снова получаем, что $D_{1} \neq R$. Лемма доказана.

В силу леммы 2.6 корректно определен эффективный цикл $\left(R \circ D_{1}\right)$ коразмерности два. Имеет место неравенство

$$
\frac{\text { mult }_{o}}{\operatorname{deg}}\left(R \circ D_{1}\right)>\frac{6}{m K},
$$

так что найдется неприводимое подмногообразие $Y \subset F$ коразмерности два (неприводимая компонента цикла $\left(R \circ D_{1}\right)$ ), удовлетворяющая неравенству

$$
\frac{\text { mult }_{o}}{\operatorname{deg}} Y>\frac{6}{m K} \text {. }
$$

Применяя к подмногообразию $Y$ предложение 3.2 из [1], получаем противоположное неравенство

$$
\frac{\text { mult }_{o}}{\operatorname{deg}} Y \leqslant \frac{2}{3} \cdots \frac{m-1}{m} \cdot \frac{3}{4} \cdots \frac{K-1}{K}=\frac{6}{m K} .
$$

Полученное противоречие доказывает, кроме того, что максимум отношения mult $_{o} / \operatorname{deg}$ достигается на дивизоре $\sigma^{*} D_{1}$ и равен $4 /(m K)$. Лемма доказана.

СЛЕДСТВИЕ 2.1. Имеет место неравенство $\mu>n / 2$.

Таким образом, гиперплоское сечение $B$ реально присутствует в дивизоре $R^{+} \subset F^{+}$. Теперь удобнее воспользоваться техникой гиперкасательных линейных систем (см. [1; п. 3.1]). Положим

$$
\Lambda_{i}^{G}=\left|\left(\sum_{j=1}^{i} f_{j} s_{i-j}+\sum_{j=2}^{i} g_{j} r_{i-j}\right)\right|_{G}=0 \mid,
$$

где $s_{k}, r_{k}$ пробегают множество всех однородных многочленов от $z_{1}, \ldots, z_{M+1}$ степени $k$. Далее, пусть

$$
\Lambda_{i}=\sigma^{*} \Lambda_{i}^{G} \quad \text { и } \quad \Lambda_{i}^{+}
$$


- подъем системы $\Lambda_{i}^{G}$ на $F$ и его собственный прообраз на $F^{+}$соответственно. Имеем $\Lambda_{i}^{+} \subset\left|i H_{F}-(i+1) E\right|$. Наконец, пусть

$$
\Lambda_{i}^{E}=\left.\Lambda_{i}^{+}\right|_{E}
$$

- проективизированная касательная система линейной системы $\Lambda_{i}$ в точке $o$.

Если $H_{E}-$ класс гиперплоского сечения конуса $E$, то $\Lambda_{i}^{E} \subset\left|(i+1) H_{E}\right|$. В координатном виде

$$
\Lambda_{i}^{E}=\sigma_{E}^{*}\left|\sum_{j=1}^{i} \bar{q}_{j+1} \bar{s}_{i-j}+\sum_{j=2}^{i} \bar{w}_{j+1} \bar{r}_{i-j}=0\right|,
$$

где $\bar{s}_{k}=\left.s_{k}\right|_{\left\{z_{M+1}=0\right\}}, \bar{r}_{k}=\left.r_{k}\right|_{\left\{z_{M+1}=0\right\}}$. Из этих явных представлений и условия регулярности сразу получаем, что

$$
\operatorname{codim}_{E} \operatorname{Bs} \Lambda_{i}^{E}=\min \{i, m-1\}+\min \{i, K-1\}-1,
$$

причем ни одна компонента замкнутого множества Bs $\Lambda_{i}^{E}$ не содержится в гиперплоскости. Отметим, что при $i \geqslant \max \{m, K\}-1$ имеем $\operatorname{Bs} \Lambda_{i}=\operatorname{Bs} \Lambda_{i+1}$ : это в точности замкнутое множество

$$
\bar{q}_{2}=\cdots=\bar{q}_{m}=\bar{w}_{3}=\cdots=\bar{w}_{K}=0 .
$$

Пусть

$$
\mathscr{L}=\left(D_{1}, \ldots, D_{m-1}, L_{2}, \ldots, L_{K-1}\right) \in \prod_{i=1}^{m-1} \Lambda_{i} \times \prod_{i=2}^{K-1} \Lambda_{i}
$$

- общий набор гиперкасательных дивизоров,

$$
\mathscr{L}^{+}=\left(D_{1}^{+}, \ldots, L_{K-1}^{+}\right) \quad \text { и } \quad \mathscr{L}^{E}=\left(D_{1}^{E}, \ldots, L_{K-1}^{E}\right)=\left.\mathscr{L}^{+}\right|_{E}
$$

- его собственный прообраз на $F^{+}$и ограничение на квадратичный конус $E$ соответственно.

Пусть $R_{E}=\left.R^{+}\right|_{E}$ - проективизированный касательный конус дивизора $R$ в точке $о$. В силу условия регулярности замкнутое алгебраическое множество

$$
R_{E} \cap\left(\bigcap_{i=2}^{m-1} D_{i}^{E}\right) \cap\left(\bigcap_{i=2}^{K-1} L_{i}^{E}\right)
$$

имеет коразмерность точно $m+K-2$ относительно $E$ (обратим внимание, что в (36) пропущен дивизор $\left.D_{1}^{E}\right)$.

Следовательно, корректно определен эффективный цикл

$$
Y_{E}=\left(R_{E} \circ D_{2}^{E} \circ \cdots \circ D_{m-1}^{E} \circ L_{2}^{E} \circ \cdots \circ L_{K-1}^{E}\right)
$$

коразмерности $m+K-2$ на $E$. Его $H_{E}$-степень есть

$$
\operatorname{deg} Y_{E}=\frac{1}{4} m ! K ! \operatorname{deg} R_{E}=\frac{1}{2} m ! K ! \nu .
$$


Далее, в силу условия регулярности замкнутое множество

$$
R \cap\left(\bigcap_{i=2}^{m-1} D_{i}\right) \cap\left(\bigcap_{i=2}^{K-1} L_{i}\right)
$$

имеет коразмерность $m+K-2$ относительно $F$ в окрестности точки о. Следовательно, однозначно определен эффективный алгебраический цикл $Y$ коразмерности $m+K-2$ на $F$, каждая неприводимая компонента которого содержит точку о и который совпадает с эффективным циклом

$$
\left(R \circ D_{2} \circ \cdots \circ D_{m-1} \circ L_{2} \circ \cdots \circ L_{K-1}\right)_{U}
$$

на подходящем открытом по Зарискому множестве $U \subset F$, содержащем точку о. Для общего набора $\mathscr{L}$ имеем: $Y_{E}=\left(Y^{+} \circ E\right)-$ проективизированный касательный конус к $Y$ в точке $о$. При этом, вообще говоря, для $H_{F}$-степени цикла $Y$ имеем лишь неравенство

$$
\operatorname{deg} Y \leqslant(m-1) !(K-1) ! \operatorname{deg} R=n m ! K !,
$$

поскольку на промежуточных шагах построения цикла $Y$ (дивизор $R$ последовательно пересекается с $D_{2}, L_{2}, D_{3}, L_{3}$ и т. д.) отбрасываются компоненты, не содержащие точку $о$. Отметим, что, вообще говоря, некоторые неприводимые компоненты циклов $Y$ и $Y_{E}$ могут содержаться в $D_{1}$ и $D_{1}^{E}$ соответственно. Выделим эти компоненты:

$$
Y=Z+Y^{\sharp}, \quad Y_{E}=Z_{E}+Y_{E}^{\sharp},
$$

где $\operatorname{Supp} Z \subset D_{1}$ и $Z$ - максимальный подцикл эффективного цикла $Y$ с этим свойством (т. е. ни одна неприводимая компонента цикла $Y^{\sharp}$ не содержится в $\left.D_{1}\right), Z_{E}=\left(Z^{+} \circ E\right), Y_{E}^{\sharp}=\left(\left(Y^{\sharp}\right)^{+} \circ E\right)=Y_{E}-Z_{E}$. Очевидно, что Supp $Z_{E} \subset D_{1}^{E}$, но неприводимые компоненты цикла $Y_{E}^{\sharp}$, вообще говоря, могут содержаться в $D_{1}^{E}$. Ключевое значение имеет следующий факт.

ЛЕмма 2.7. Алгебрачческий цикл

$$
Y_{E}^{\sharp}-\mu\left(B \circ D_{2}^{E} \circ \cdots \circ D_{m-1}^{E} \circ L_{2}^{E} \circ \cdots \circ L_{K-1}^{E}\right)
$$

эфбективен. В частности, имеет место неравенство

$$
\operatorname{deg} Y_{E}^{\sharp} \geqslant \frac{1}{2} m ! K ! \mu .
$$

ДокАЗАТЕЛЬство. Первое утверждение следует из того, что по построению алгебраический цикл $R_{E}-\mu B$ эффективен, так что эффективен цикл $Y_{E}-\mu B \mathscr{L}$, где $B_{\mathscr{L}}=\left(B \circ D_{2}^{E} \circ \cdots \circ L_{K-1}^{E}\right)$. Носитель цикла $B_{\mathscr{L}}$ есть замкнутое множество $\operatorname{Supp} B_{\mathscr{L}}$ чистой коразмерности $m+K-3$ относительно $E$. Для любой неприводимой компоненты $\Delta \subset \operatorname{Supp} B_{\mathscr{L}}$ имеем $\Delta \not \subset D_{1}^{E}$.

В самом деле, предположим, что это не так: $\Delta \subset D_{1}^{E}$. Тогда $\Delta \subset B$ и

$$
\Delta \subset\left(\bigcap_{i=1}^{m-1} D_{i}^{E}\right) \cap\left(\bigcap_{i=2}^{K-1} L_{i}^{E}\right),
$$

что противоречит условию регулярности. 
Следовательно, неприводимая компонента $\Delta$ цикла $B \mathscr{L}$ не может быть компонентой цикла $Z_{E}$ и, таким образом, входит только в цикл $Y_{E}^{\sharp}$. Это доказывает первое утверждение леммы. Второе утверждение тривиальным образом следует из первого. Лемма доказана.

Поскольку неприводимые компоненты цикла $Y^{\sharp}$ не содержатся в дивизоpe $D_{1}$, то корректно определен эффективный цикл $\left(Y^{\sharp} \circ D_{1}\right)$, причем выполнено неравенство

$$
\frac{\text { mult }_{o}}{\operatorname{deg}}\left(Y^{\sharp} \circ D_{1}\right) \geqslant 2 \frac{\text { mult }_{o}}{\operatorname{deg}} Y^{\sharp} .
$$

Поскольку для любого неприводимого подмногообразия $\Delta \subset F$ имеем оценку $\left(\right.$ mult $\left._{o} / \operatorname{deg}\right) \Delta \leqslant 1$, получаем

$$
\operatorname{deg} Y^{\sharp} \geqslant 2 \text { mult }_{o} Y^{\sharp}=2 \operatorname{deg} Y_{E}^{\sharp} \geqslant m ! K ! \mu .
$$

C другой стороны, имеем неравенство $\operatorname{deg} Z \geqslant \operatorname{mult}_{o} Z=\operatorname{deg} Z_{E}$ (справедливое для любого эффективного цикла $Z$ ).

Комбинируя эти оценки, получаем

$$
\begin{aligned}
m ! K ! n & \geqslant \operatorname{deg} Y=\operatorname{deg} Z+\operatorname{deg} Y^{\sharp} \\
& \geqslant \operatorname{deg} Z_{E}+2 \operatorname{deg} Y_{E}^{\sharp}=\operatorname{deg} Y_{E}+\operatorname{deg} Y_{E}^{\sharp} \\
& \geqslant \frac{1}{2} m ! K ! \nu+\frac{1}{2} m ! K ! \mu=\frac{1}{2} m ! K !(\nu+\mu) .
\end{aligned}
$$

Следовательно, $\nu+\mu \leqslant 2 n$. Противоречие.

Мы доказали, что центром максимальной особенности подвижной линейной системы не может быть особая точка слоя $o \in F$, лежащая на дивизоре ветвления морфизма $\sigma$.

Это завершает доказательство теоремы 1.6.

2.3. Послойные бирациональные перестройки. Докажем теорему 1.1 Пусть $V \in \mathscr{V}(d), F=V \cap\{p\} \times \mathbb{P}^{M}$ - слой над отмеченной точкой. Зафиксируем локальный параметр $t$ на кривой $C$ в окрестности точки $p$. Гиперповерхность $V \subset X$ в окрестности слоя $F$ задается уравнением

$$
f=f^{(0)}+t f^{(1)}+\cdots+t^{j} f^{(j)}+\cdots,
$$

где $f^{(j)}$ суть однородные многочлены степени $j$ от однородных координат $\left(x_{*}\right)=\left(x_{0}: \cdots: x_{M}\right)$ на $\mathbb{P}^{M}$. Хорошо известно [6], что

$$
\operatorname{dim} \operatorname{Sing} V \geqslant \operatorname{dim} \operatorname{Sing} F-1,
$$

так что из гладкости гиперповерхности $V$ вытекает, что, во-первых, гиперповерхность $F=\left\{f^{(0)}=0\right\} \subset \mathbb{P}^{M}$ имеет, самое большее, нульмерные особенности и, во-вторых, для каждой точки $x \in \operatorname{Sing} F$ имеем $f^{(1)}(x) \neq 0$.

Пусть $V_{1}, V_{2} \in \mathscr{V}(d)$ и $\chi^{*}: V_{1}^{*} \rightarrow V_{2}^{*}-$ послойный изоморфизм вне отмеченной точки $p \in C$. Поскольку слои над точками общего положения $y \in C$ суть гладкие гиперповерхности степени $d \geqslant 2$, над точкой $y \in C^{*}$ изоморфизм $\chi_{y}^{*}$ индуцирован автоморфизмом объемлющего проективного пространства $\xi_{y} \in \operatorname{Aut} \mathbb{P}$. Таким образом, $\chi^{*}=\left.\xi^{*}\right|_{V_{1}}$, где $\xi_{y}^{*}=\xi_{y}$ есть алгебраическая 
кривая $\xi^{*}: C^{*} \rightarrow$ Aut $\mathbb{P}$ проективных автоморфизмов. Пусть $\mathbb{P}=\mathbb{P}(L)$, где $L \cong \mathbb{C}^{M+1}$ - линейное пространство. Кривая $\xi^{*}$ может быть поднята до кривой $\xi: C \rightarrow$ End $L, \xi\left(C^{*}\right) \subset$ Aut $L$. Если $\xi(p) \in \operatorname{Aut} L$, то $\chi^{*}$ продолжается до послойного (бирегулярного) изоморфизма $\chi=\left.\xi\right|_{V_{1}}$, так что многообразия $V_{1}$ и $V_{2}$ послойно изоморфны. Предположим, что это не так: $\operatorname{det} \xi(p)=0$.

Пусть $\sum_{i=0}^{\infty} t^{i} \xi^{(i)}-$ ряд Тейлора кривой $\xi$. Можно считать, что $\xi^{(0)} \neq 0$. Следующее утверждение - это хорошо известный факт элементарной линейной алгебры.

Лемма 2.8. Существуют кривые эндоморфизмов $\beta, \gamma: C \rightarrow$ End $L$ и базис $\left(e_{0}, \ldots, e_{M}\right)$ пространства $L$ такие, что $\beta(p), \gamma(p) \in$ Aut $L$ и в этом базисе кривая $\beta \xi \gamma^{-1}: C \rightarrow$ End $L$ имеет диагональньй вид:

$$
\beta \xi \gamma^{-1}: e_{i} \mapsto t^{w\left(e_{i}\right)} e_{i}
$$

где $w\left(e_{i}\right) \in \mathbb{Z}_{+}$.

Заменим теперь $V_{1}$ на $\gamma\left(V_{1}\right)$ и $V_{2}$ на $\beta\left(V_{2}\right)$. Мы можем просто считать, что послойное бирациональное соответствие $\xi$ имеет вид (37) с самого начала. Покажем, что если $m=\max \left\{w\left(e_{i}\right)\right\} \geqslant 1$, то этого не может быть.

Пусть $\left\{a_{0}=0<a_{1}<\cdots<a_{k}\right\}=\left\{w\left(e_{i}\right), i=0, \ldots, M\right\} \subset \mathbb{Z}_{+}-$множество весов диагонального преобразования (37), $k \leqslant M, m=a_{k}$ - максимальный вес. Рассмотрим систему однородных координат $\left(x_{0}: \cdots: x_{M}\right)$, двойственную базису $\left(e_{*}\right)$. Определим вес мономов от $x_{*}$, полагая

$$
w\left(x_{0}^{n_{0}} x_{1}^{n_{1}} \cdots x_{M}^{n_{M}}\right)=\sum_{i=0}^{M} n_{i} w\left(e_{i}\right)
$$

Положим $\mathscr{A}_{i}=\left\{x_{j} \mid w\left(e_{j}\right)=a_{i}\right\} \subset \mathscr{A}=\left\{x_{0}, \ldots, x_{M}\right\}-$ множество координат веса $a_{i}$. Выделим особо множества $\mathscr{A}_{*}=\mathscr{A}_{0}$ и $\mathscr{A}^{*}=\mathscr{A}_{k}$ координат минимального и максимального веса соответственно.

Пусть теперь $f(x)=f^{(0)}(x)+t f^{(1)}(x)+\cdots$ - локальное по базе $C$ уравнение гиперповерхности $V_{2} \subset C \times \mathbb{P}, f^{(i)}$ - однородные многочлены степени $d \geqslant 3$ от координат $x_{*}$. Ряд

$$
f_{\xi}=\sum_{l=0}^{\infty} t^{l} f_{\xi}^{(l)}(x)=\sum_{l=0}^{\infty} t^{l} f^{(l)}\left(t^{w\left(x_{0}\right)} x_{0}, \ldots, t^{w\left(x_{M}\right)} x_{M}\right)
$$

обращается в нуль на $V_{1}$, а вне отмеченного слоя $F_{1}$, т. е. при $t \neq 0$, дает уравнение $V_{1}$. Пусть $b \in \mathbb{Z}_{+}-$максимальная степень параметра $t$, делящая $f_{\xi}$. Тогда

$$
t^{-b} f_{\xi}=g=\sum_{l=0}^{\infty} t^{l} g^{(l)}\left(x_{0}, \ldots, x_{M}\right)
$$

дает уравнение гиперповерхности $V_{1}$ и в отмеченном слое $X_{p}$.

ЛЕмма 2.9. Для любого $l \in \mathbb{Z}_{+}$многочлен $f^{(l)}$ есть линейная комбинация мономов веса $\geqslant b-l$, а многочлен $g^{(l)}$ есть линейная комбинация мономов веса $\leqslant b+l$. 
ДоКАЗАТЕЛЬСТво. Предположим, что моном $x^{I}$ входит в многочлен $f^{(l)}$ с ненулевым коэффициентом. Тогда он порождает компоненту $t^{l+w\left(x^{I}\right)} x^{I}$ ряда $f_{\xi}$ и, более того, эта компонента порождается толъко этим мономом в $f^{(l)}$. Следовательно, $l+w\left(x^{I}\right) \geqslant b$, как и утверждалось. Предположим, что моном $x^{I}$ входит в $g^{(l)}$ с ненулевым коэффициентом. Он происходит из монома $t^{l+b} x^{I}$ ряда $f_{\xi}$, который, в свою очередь, может происходить только из монома $x^{I}$ в составе многочлена $f^{(\alpha)}$, где $\alpha+w\left(x^{I}\right)=l+b$. Лемма доказана.

Пусть

$$
\begin{aligned}
& P_{*}=\left\{x_{j}=0 \mid w\left(x_{j}\right) \geqslant 1\right\}=\mathbb{P}\left\langle e_{j} \mid w\left(x_{j}\right)=0\right\rangle, \\
& P^{*}=\left\{x_{j}=0 \mid w\left(x_{j}\right) \leqslant m-1\right\}=\mathbb{P}\left\langle e_{j} \mid w\left(x_{j}\right)=m\right\rangle
\end{aligned}
$$

- подпространства минимального и максимального веса, соответственно.

Лемма 2.10. Если $b \geqslant m+1$, mо $P_{*} \subset \operatorname{Sing} F_{2}$. Ecли $m(d-1) \geqslant b+1, m o$ $P^{*} \subset \operatorname{Sing} F_{1}$.

ДоказатеЛЬСтво. Предположим, что $b \geqslant m+1$. Слой $F_{2} \subset \mathbb{P}$ над отмеченной точкой задается уравнением $f^{(0)}=0$. В силу предположения $f^{(0)}$ есть линейная комбинация мономов веса $\geqslant m+1$. Если моном $x^{I}$ входит в $f^{(0)}$ с ненулевым коэффициентом, то $x^{I}$ делится на квадратичный моном от переменных $\mathscr{A} \backslash \mathscr{A}_{*}$ (в противном случае $w\left(x^{I}\right) \leqslant m$ ). Поэтому все первые частные производные многочлена $f^{(0)}$ обращаются в нуль на $P_{*}$. Тем самым $P \subset \operatorname{Sing} F_{2}$.

Аналогично, если $b \leqslant m(d-1)-1$, то любой моном $x^{I}$ в $g^{(0)}$ делится на квадратичный моном от $\mathscr{A} \backslash \mathscr{A}^{*}$, в противном случае имеем $w\left(x^{I}\right) \geqslant m(d-1)$, что противоречит предположению и лемме 2.9. Лемма 2.10 доказана.

Заметим теперь, что при $d \geqslant 3$ неравенства $b \leqslant m$ и $b \geqslant m(d-1)$ не могут выполняться одновременно. Следовательно, выполнено хотя бы одно из двух неравенств леммы 2.10. Пусть $b \geqslant m+1$. Поскольку $V_{2}$ неособо, то $P_{*}-$ точка. Пусть $\mathscr{A}_{*}=\left\{x_{0}\right\}$, так что $P_{*}=(1,0, \ldots, 0)$. Снова в силу того, что $V_{2}$ неособо, имеем $f^{(1)}(1,0, \ldots, 0) \neq 0$. Следовательно, моном $x_{0}^{d}$ входит в $f^{(1)}$ с ненулевым коэффициентом. По лемме 2.9 получаем $b \leqslant 1$. Отсюда $m=0$ - противоречие.

В случае $b \leqslant m(d-1)-1$ рассуждения симметричны: $V_{1}$ неособо, $P^{*}$ - точка $(0, \ldots, 0,1), \mathscr{A}^{*}=\left\{x_{M}\right\}$ и $g^{(1)}(0, \ldots, 0,1) \neq 0$, так что $m d \leqslant b+1$, откуда снова получаем $m=0$ - противоречие.

Значит, нетривиальных весов быть не может, и $\xi$ - послойный бирегулярный изоморфизм. Следовательно, и $\chi=\left.\xi\right|_{V_{1}}$ есть послойный изоморфизм. Доказательство теоремы 1.1 закончено.

\section{§ 3. Многообразия с пучком кубических поверхностей}

В данном параграфе дается набросок доказательства теоремы 1.7 для $d=3$, т. е. для многообразий с пучком кубических поверхностей.

3.1. Максимальные особенности. Существование прямой. Пусть $\pi: V \rightarrow \mathbb{P}^{1}$ - расслоение на кубические поверхности, являющееся стандартным расслоением Фано, $\Sigma \subset\left|-n K_{V}+l F\right|-$ подвижная линейная система. Поскольку 
по предположению $V / \mathbb{P}^{1}$ удовлетворяет $K^{2}$-условию, выполнено и $K$-условие, т. е. $l \in \mathbb{Z}_{+}$. Теперь рассуждаем так же, как в $\S 1$, с единственным отличием: система $\Sigma$ может иметь максимальные особенности, накрывающие базу $\mathbb{P}^{1}$. Точнее, предположим, что имеет место неравенство $c_{\text {virt }}(\Sigma)<c(\Sigma, V)=n$. Тогда справедливо следующее предложение

ПредложениЕ 2.7. Линейная система $\Sigma$ обладает максимальной особенностью. Более того, либо найдется максимальная особенность, накрывающая базу $\mathbb{P}^{1}$, либо на некоторой модели $\varphi: V^{+} \rightarrow V$ имеется конечное множество исключительных дивизоров

$$
\mathscr{M}=\left\{E \subset V^{+} \mid e(E)=\operatorname{ord}_{E} \varphi^{*} \Sigma-n a(E)>0\right\}
$$

такое, что выполнено неравенство

$$
\sum_{t \in \mathbb{P}^{1}}\left(\max _{\left\{E \in \mathscr{M} \mid \varphi(E) \in F_{t}\right\}} \frac{e(E)}{\operatorname{ord}_{E} \varphi^{*} F_{t}}\right)>l .
$$

ДокАЗАТЕЛЬСтво стандартно (см. §1), и мы его не приводим.

Далее, если $C \subset V$ - горизонтальная кривая (т. е. $\pi(C)=\mathbb{P}^{1}$ ), являющаяся центром максимальной особенности, то $\operatorname{mult}_{C} \Sigma>n$, а потому $C$ - сечение или бисечение проекции $\pi$. Откручивая такие кривые послойными инволюциями, описанными в примере 1.4 главы 1, приходим к ситуации, когда система $\Sigma$ не имеет максимальных особенностей, накрывающих базу. Теперь, рассуждая как в $\S 1$, получаем существование сверхмаксимальной особенности $E \in \mathscr{M}$, удовлетворяющей неравенству

$$
6 n e(E)>\operatorname{ord}_{E} \varphi^{*} F_{t} \cdot \operatorname{deg} Z_{t}^{v},
$$

где $\varphi(E)=x \in F_{t}=\pi^{-1}(t)$ и $Z_{t}^{v}$ - вертикальная компонента самопересечения $Z=\left(D_{1} \circ D_{2}\right)$ линейной системы $\Sigma$, содержащаяся в слое $F_{t}$. Особенность $E$ с этого момента фиксирована, слой $F_{t}$ обозначается символом $F$, вместо $Z_{t}^{v}$ пишем $Z^{v}$ и т. д.

ПРЕДЛОЖЕНИЕ 2.8. Через точку $x \in F$ проходит хотя бъ одна прямая $L \subset F \subset \mathbb{P}^{3}$.

ДокАЗАтЕльство. Предположим противное. Тогда точка $x$ - неособая на кубической поверхности $F \subset \mathbb{P}^{3}$. Более того, кривая $R=T_{x} F \cap F$ неприводима, имеет степень 3 и кратность точно 2 в точке $x$. Если $C \subset F-$ любая другая кривая, то

$$
\operatorname{deg} C=(C \cdot R) \geqslant(C \cdot R)_{x} \geqslant 2 \operatorname{mult}_{x} C .
$$

Итак, для любой кривой $Q \subset F$ имеем

$$
\operatorname{mult}_{x} Q \leqslant \frac{2}{3} \operatorname{deg} Q .
$$

Следовательно,

$$
\text { mult }_{x} Z^{v} \leqslant \frac{2}{3} \operatorname{deg} Z^{v}<\frac{4 n e}{\nu_{E}(F)} .
$$

Теперь вычисления $\S 1$ (с упрощениями) дают противоречие. Предложение доказано. 
Основная трудность в доказательстве теоремы 1.7 при $d=3$ - это именно существование прямых, вклад которых в самопересечение $Z$ системы $\Sigma$ может быть слишком большим, так что вычисления $\S 1$ не дают противоречия. В настоящем обзоре рассмотрим случай, когда через точку $x$ на кубической поверхности $F$ проходит ровно одна прямая. Остальные случаи разобраны в [3].

Если прямая $L \ni x$ единственна, то точка $x$ неособа на $F$ и $T_{x} F \cap F=L+Q$, где $Q \subset F$ - неособая коника. Рассуждения, приведенные выше, показывают, что для любой кривой $C \subset F, C \neq L$, имеет место неравенство

$$
\operatorname{mult}_{x} C \leqslant \frac{1}{2} \operatorname{deg} C .
$$

Если записать $Z^{v}=C+k L$, где $C$ - эффективный 1-цикл, не содержащий $L$, то имеем:

$$
k+\frac{1}{2} \operatorname{deg} C \geqslant \frac{4 n e}{\nu_{E}(F)}, \quad k+\operatorname{deg} C<\frac{6 n e}{\nu_{E}(F)} .
$$

Отсюда следует, что

$$
\operatorname{deg} C<\frac{4 n e}{\nu_{E}(F)} .
$$

В частности, $(C \cdot L) \leqslant \operatorname{deg} C<\frac{4 n e}{\nu_{E}(F)}$.

3.2. Основная конструкция: лестница, связанная с прямой $L$. Предполагаем, что прямая $L$ не содержит особых точек слоя $F$ (если таковые имеются). Общий случай разобран в [3].

Бесконечную последовательность раздутий

$$
\begin{aligned}
\sigma_{i}: & V^{(i)} \longrightarrow V^{(i-1)} \\
\cup & \cup \\
E^{(i)} & \longrightarrow L_{i-1}
\end{aligned}
$$

$i \geqslant 1$, начинающуюся с $V^{(0)}=V$, где $L_{i-1}$ - центр $i$-го раздутия, а $E^{(i)}=$ $\sigma_{i}^{-1}\left(L_{i-1}\right)$ - исключительный дивизор, $L_{0}=L$, назовем лестницей, ассоциированной с прямой $L$, если выполнены следующие условия:

1) $L_{i}-$ кривая для всех $i \in \mathbb{Z}_{+}$,

2) $E^{(i)}$ - линейчатая поверхность типа $\mathbb{F}_{1}$ над $L_{i-1}$,

3) $L_{i} \subset E^{(i)}$ - исключительное сечение (т. е. (-1)-кривая).

Очевидно, что этим описанием лестница определяется однозначно. Чуть ниже будет показано, что она существует. Ее начальный отрезок, состоящий из раздутий $\sigma_{i}$ для $1 \leqslant i \leqslant M$, будем называть (конечной) лестницей длины $M$.

Доказательство существования лестницы удобно провести одновременно с доказательством некоторых ее свойств.

Для удобства обозначений пусть $E^{(0)}=F$ - слой морфизма $\pi$, содержащий прямую $L$. Операцию взятия собственного прообраза на $i$-й ступеньке (т. е. на $V^{(i)}$ ) обозначаем добавлением верхнего индекса $i$ в скобках. Например, собственный прообраз поверхности $E^{(i)}$ на $V^{(j)}$ при $j \geqslant i$ записывается как $E^{(i, j)}$. Пусть также:

$s_{i}-$ класс $L_{i}$ в $A^{2}\left(V^{(i)}\right), s_{0}=f ;$

$f_{i} \in A^{2}\left(V^{(i)}\right)$ - класс слоя линейчатой поверхности $E^{(i)}$ над точкой $\in L_{i-1}$. 
Злоупотребляя обозначениями, мы будем понимать иногда $s_{i}$ и $f_{i}$ как численные классы кривых на линейчатой поверхности $E^{(i)}$ :

$$
A^{1} E^{(i)}=\operatorname{Pic} E^{(i)}=\mathbb{Z} s_{i} \oplus \mathbb{Z} f_{i},
$$

так что, в частности, осмысленны записи вида $\left(s_{i} \cdot s_{i}\right)=-1,\left(s_{i} \cdot f_{i}\right)=1$ и т. д.

В этих обозначениях имеет место следующее предложение.

ПредлОЖениЕ 2.9. (i) При $i \geqslant 1$ эбфективный 1-uикл $\left(E^{(i-1, i)} \circ E^{(i)}\right)$ есть просто неприводимая кривая $E^{(i-1, i)} \cap E^{(i)}$, численный класс которой есть $\left(s_{i}+f_{i}\right)$. В частности, эта кривая не пересекается с $L_{i} \sim s_{i}$.

(ii) Справедливы равенства $\left(E^{(i)}\right)^{3}=1,\left(E^{(i)} \cdot L_{i}\right)=0$. С учетом изоморфизма $L_{i} \cong \mathbb{P}^{1}$ имеет место изоморфизм пучков

$$
\mathscr{N}_{L_{i} / V^{(i)}} \cong \mathscr{O}_{L_{i}} \oplus \mathscr{O}_{L_{i}}(-1) .
$$

Если однозначно определенная первая компонента в этом представлении соответствует исключительному сечению $L_{i+1} \subset E^{(i+1)}=\mathbb{P}\left(\mathscr{N}_{L_{i} / V^{(i)}}\right)$, то в качестве второй компоненты можно взять одномерное подрасслоение, соответствующее как раз кривой $E^{(i, i+1)} \cap E^{(i+1)}$.

(iii) Классы $s_{i} u f_{i}$ удовлетворяют соотношениям

$$
\sigma^{*} s_{i-1}=s_{i}, \quad \sigma_{*} f_{i}=0
$$

npu $i \geqslant 1$.

ДокАЗАТЕльство. Приведем одновременно доказательство существования лестницы и предложения 2.9. По предположению, напомним, $L \cap \operatorname{Sing} F=\varnothing$.

Рассмотрим первый шаг лестницы - морфизм $\sigma_{1}: V^{(1)} \rightarrow V^{(0)}=V$, раздувающий прямую $L_{0}=L \subset F$. Имеем точную последовательность

$$
\left.0 \rightarrow \mathscr{N}_{L / F} \rightarrow \mathscr{N}_{L / V} \rightarrow \mathscr{O}_{V}(F)\right|_{L} \rightarrow 0
$$

которая может быть переписана в виде

$$
0 \rightarrow \mathscr{O}_{L}(-1) \rightarrow \mathscr{N}_{L / V} \rightarrow \mathscr{O}_{L} \rightarrow 0
$$

Следовательно, $E^{(1)}$ - линейчатая поверхность типа $\mathbb{F}_{1},\left(E^{(1)}\right)^{3}=1$, откуда $\left(E^{(1)} \cdot E^{(1)}\right) \sim\left(-s_{1}-f_{1}\right)$ и $\left(E^{(0,1)} \cdot L_{1}\right)=\left(\left(F-E^{(1)}\right) \cdot s_{1}\right)=0$. Тем самым, все требования определения лестницы для первого раздутия выполнены.

Далее доказательство проводится индукцией по $i \geqslant 1$. Имеем точную последовательность

$$
\left.0 \rightarrow \mathscr{N}_{L_{i} / E^{(i)}} \rightarrow \mathscr{N}_{L_{i} / V^{(i)}} \rightarrow \mathscr{O}_{V^{(i)}}\left(E^{(i)}\right)\right|_{L_{i}} \rightarrow 0 .
$$

С учетом доказанного она может быть переписана в виде

$$
0 \rightarrow \mathscr{O}_{L_{i}}(-1) \rightarrow \mathscr{N}_{L_{i} / V^{(i)}} \rightarrow \mathscr{O}_{L_{i}} \rightarrow 0
$$

Снова отсюда следует, что $E^{(i+1)}=\mathbb{P}\left(\mathscr{N}_{L_{i} / V^{(i)}}\right)$ есть линейчатая поверхность типа $\mathbb{F}_{1}$ и $\left(E^{(i+1)}\right)^{3}=1$, так что

$$
\left.E^{(i+1)}\right|_{E^{(i+1)}} \sim\left(-s_{i+1}-f_{i+1}\right) .
$$

Поэтому $\left(E^{(i+1)} \cdot L_{i+1}\right)=0$. Утверждения (i) и (iii) выполнены очевидным образом. Доказательство завершено. 
ЗАмЕчАнИЕ 2.2. (i) Поскольку $E^{(i-1, i)}$ не пересекается с $L_{i}$ при $i \geqslant 1$, имеем

$$
E^{(i-1, i)}=E^{(i-1, i+1)}=\cdots=E^{(i-1, j)}=\cdots
$$

для любого $j \geqslant i$. В частности, если $C \subset E^{(i-1)}-$ кривая, отличная от исключительного сечения $L_{i-1}$, то ее собственный прообраз на всех $V^{(j)}, j \geqslant i$, один и тот же:

$$
C^{(i)}=C^{(i+1)}=\cdots=C^{(j)}
$$

(ii) Злоупотребляя терминологией, назовем неприводимую кривую $C \subset E^{(i)}$, $i \geqslant 1$, горизонтальной, если $\sigma_{i}(C)=L_{i-1}$, и вертикальной, если $\sigma_{i}(C)$ - точка на $L_{i-1}$. Соответственно определим горизонтальные и вертикальные 1-циклы, носитель которых содержится в $E^{(i)}$. Степень горизонтальной кривой $C$ есть $\operatorname{deg} C=\left.\operatorname{deg} \sigma_{i}\right|_{C}=\left(C \cdot f_{i}\right)$, степень вертикальной кривой $C$ есть $\operatorname{deg} C=$ $\left(C \cdot L_{i}\right)=1$. Степень горизонтального и вертикального 1-цикла с носителем в $E^{(i)}$ определим как пересечение с $f_{i}$ и $L_{i}$ соответственно. В частности, степень вертикального 1-цикла есть просто количество входящих в него прямых-слоев. Отметим, что если эффективный горизонтальный 1-цикл $C$ не содержит в качестве компоненты исключительное сечение $L_{i}$, то его класс в $A^{1}\left(E^{(i)}\right)$ или $A^{2}\left(V^{(i)}\right)$ есть $\alpha s_{i}+\beta f_{i}$, где $\alpha \geqslant 1$ и $\beta \geqslant \alpha$.

(iii) Очевидно, что граф последовательности раздутий $\sigma_{i}$ есть цепь. В частности,

$$
K_{V^{(M)}}=\sigma_{M, 0}^{*} K_{V}+\sum_{i=1}^{M} \sigma_{M, i}^{*} E^{(i)}
$$

(где $\sigma_{i, j}$, как всегда, обозначает композицию $\sigma_{j+1} \circ \cdots \circ \sigma_{i}$ ) и дискрепантность нормирования $\nu_{E^{(i)}}$ есть $i$. Далее,

$$
\sigma_{M, 0}^{*} F=F^{(M)}+\sum_{i=1}^{M} E^{(i, M)} .
$$

ПРЕДЛОЖЕНИЕ 2.10. Существует конечная $L$-лестница длины $M \geqslant 1$ maкая, что для $i=0, \ldots, M-1$ иентр нормирования $\nu_{E}$ на $V^{(i)}$ есть точка $x_{i} \in L_{i}, x_{0}=x$, в то время как для центра $\nu_{E}$ на $V^{(M)}$ реализуется одна из следуюших возможностей:

A) $x_{M} \notin L_{M}, x_{M} \notin E^{(M-1, M)}$;

В) прямая $B=\sigma_{M}^{-1}\left(x_{M-1}\right)$ - слой линейчатой поверхности $E^{(M)}$;

C) $x_{M}=E^{(M-1, M)} \cap \sigma_{M}^{-1}\left(x_{M-1}\right)$.

ДокАЗАтЕЛьство. Если центр нормирования $\nu_{E}$ на $V^{(i)}$ содержится в $E^{(i)}$, то $i=a\left(E^{(i)}, V\right) \leqslant a\left(\nu_{E}, V\right)$. Следовательно, существует такое $M \geqslant 1$, что центр нормирования $\nu_{E}$ на $V^{(M)}$ не есть точка на $L_{M}$. Оставшаяся часть доказательства - очевидный перебор возможностей. Предложение доказано.

Зафиксируем построенную лестницу длины $M$. 
3.3. Техника подсчета кратностей. Мы ограничимся исключением случая А): случаи В) и С) исключаются аналогично, но формулы несколько более громоздки. См. [3] по поводу всех деталей. Пусть $\Sigma^{(i)}-$ собственный прообраз системы $\Sigma$ на $V^{(i)}$. Положим $\lambda_{i}=\operatorname{mult}_{L_{i-1}} \Sigma^{(i-1)}, n \geqslant \lambda_{1} \geqslant \cdots$. Поверхность $E^{(M)} \subset V^{(M)}$ обозначим для удобства символом $E^{*}$. Пусть

$$
\begin{aligned}
\varphi_{i, i-1}: & V_{i} \longrightarrow V_{i-1} \\
& \cup \quad \cup \quad i=1, \ldots, K, \quad V_{0}=V^{(M)}, \\
& E_{i} \longrightarrow B_{i-1}
\end{aligned}
$$

- разрешение нормирования $\nu_{E}$, рассматриваемого как дискретное нормирование на многообразии $V^{(M)}$. Введем ряд обозначений:

$\nu_{i}=\operatorname{mult}_{B_{i-1}} \Sigma^{i-1}-$ кратность собственного прообраза системы $\Sigma$ на $V_{i-1}$ вдоль раздуваемого цикла;

$p_{i}=p\left(E_{K}, E_{i}\right)$ - число путей в ориентированном графе нормирования $\nu_{E}=\nu_{E_{K}}$ из $E_{K}$ в $E_{i}$ (здесь $\nu_{E}$ рассматривается как дискретное нормирование на многообразии $\left.V_{0}=V^{(M)} !\right)$;

$N^{*}=\max \left\{i \mid 1 \leqslant i \leqslant K, B_{i-1} \subset E^{i-1}\right\} ;$

$L=\max \left\{i \mid 1 \leqslant i \leqslant K, B_{i-1}-\right.$ точка $\}$ (так что $B_{j-1}-$ точка при $j \leqslant L$ и кривая при $j \geqslant L+1)$; наконец, положим

$$
N=\min \left\{N^{*}, L\right\}, \quad \Sigma_{0}=\sum_{i=1}^{L} p_{i}, \quad \Sigma_{1}=\sum_{i=L+1}^{K} p_{i}, \quad \Sigma^{*}=\sum_{i=1}^{N^{*}} p_{i}, \quad \Sigma_{*}=\sum_{i=1}^{N} p_{i} .
$$

Очевидно, что в этих обозначениях имеем $\nu_{E}\left(E^{*}\right)=\varepsilon=\Sigma^{*}$ и $\nu_{E}(F)=\varepsilon$. Далее,

$$
\nu_{E}(\Sigma)=\nu_{E^{*}}(\Sigma) \nu_{E}\left(E^{*}\right)+\nu_{E}\left(\Sigma^{(M)}\right) .
$$

Теперь неравенство Нётера-Фано принимает вид

$$
\sum_{i=1}^{K} p_{i} \nu_{i}=\varepsilon \sum_{i=1}^{M}\left(n-\lambda_{i}\right)+n \sum_{i=1}^{K} p_{i} \delta_{i}+e .
$$

Как всегда, пусть $D_{i}^{(M)}, i=1,2,-$ собственные прообразы общих дивизоров из системы $\Sigma$. Пусть $Z^{(M)}=\left(D_{1}^{(M)} \circ D_{2}^{(M)}\right)$ - эффективный 1-цикл их теоретико-схемного пересечения. Положим

$$
m_{i}=\operatorname{mult}_{B_{i-1}}\left(Z^{(M)}\right)^{i-1}
$$

для $i \leqslant L$. Согласно технике подсчета кратностей, имеем оценку

$$
\sum_{i=1}^{L} p_{i} m_{i} \geqslant \frac{\left(2 \Sigma_{0} n+\Sigma_{1} n+\varepsilon \sum_{i=1}^{M}\left(n-\lambda_{i}\right)+e\right)^{2}}{\Sigma_{0}+\Sigma_{1}} .
$$

Оценивая минимум этой квадратичной формы при указанных ограничениях и с учетом неравенства Нётера-Фано, получаем

$$
\operatorname{mult}_{B} Z^{(M)} \geqslant \frac{\left(\sum n+\varepsilon \sum_{i=1}^{M}\left(n-\lambda_{i}\right)+e\right)^{2}}{\sum_{i=1}^{K} p_{i}^{2}} .
$$


3.4. Цикл $Z^{(M)}$ в терминах лестницы. Теперь для того чтобы завершить доказательство теоремы 1.7, необходимо оценить сверху левую часть только что полученного основного неравенства. Соответствующие выкладки довольно утомительны, хотя геометрически вполне прозрачны. Возвращаясь к нашей основной конструкции - лестнице, - введем ряд новых понятий и обозначений, связанных с линейной системой $\Sigma$. Прежде всего, пусть

$$
z_{i}=\left(D_{1}^{(i)} \cdot D_{2}^{(i)}\right) \in A^{2} V^{(i)}
$$

- класс эффективного 1-цикла $Z^{(i)}=\left(D_{1}^{(i)} \circ D_{2}^{(i)}\right)$. На “нулевом" этаже нашей лестницы мы имеем разложение

$$
Z=Z^{v}+Z^{h}
$$

Проследим, как меняется 1-цикл $Z^{(k)}$ при переходе от $k=i-1$ к $k=i$. Естественно, компоненты цикла $Z^{(i-1)}$, отличные от $L_{i-1}$, присутствуют в $Z^{(i)}$ уже своими собственными прообразами. Вместо кривой $L_{i-1}$, входящей в $Z^{(i-1)}$ с некоторой кратностью $k_{i-1}$, цикл $Z^{(i)}$ содержит эффективный подцикл, носитель которого содержится в исключительном дивизоре $E^{(i)}$. Разобьем этот подцикл на три части:

1) часть $C_{h}^{(i)}$ включает все кривые, горизонтальные относительно морфизма $\sigma_{i}: E^{(i)} \rightarrow L_{i-1}$ и отличные от исключительного сечения $L_{i}$;

$2)$ часть $C_{v}^{(i)}$ включает все вертикальные кривые, т. е. слои $\sigma_{i}$ над точками кривой $L_{i-1}$;

$3)$ исключительное сечение $L_{i}$ с некоторой кратностью $k_{i} \in \mathbb{Z}_{+}$.

Для единообразия обозначений положим: $C_{h}^{(0)}-$ часть цикла $Z^{v}$, отличная от прямой $L$. Положим также

$$
d_{h, v}^{(i)}=\operatorname{deg} C_{h, v}^{(i)}
$$

(см. замечание 2.2 , (ii)). Теперь имеем следующее представление циклов $Z^{(i)}$ :

$$
\begin{aligned}
& Z^{(0)}=Z^{h}+Z^{v}=Z^{h}+C_{h}^{(0)}+k_{0} L, \\
& Z^{(1)}=\left(Z^{h}\right)^{(1)}+C_{h}^{(0,1)}+C_{h}^{(1)}+C_{v}^{(1)}+k_{1} L_{1}, \\
& Z^{(i)}=\left(Z^{h}\right)^{(i)}+C_{h}^{(0,1)}+C_{h}^{(1,2)}+C_{v}^{(1,2)}+\cdots \\
& +C_{h}^{(i-1, i)}+C_{v}^{(i-1, i)}+C_{h}^{(i)}+C_{v}^{(i)}+k_{i} L_{i} .
\end{aligned}
$$

3.5. Подсчет класса $z_{M}$ и завершение доказательства теоремы 1.7. Очевидно, что класс цикла $C_{v}^{(i)}$ в $A^{1} V^{(i)}$ есть $d_{v}^{(i)} f_{i}$, а класс цикла $C_{h}^{(i)}$ есть $d_{h}^{(i)} s_{i}+\beta_{i} f_{i}$, где коэффициенты удовлетворяют важному неравенству

$$
\beta_{i} \geqslant d_{h}^{(i)}
$$

(см. замечание 2.2 , (ii)). Далее, класс цикла $C_{v}^{(i, i+1)}$ есть $d_{v}^{(i)}\left(f_{i}-f_{i+1}\right)$, а класс цикла $C_{h}^{(i, i+1)}$ есть

$$
d_{h}^{(i)} s_{i}+\beta_{i} f_{i}-\left(\beta_{i}-d_{h}^{(i)}\right) f_{i+1}
$$


Полагая $\alpha_{i}=\left(\left(Z^{h}\right)^{(i-1)} \cdot L_{i-1}\right)$, можем записать $z_{i}^{h}=z_{i-1}^{h}-\alpha_{i} f_{i}$, где $z_{i}^{h}$ есть численный класс горизонтального цикла $\left(Z^{h}\right)^{(i)}$.

Лемма 2.11. Имеет место неравенство

$$
\alpha_{i} \leqslant \operatorname{deg} Z^{h}=3 n^{2} .
$$

ДокАЗАТЕЛЬСтво. Поскольку $L \subset F$, a $\operatorname{deg} Z^{h}$ есть $\left(Z^{h} \cdot F\right)$, это очевидно.

ПРЕДЛОЖЕНИЕ 2.11. Классъ $z_{i}$ удовлетворяют иепочке соотношений

$$
z_{i}=z_{i-1}-\left(2 \lambda_{i} n+\lambda_{i}^{2}\right) f_{i}-\lambda_{i}^{2} s_{i}
$$

ДокАзАтельство. Имеем

$$
\begin{aligned}
z_{i} & =\left(D^{(i)}\right)^{2}=\left(D^{(i-1)}-\lambda_{i} E^{(i)}\right)^{2} \\
& =z_{i-1}-2 \lambda_{i}\left(D^{(i-1)} \cdot L_{i-1}\right) f_{i}-\lambda_{i}^{2}\left(s_{i}+f_{i}\right) .
\end{aligned}
$$

Из доказанного выше следует, что для любого $\left(D^{(j)} \cdot L_{j}\right)=(D \cdot L)=n j \in \mathbb{Z}_{+}$, что и требовалось.

ПредлОЖЕНИЕ 2.12. При $i \geqslant 2$ целъе числа $k_{i}, \alpha_{i}, \beta_{i} u d_{h, v}^{(i)}$ удовлетворяют системе соотношений

$$
d_{v}^{(i)}+\beta_{i}=\alpha_{i}+d_{v}^{(i-1)}+\left(\beta_{i-1}-d_{h}^{(i-1)}\right)-2 \lambda_{i} n-\lambda_{i}^{2} .
$$

При $i=1$ имеем $d_{v}^{(1)}+\beta_{1}=\alpha_{1}+\left(C_{h}^{(0)} \cdot L\right)-2 \lambda_{1} n-\lambda_{1}^{2}$.

ДокАЗАтЕльство. Необходимо явно расписать класс цикла $Z^{(i)}$ в терминах введенных нами параметров и воспользоваться предыдущим предложением. Соответствующие выкладки элементарны.

ПРЕДЛОЖЕНИЕ 2.13. При любом $i \geqslant 1$ имеет место неравенство

$$
d_{v}^{(i)}+\beta_{i} \leqslant\left(C_{h}^{(0)} \cdot L\right)+\sum_{j=1}^{i}\left(3 n^{2}-2 \lambda_{j} n-\lambda_{j}^{2}\right) .
$$

ДокАЗАТЕЛЬСтво. Необходимо $i$ раз применить предложение 2.12 и воспользоваться леммой 2.11 .

Завершим, наконец, исключение случая А). Ясно, что из всех кривых, содержащихся в дивизоре

$$
\bigcup_{i=0}^{M} E^{(i, M)}
$$

точку $x_{M}$ могут содержать только те, которые целиком лежат в $E^{(M)}$ и отличны от исключительного сечения $L_{M}$. Значит, мы вправе написать

$$
Z^{(M)}=\left(Z^{h}\right)^{(M)}+C_{v}^{(M)}+C_{h}^{(M)}+\cdots,
$$

где многоточие обозначает сумму кривых, не содержащих точку $x_{M}$. Положим

$$
W=C_{v}^{(M)}+C_{h}^{(M)}, \quad m_{i}^{v}=\operatorname{mult}_{B_{i-1}} W^{i-1}, \quad m_{i}^{h}=\operatorname{mult}_{B_{i-1}}\left(Z^{h}\right)^{(M), i-1}
$$


для $i \leqslant L$, так что $m_{i}=m_{i}^{v}+m_{i}^{h}$. Очевидно, что кратности $m_{i}^{v}$ обращаются в нуль при $N+1 \leqslant i \leqslant L$. Далее, $m_{i}^{h, v} \leqslant m_{1}^{h, v}$, и, как и в лемме 2.11 , имеем $m_{1}^{h} \leqslant 3 n^{2}$. Наконец, $m_{1}^{v} \leqslant d_{v}^{(M)}+d_{h}^{(M)} \leqslant d_{v}^{(M)}+\beta_{M}$, так что в итоге получаем:

$$
\begin{aligned}
3 n^{2} \Sigma_{0}+\Sigma_{*}\left(\left(C_{h}^{(0)} \cdot L\right)+\sum_{i=1}^{M}\left(3 n^{2}-2 \lambda_{i} n-\lambda_{i}^{2}\right)\right) \\
\geqslant \sum_{i=1}^{L} p_{i} m_{i}^{h}+\sum_{i=1}^{N} p_{i} m_{i}^{v} \geqslant \frac{\left(2 \Sigma_{0} n+\Sigma_{1} n+\varepsilon \sum_{i=1}^{M}\left(n-\lambda_{i}\right)+e\right)^{2}}{\Sigma_{0}+\Sigma_{1}} .
\end{aligned}
$$

Если заменить $\Sigma_{*}$ на $\varepsilon=\Sigma^{*}$, неравенство может лишь усилиться, а если заменить $\varepsilon\left(C_{h}^{(0)} \cdot L\right)$ на $4 n e$, то оно станет строгим. Перенесем все в правую часть и рассмотрим полученное выражение как квадратичную форму от $\lambda_{i}$ на области $0 \leqslant \lambda_{i} \leqslant n$. В силу симметрии, ее минимум достигается на диагонали, т. е. при $\lambda_{i}=\lambda, 0 \leqslant \lambda \leqslant n$. Заменим все $\lambda_{i}$ на это значение $\lambda$, при этом строгое неравенство сохранится. В итоге получаем

$$
\Phi<0
$$

где выражение $\Phi$ может быть с помощью элементарной арифметики преобразовано к виду

$$
\begin{gathered}
\Phi=\left(\Sigma_{0}^{2}+\Sigma_{0} \Sigma_{1}+\Sigma_{1}^{2}\right) n^{2}+M \varepsilon \Sigma_{0}(n-\lambda)^{2}-M \varepsilon \Sigma_{1}(n-\lambda)(n+\lambda) \\
+M^{2} \varepsilon^{2}(n-\lambda)^{2}-2 e \Sigma_{1} n+2 M \varepsilon e(n-\lambda)+e^{2} .
\end{gathered}
$$

Поскольку $\lambda \leqslant n$, можно заменить $(n+\lambda)$ на $2 n$ с сохранением строгого неравенства. Нетрудно, однако, проверить, что последнее выражение есть сумма полного квадрата

$$
\left(\Sigma_{1} n-M \varepsilon(n-\lambda)-e\right)^{2}
$$

и нескольких неотрицательных слагаемых. Поэтому оно не может быть отрицательным, что и завершает доказательство теоремы 1.7.

\section{Глава 3}

\section{Многообразия, имеющие много рационально связных структур}

\section{§ 1. Прямые произведения Фано}

В данном параграфе, следуя [33], приведено доказательство теоремы 1.9 о бирациональной сверхжесткости прямых произведений Фано и доказана дивизориальная каноничность общих гиперповерхностей Фано индекса единица.

1.1. Максимальные особенности подвижных линейных систем. Доказательство теоремы 1.9 проводится индукцией по числу сомножителей $K$. При $K=1$ теорема справедлива тривиальным образом: условие (M) означает, что подвижные линейные системы на многообразии $F=F_{1}$ не имеют максимальных особенностей. Отсюда сразу следует бирациональная сверхжесткость многообразия $F$. 
Начиная с этого момента, $K \geqslant 2$.

Предположим противное: на $V$ имеется подвижная линейная система $\Sigma$, для которой справедливо неравенство $c_{\text {virt }}(\Sigma)<c(\Sigma)$. В силу определения виртуального порога канонического присоединения это означает, что существует последовательность раздутий $\varphi: \widetilde{V} \rightarrow V$ такая, что выполнено неравенство

$$
c(\widetilde{\Sigma})<c(\Sigma)
$$

где $\widetilde{\Sigma}$ - собственный прообраз линейной системы $\Sigma$ на $\widetilde{V}$. Для доказательства бирациональной сверхжесткости многообразия $V$ необходимо установить, что неравенство (38) невозможно, т. е. получить противоречие.

Пусть $H_{i}=-K_{F_{i}}-$ положительная образующая группы Pic $F_{i}$. Положим

$$
S_{i}=\prod_{j \neq i} F_{j}
$$

так что $V \cong F_{i} \times S_{i}$. Пусть $\rho_{i}: V \rightarrow F_{i}$ и $\pi_{i}: V \rightarrow S_{i}$ - проекции на сомножители. Злоупотребляя обозначениями, пишем $H_{i}$ вместо $\rho_{i}^{*} H_{i}$, так что

$$
\operatorname{Pic} V=\bigoplus_{i=1}^{K} \mathbb{Z} H_{i} \quad \text { и } \quad K_{V}=-H_{1}-\cdots-H_{K} .
$$

Имеем $\Sigma \subset\left|n_{1} H_{1}+\cdots+n_{K} H_{K}\right|$, причем $c(\Sigma)=\min \left\{n_{1}, \ldots, n_{K}\right\}$. Без ограничения общности считаем, что $c(\Sigma)=n_{1}$. В силу неравенства $(38)$ имеем $n_{1} \geqslant 1$. Положим $n=n_{1}, \pi=\pi_{1}, F=F_{1}, S=S_{1}$. Получаем

$$
\Sigma \subset\left|-n K_{V}+\pi^{*} Y\right|
$$

где $Y=\sum_{i=2}^{K}\left(n_{i}-n\right) H_{i}-$ эффективный класс на базе $S$ расслоения $\pi$.

Теперь необходимо модифицировать бирациональный морфизм $\varphi$. Для произвольной последовательности раздутий $\mu_{S}: S^{+} \rightarrow S$ полагаем $V^{+}=F \times S^{+}$ и получаем коммутативную диаграмму

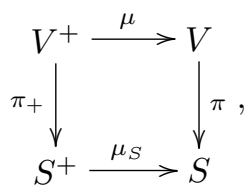

где $\pi_{+}-$проекция, $\mu=\left(\operatorname{id}_{F}, \mu_{S}\right)$. Пусть $E_{1}, \ldots, E_{N} \subset \widetilde{V}$ - все исключительные дивизоры морфизма $\varphi$.

ПредЛОжЕНИЕ 3.1. Существует такая последовательность раздутий $\mu_{S}: S^{+} \rightarrow S$, что в обозначениях диаграммы (39) иентр любого дискретного нормирования $E_{i}, i=1, \ldots, N$, накрывает либо $S^{+}$, либо некоторый дивизор на $S^{+}$:

$$
\operatorname{codim}\left[\pi_{+}\left(\operatorname{centre}\left(E_{i}, V^{+}\right)\right)\right] \leqslant 1 .
$$


ДокАЗАТЕЛЬСтво. Пусть $E \subset \widetilde{V}$ - исключительный дивизор бирационального морфизма $\varphi: \widetilde{V} \rightarrow V$, а $B=\varphi(E)$ - центр дискретного нормирования $E$ на $V$. Предположим, что $\operatorname{codim}_{S} \pi(B) \geqslant 2$. Построим последовательность коммутативных диаграмм

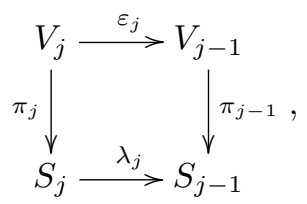

$j=1, \ldots, l$, удовлетворяющую следующим требованиям:

1) $V_{0}=V, S_{0}=S, \pi_{0}=\pi$

2) $V_{j}=F \times S_{j}, \pi_{j}$ есть проекция на сомножитель $S_{j}, \varepsilon_{j}=\left(\operatorname{id}_{F}, \lambda_{j}\right)$ для всех $j \geqslant 1$;

3) $\lambda_{j}$ есть раздутие неприводимого многообразия

$$
B_{j-1}=\pi_{j-1}\left(\operatorname{centre}\left(E, V_{j-1}\right)\right) \subset S_{j-1},
$$

где $\operatorname{codim} B_{j-1} \geqslant 2$.

Очевидно, что свойства 1)-3) однозначно определяют последовательность диаграмм (40).

Лемма 3.1. Имеет место неравенство $l \leqslant a(E, V)$.

ДоказАтельство. Пусть $\Delta_{j} \subset V_{j}$ - исключительный дивизор морфизма $\varepsilon_{j}$. По построению имеем $\operatorname{centre}\left(E, V_{j}\right) \subset \Delta_{j}$, стало быть, $\nu_{E}\left(\Delta_{j}\right) \geqslant 1$. Теперь получаем

$$
a(E, V)=a\left(E, V_{l}\right)+\sum_{j=1}^{l} \nu_{E}\left(\Delta_{j}\right) a\left(\Delta_{j}, V\right) \geqslant l .
$$

Лемма доказана.

Поэтому последовательность диаграмм (40) обрывается: можно считать, что $\operatorname{centre}\left(E, V_{l}\right)$ накрывает дивизор на базе $S_{l}$. Отсюда (в силу теоремы Хиронаки о разрешении особенностей) немедленно вытекает предложение 3.1.

Пусть $\Sigma^{+}-$собственный прообраз линейной системы $\Sigma$ на $V^{+}$. Дальнейшие рассуждения разбиваются на две части в силу следующего факта.

ПрЕДЛОЖЕНИЕ 3.2. Имеет место альтернатива:

(i) либо выполнено неравенство $c\left(\Sigma^{+}\right)<c(\Sigma)$,

(ii) либо для общего дивизора $D^{+} \in \Sigma^{+}$nара $\left(V^{+}, n^{-1} D^{+}\right)$не канонична, причем для некоторого $i=1, \ldots, N$ дискретное нормирование $E_{i}$ реализует неканоническую особенность этой пары.

ЗАмечАниЕ 3.1. Альтернативу предложения 3.2 следует понимать в том смысле, что по менъшей мере одна из двух возможностей (i), (ii) имеет место (возможно, обе). 
ДоКАЗАТЕЛЬСТВо ПРЕДЛОЖЕНИЯ 3.2. Рассмотрим диаграмму отображений (39). Пусть $\tau: V^{\sharp} \rightarrow V^{+}$- разрешение особенностей сквозного отображения

$$
V^{+} \stackrel{\mu}{\longrightarrow} V \stackrel{\varphi^{-1}}{\longrightarrow} \widetilde{V}
$$

Положим $\psi=\varphi^{-1} \circ \mu \circ \tau: V^{\sharp} \rightarrow \widetilde{V}$. Это бирациональный морфизм неособых проективных многообразий. Существуют открытое множество $U \subset V^{\sharp}$ и замкнутое множество коразмерности два $Y \subset \widetilde{V}$ такие, что

$$
\psi_{U}=\left.\psi\right|_{U}: U \rightarrow \widetilde{V} \backslash Y
$$

есть изоморфизм. Очевидно, если $E \subset V^{\sharp}-$ исключительный дивизор морфизма $\tau$ и $E \cap U \neq \varnothing$, то $E \cap U=\psi_{U}^{-1}\left(E_{i}\right)$ для некоторого исключительного дивизора $E_{i}$ морфизма $\varphi$.

Пусть $\Sigma^{+}$и $\Sigma^{\sharp}-$ собственные прообразы линейной системы $\Sigma$ на $V^{+}$и $V^{\sharp}$ соответственно, $\Sigma_{U}=\left.\Sigma^{\sharp}\right|_{U}$. Если $D^{\sharp} \in \Sigma^{\sharp}-$ общий дивизор, то

$$
\widetilde{D}=\psi_{U}\left(D_{U}^{\sharp}\right) \in \widetilde{\Sigma}
$$

- общий дивизор линейной системы $\widetilde{\Sigma}$ (мы не различаем $\widetilde{\Sigma}$ и ее ограничение на $\widetilde{V} \backslash Y$, потому что $Y$ имеет коразмерность два). Мы знаем, что

$$
\widetilde{D}+n K_{\widetilde{V}} \notin A_{+}^{1} \widetilde{V}
$$

(см. (38)). Следовательно,

$$
D_{U}^{\sharp}+n K_{U} \notin A_{+}^{1} U
$$

Пусть $\mathscr{E}$ - множество исключительных дивизоров морфизма $\tau$, имеющих непустое пересечение с $U$. В силу (41) получаем

$$
\left.\tau^{*}\left(D^{+}+n K^{+}\right)\right|_{U}-\sum_{E \in \mathscr{E}}\left(\nu_{E}\left(D^{+}\right)-n a^{+}(E)\right) E_{U} \notin A_{+}^{1} U
$$

где $K^{+}$- канонический класс многообразия $V^{+}, a^{+}(E)=a\left(E, V^{+}\right)$. Следовательно, либо

$$
D^{+}+n K^{+} \notin A_{+}^{1} V^{+}
$$

и тогда имеет место альтернатива (i) предложения 3.2, либо существует исключительный дивизор $E \in \mathscr{E}$, удовлетворяющий неравенству Нётера-Фано $\nu_{E}\left(D^{+}\right)>n a^{+}(E)$, т. е. дискретное нормирование $E$ реализует неканоническую особенность пары $\left(V^{+}, n^{-1} D^{+}\right)$. В последнем случае имеет место альтернатива (ii) предложения 3.2 , так как $E \in \mathscr{E}$ и потому $E=E_{i}$ для некоторого $i=1, \ldots, N$ (как дискретные нормирования). Этим предложение 3.2 доказано.

1.2. Редукция к базе расслоения. Предположим, что имеет место альтернатива (i) предложения 3.2 , т. е. $D^{+}+n K^{+} \notin A_{+}^{1} V^{+}$. Пусть $z \in F$ - точка общего положения. Положим

$$
S_{z}^{+}=\{z\} \times S^{+}, \quad S_{z}=\{z\} \times S .
$$


Понятно, что $K_{z}^{+}=\left.K^{+}\right|_{S_{z}}$ и $K_{z}=\left.K_{V}\right|_{S_{z}}$ суть канонические классы $K_{S}^{+}$и $K_{S}$ соответственно. Пусть

$$
\Sigma_{z}=\left.\Sigma\right|_{S_{z}}, \quad \Sigma_{z}^{+}=\left.\Sigma^{+}\right|_{S_{z}^{+}}
$$

- ограничение линейных систем $\Sigma, \Sigma^{+}$на $S_{z}, S_{z}^{+}$, и пусть $D_{z} \in \Sigma_{z}$ и $D_{z}^{+} \in \Sigma_{z}^{+}-$ общие дивизоры. Получаем подвижную линейную систему $\Sigma_{z}$ на многообразии $S=F_{2} \times \cdots \times F_{K}$, причем

$$
\Sigma_{z} \subset\left|n_{2} H_{2}+\cdots+n_{K} H_{K}\right|,
$$

так что $c\left(\Sigma_{z}\right)=\min \left\{n_{2}, \ldots, n_{K}\right\} \geqslant n=c(\Sigma)$.

Лемма 3.2. Имеет место равенство $D^{+}+n K^{+}=\pi_{+}^{*}\left(D_{z}^{+}+n K_{z}^{+}\right)$.

ДокАЗАТЕЛьСтво. Пусть $\mathscr{E}_{S}-$ множество исключительных дивизоров морфизма $\mu_{S}$. Исключительные дивизоры морфизма $\mu$ суть $F \times E=\pi_{+}^{*} E$ для $E \in \mathscr{E}_{S}$. Имеем

$$
K_{S}^{+}=\mu_{S}^{*} K_{S}+\sum_{E \in \mathscr{E}_{S}} a_{E} E \quad \text { и } \quad K^{+}=\mu^{*} K_{V}+\pi_{+}^{*}\left(\sum_{E \in \mathscr{E}_{S}} a_{E} E\right),
$$

где $a_{E}=a(E)$ - дискрепантность дивизора $E$. Для некоторых чисел $b_{E} \geqslant 0$ имеем

$$
D^{+}=\mu^{*} D-\sum_{E \in \mathscr{E}_{S}} b_{E} \pi_{+}^{*} E,
$$

причем для точки $z \in F$ общего положения

$$
D_{z}^{+}=\mu_{S}^{*} D_{z}-\sum_{E \in \mathscr{E}_{S}} b_{E} E
$$

Теперь, учитывая, что $D+n K_{V}=\pi^{*} Y$ и что $D_{z}+n K_{z}=D_{z}+n K_{S}=Y$, получаем утверждение леммы.

СлеДСТвиЕ 3.1. $D_{z}^{+}+n K_{z}^{+} \notin A_{+}^{1} S^{+}$.

ДоказАТЕЛьство. В самом деле, очевидно, что

$$
\pi_{+}^{*} A_{+}^{1} S^{+} \subset A_{+}^{1} V^{+},
$$

что и доказывает следствие.

Таким образом, для собственного прообраза $\Sigma_{z}^{+}$линейной системы $\Sigma_{z}$ на $S^{+}$ получаем неравенство $c\left(\Sigma_{z}^{+}\right)<c\left(\Sigma_{z}\right)$. Тем более, $c_{\text {virt }}\left(\Sigma_{z}\right)<c\left(\Sigma_{z}\right)$. Значит, многообразие $S$ не является бирационально сверхжестким. Противоречие с предположением индукции.

1.3. Редукция к слою расслоения. Завершение доказательства бирациональной сверхжесткости многообразия $V$. В силу предложения 3.2 и сказанного выше, для общего дивизора $D^{+} \in \Sigma^{+}$пара $\left(V^{+}, n^{-1} D^{+}\right)$не канонична, т. е. имеется исключительный дивизор $E \subset V^{\sharp}$ некоторого бирационального морфизма $V^{\sharp} \rightarrow V^{+}$, удовлетворяющий неравенству Нётера-Фано 
$\nu_{E}\left(\Sigma^{+}\right)>n a_{E}^{+}$, где $a_{E}^{+}=a\left(E, V^{+}\right)$. Более того, можно предполагать, что центр $B=\operatorname{centre}\left(E, V^{+}\right)$нормирования $E$ накрывает дивизор на базе или всю базу: $\operatorname{codim}_{S^{+}} T \leqslant 1$, где $T=\pi_{+}(B)$.

Пусть $t \in T$ - точка общего положения. Слой $F_{t}=\pi_{+}^{-1}(t)$ не может целиком содержаться в базисном множестве Bs $\Sigma^{+}$подвижной системы $\Sigma^{+}$, так как

$$
\operatorname{codim}_{V^{+}} \pi_{+}^{-1}(T) \leqslant 1
$$

Следовательно, $\Sigma_{t}^{+}=\left.\Sigma^{+}\right|_{F_{t}}-$ непустая линейная система на $F, \Sigma_{t}^{+} \subset|n H|=$ $\left|-n K_{F}\right|$ (если $T \subset S^{+}$- дивизор, то $\Sigma_{t}^{+}$может иметь неподвижные компоненты). Пусть $D_{t}^{+} \in \Sigma_{t}^{+}-$общий дивизор. Согласно принципу обращения присоединения (теорема 1.8 и 22 настоящей главы), пара

$$
\left(F, \frac{1}{n} D_{t}^{+}\right)
$$

не логканонична. Получаем противоречие с условием (L). Это противоречие завершает доказательство бирациональной сверхжесткости многообразия $V$.

\section{4. Структуры рационально связного расслоения и бирациональ-} ные автоморфизмы. Докажем остальные утверждения теоремы 1.9. Пусть $\beta: V^{\sharp} \rightarrow S^{\sharp}-$ рационально связное расслоение, $\chi: V \rightarrow V^{\sharp}-$ бирациональное отображение. Возьмем очень обильную линейную систему $\Sigma_{S}^{\sharp}$ на базе $S^{\sharp}$, и пусть $\Sigma^{\sharp}=\beta^{*} \Sigma_{S}^{\sharp}-$ подвижная линейная система на $V^{\sharp}, c\left(\Sigma^{\sharp}\right)=0$. Пусть $\Sigma-$ собственный прообраз системы $\Sigma^{\sharp}$ на $V$. В силу сказанного $c_{\text {virt }}(\Sigma)=0$, так что по доказанному имеем $c(\Sigma)=0$. Следовательно, в представлении

$$
\Sigma \subset\left|-n_{1} H_{1}-\cdots-n_{K} H_{K}\right|
$$

найдется коэффициент $n_{e}$ равный нулю. Для определенности считаем, что $e=1$. Пусть $S=F_{2} \times \cdots \times F_{K}, \pi: V \rightarrow S$ - проекция, тогда $\Sigma \subset\left|\pi^{*} Y\right|$ для некоторого неотрицательного класса $Y$ на $S$. Но это означает, что бирациональное отображение $\chi$ расслоения $V / S$ в расслоение $V^{\sharp} / S^{\sharp}$ является послойным: существует рациональное доминантное отображение $\gamma: S \rightarrow S^{\sharp}$, обеспечивающее коммутативность диаграммы

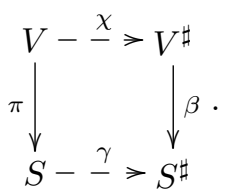

Для точки общего положения $z \in S^{\sharp}$ пусть $F_{z}^{\sharp}=\beta^{-1}(z)-$ соответствующий слой, $F_{z}^{\chi} \subset V$ - его собственный прообраз относительно $\chi$. По предположению, многообразие $F_{z}^{\chi}$ рационально связно. С другой стороны,

$$
F_{z}^{\chi}=\pi^{-1}\left(\gamma^{-1}(z)\right)=F \times \gamma^{-1}(z),
$$

где $F=F_{1}-$ слой $\pi$. Следовательно, слой $\gamma^{-1}(z)$ также рационально связен. 
Таким образом, задача описания рационально связных структур на $V$ сводится к той же самой задаче на $S$. Теперь утверждение (i) теоремы 1.9 легко получается индукцией по числу прямых сомножителей $K$. (Для $K=1$ отсутствие нетривиальных рационально связных структур очевидно.) Вторая часть утверждения (i) (о структурах расслоения на коники и рациональные поверхности) очевидна в силу того, что $\operatorname{dim} F_{i} \geqslant 3$ для всех $i=1, \ldots, K$. Нерациональность многообразия $V$ теперь очевидна.

Докажем утверждение (ii) теоремы 1.9. Пусть $R C(V)$ - множество структур рационально связного расслоения с нетривиальной базой на многообразии $V$. Согласно утверждению (i), имеем

$$
R C(V)=\left\{\pi_{I}: V \rightarrow F_{I}=\prod_{i \in I} F_{i} \mid \varnothing \neq I \subset\{1, \ldots, K\}\right\} .
$$

На множестве $R C(V)$ имеется естественное отношение порядка: $\alpha \leqslant \beta$, если $\beta$ пропускается через $\alpha$. Очевидно, что $\pi_{I} \leqslant \pi_{J}$, если и только если $J \subset I$. Для $I=\{1, \ldots, K\} \backslash\{e\}$ положим $\pi_{I}=\pi_{e}, F_{I}=S_{e}$. Очевидно, что $\pi_{1}, \ldots, \pi_{K}$ суть минимальные элементы $R C(V)$.

Пусть $\chi \in \operatorname{Bir} V$ - бирациональный автоморфизм. Имеем биекцию

$$
\begin{aligned}
& \chi^{*}: R C(V) \rightarrow R C(V), \\
& \chi^{*}: \alpha \mapsto \alpha \circ \chi,
\end{aligned}
$$

причем $\chi^{*}$ сохраняет отношение $\leqslant$. Отсюда легко вывести, что $\chi^{*}$ имеет вид

$$
\chi^{*}: \pi_{I} \mapsto \pi_{I^{\sigma}},
$$

где $\sigma \in S_{K}$ - некоторая перестановка $K$ элементов и $I^{\sigma}=\left\{\sigma\left(i_{1}\right), \ldots, \sigma\left(i_{k}\right)\right\}$ для $I=\left\{i_{1}, \ldots, i_{k}\right\}$. Далее, для каждого $I \subset\{1, \ldots, K\}$ имеем диаграмму

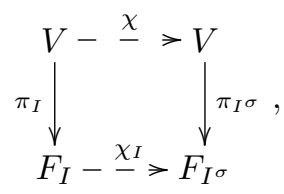

где $\chi_{I}$ - некоторое бирациональное отображение. В частности, $\chi$ индуцирует бирациональные изоморфизмы $\chi_{e}: F_{e} \rightarrow F_{\sigma(e)}, e=1, \ldots, K$. Однако все многообразия $F_{e}$ бирационально сверхжесткие, так что все отображения $\chi_{e}$ суть изоморфизмы. Стало быть, и

$$
\chi=\left(\chi_{1}, \ldots, \chi_{K}\right) \in \operatorname{Bir} V
$$

есть бирегулярный изоморфизм, $\chi \in \operatorname{Aut} V$.

Теорема 1.9 полностью доказана.

1.5. Пример многообразий, удовлетворяющих условию дивизориальной каноничности. Пусть $\mathbb{P}=\mathbb{P}^{M}, M \geqslant 3$, - комплексное проективное пространство. Положим $\mathscr{F}=\mathbb{P}\left(H^{0}\left(\mathbb{P}, \mathscr{O}_{\mathbb{P}}(M)\right)\right)$ - пространство гиперповерхностей степени $M$. 
Теорема 3.1. При $M \geqslant 6$ существует непустое открытое по Зарискому подмножество $\mathscr{F}_{\text {reg }} \subset \mathscr{F}$ такое, что для любого уравнения $f \in \mathscr{F}_{\text {reg }}$ coответствующая гиперповерхность $F=\{f=0\}$ неособа и удовлетворяет условию $(\mathrm{C})$.

ДокАЗАТЕЛЬство. Подмножество $\mathscr{F}_{\text {reg }}$ задается явными условиями регулярности, которые мы сейчас сформулируем. Пусть $F=F_{M} \subset \mathbb{P}=\mathbb{P}^{M}$ гладкая гиперповерхность Фано. В точке $x \in F$ зафиксируем систему аффинных координат $z_{1}, \ldots, z_{M}$ с началом в точке $x$. Пусть

$$
f=q_{1}+\cdots+q_{M}
$$

- уравнение гиперповерхности $F, q_{i}=q_{i}\left(z_{*}\right)$ - однородные многочлены степени $\operatorname{deg} q_{i}=i$,

$$
f_{i}=q_{1}+\cdots+q_{i}
$$

- левые сегменты многочлена $f, i=1, \ldots, M$.

Условие (R1.1). Последовательность

$$
q_{1}, \ldots, q_{M-1}
$$

регулярна в кольце $\mathscr{O}_{x, \mathbb{P}}$, т. е., система уравнений

$$
q_{1}=\cdots=q_{M-1}=0
$$

задает одномерное подмножество - конечный набор прямых в $\mathbb{P}$, проходящих через точку $x$.

Это стандартное условие, использованное в [1; гл. 3].

Условие (R1.2). Линейная оболочка любой неприводимой компоненты замкнутого алгебраического множества

$$
q_{1}=q_{2}=q_{3}=0
$$

в $\mathbb{C}^{M}$ есть гиперплоскость $q_{1}=0$ (т. е. касательная гиперплоскость $T_{x} F$ ).

Условие (R1.3). Замкнутое алгебраическое множество

$$
\overline{\left\{f_{1}=f_{2}=0\right\} \cap F}=\overline{\left\{q_{1}=q_{2}=0\right\} \cap F} \subset \mathbb{P}
$$

(черта сверху означает замыкание в $\mathbb{P}$ ) неприводимо и любое сечение этого множества некоторой гиперплоскостью $P \ni x$

(i) либо также неприводимо и приведено,

(ii) либо распадается на две неприводимые компоненты $B_{1}+B_{2}$, где $B_{i}=$ $F \cap S_{i}$ есть сечение $F$ плоскостью $S_{i} \subset \mathbb{P}$ коразмерности 3 , и, более того, $\operatorname{mult}_{x} B_{i}=3$,

(iii) либо неприведено и имеет вид $2 B$, где $B=F \cap S$ есть сечение $F$ плоскостью $S$ коразмерности 3 , и, более того, mult $_{x} B=3$.

Пусть $\mathscr{F}_{\text {reg }} \subset \mathscr{F}-$ множество гиперповерхностей Фано, удовлетворяющих условиям (R1.1)-(R1.3) в каждой точке (в частности, любая гиперповерхность $F \in \mathscr{F}_{\text {reg }}$ гладкая). Понятно, что $\mathscr{F}_{\text {reg }}$ - открытое по Зарискому подмножество проективного пространства $\mathscr{F}$. Имеет место следующее предложение

ПреДЛОЖЕНИЕ 3.3. При $M \geqslant 6$ множество $\mathscr{F}$ reg неnусто. 
ДокАЗАТЕЛЬСтво дано в [33]. При $M \geqslant 8$ в условии (R1.3) можно потребовать, чтобы сечение множества (42) любой гиперплоскостью $P \ni x$ было неприводимо и приведено. С другой стороны, при $M=4,5$ легко показать, что для любой гиперповерхности $F \in \mathscr{F}$ найдется точка, в которой условия (R1.2) и (R1.3) не выполнены.

Докажем справедливость условия $(\mathrm{C})$ для регулярной гиперповерхности Фано $F \in \mathscr{F}_{\text {reg }}$.

Пусть $\Delta \in|n H|$ - эффективный дивизор, $n \geqslant 1$, где $H \in \operatorname{Pic} F-$ класс гиперплоского сечения, $K_{F}=-H$. Необходимо показать, что пара $\left(F, n^{-1} \Delta\right)$ имеет канонические особенности.

Предположим противное. Тогда для некоторой последовательности раздутий $\varphi: F^{+} \rightarrow F$ и исключительного дивизора $E^{+} \subset F^{+}$выполнено неравенство Нётера-Фано

$$
\nu_{E^{+}}(\Delta)>n a\left(E^{+}\right) .
$$

При фиксированном $E^{+}$неравенство (43) линейно по $\Delta$, так что без ущерба для общности можно считать, что $\Delta \subset F$ - простой дивизор, т. е. неприводимое подмногообразие коразмерности 1. Из (43) легко следует, что центр $Y=\varphi\left(E^{+}\right)$ нормирования $E^{+}$на $F$ удовлетворяет неравенству mult $Y>n$. С другой стороны, хорошо известно [1; лемма 2.1], что для любой неприводимой кривой $C \subset F$ имеет место неравенство $\operatorname{mult}_{C} \Delta \leqslant n$. Поэтому $Y=x-$ точка. Пусть $\varepsilon: \widetilde{F} \rightarrow F$ - ее раздутие, $E \subset \widetilde{F}$ - исключительный дивизор $E \cong \mathbb{P}^{M-2}$. Согласно доказываемому ниже предложению 3.6 для некоторой гиперплоскости $B \subset E$ выполнено неравенство

$$
\operatorname{mult}_{x} \Delta+\operatorname{mult}_{B} \widetilde{\Delta}>2 n,
$$

где $\widetilde{\Delta} \subset \widetilde{F}-$ собственный прообраз дивизора $\Delta$.

Пусть $\mathbb{T}=\overline{T_{x} F} \subset \mathbb{P}$ - касательная гиперплоскость в точке $x$. Дивизор $E$ естественно отождествляется с проективизацией $\mathbb{P}\left(T_{x} \mathbb{T}\right)=\mathbb{P}\left(T_{x} F\right)$. Существует единственная гиперплоскость $\mathbb{B} \subset \mathbb{T}, x \in \mathbb{B}$, такая, что $B=\mathbb{P}\left(T_{x} \mathbb{B}\right)$ относительно указанного выше отождествления. Пусть $\Lambda_{\mathbb{B}}-$ пучок гиперплоскостей в $\mathbb{P}$, содержащих $\mathbb{B}$, и $\Lambda_{B}=\left.\Lambda_{\mathbb{B}}\right|_{F} \subset|H|$ - его ограничение на $F$. Рассмотрим общий дивизор $R \in \Lambda_{B}$. Это гиперповерхность степени $M$ в $\mathbb{P}^{M-1}$, гладкая в точке $x$. Пусть $\widetilde{R} \subset \widetilde{F}-$ собственный прообраз дивизора $R$. Очевидно, что

$$
\widetilde{R} \cap E=B .
$$

Положим $\Delta_{R}=\left.\Delta\right|_{R}=\Delta \cap R$. Это эффективный дивизор на гиперповерхности $R$.

Лемма 3.3. Имеет место оценка

$$
\operatorname{mult}_{x} \Delta_{R}>2 n \text {. }
$$

ДоказАТЕЛЬСтво. Имеем $(\widetilde{\Delta} \circ \widetilde{R})=\widetilde{\Delta}_{R}+Z$, где $Z$ - эффективный дивизор на $E$. В соответствии с правилами элементарной теории пересечений [44], $\operatorname{mult}_{x} \Delta_{R}=\operatorname{mult}_{x} \Delta+\operatorname{deg} Z$, поскольку $\operatorname{mult}_{x} R=1$. Однако $Z$ содержит $B$ с кратностью по меньшей мере $\operatorname{mult}_{B} \widetilde{\Delta}$. Поэтому из неравенства (44) вытекает оценка (45). Лемма доказана. 
Лемма 3.4. Дивизор $T_{R}=T_{x} R \cap R$ на гиперповерхности $R$ неприводим и имеет кратность точно 2 в точке $x$.

ДоказАтельство. Неприводимость очевидна (например, при $M \geqslant 6$ можно применить теорему Лефшеца). Согласно условию (R1.2), квадрика $\left\{\left.q_{2}\right|_{E}=0\right\}$ не содержит в качестве компоненты гиперплоскость в $E$, в частности, она не содержит гиперплоскость $B \subset E$. Поэтому квадратичная компонента уравнения дивизора $T_{R}$, т. е. многочлен $\left.q_{2}\right|_{B}$, отличен от нуля. Лемма доказана.

Продолжим доказательство теоремы 3.1. В силу лемм 3.3 и 3.4 можно записать

$$
\Delta_{R}=a T_{R}+\Delta_{R}^{\sharp},
$$

где $a \in \mathbb{Z}_{+}$и эффективный дивизор $\Delta_{R}^{\sharp} \in\left|n^{\sharp} H_{R}\right|$ на гиперповерхности $R$ удовлетворяет оценке $\operatorname{mult}_{x} \Delta_{R}^{\sharp}>2 n^{\sharp}$. Более того, $\Delta_{R}^{\sharp}$ не содержит дивизор $T_{R}$ в качестве компоненты. Без ущерба для общности можно считать, что дивизор $\Delta_{R}^{\sharp}$ неприводим и приведен.

Теперь рассмотрим вторую гиперкасательную систему [1; гл. 3]

$$
\Lambda_{2}^{R}=\left|s_{0} f_{2}+s_{1} f_{1}\right|_{R}
$$

где $s_{i}$ - однородные многочлены степени $i$ от линейных координат $z_{*}$. Ее базисное множество

$$
S_{R}=\left\{\left.q_{1}\right|_{R}=\left.q_{2}\right|_{R}=0\right\}
$$

имеет в силу условия (R1.3) коразмерность 2 в $R$ и либо неприводимо и имеет кратность 6 в точке $x$, либо распадается на два плоских сечения гиперповерхности $R$, каждое кратности 3 в точке $x$. В любом случае для общего дивизора $D \in \Lambda_{2}^{R}$ получаем: $\Delta_{R}^{\sharp} \not \subset \operatorname{Supp} D$, так что на $R$ корректно определен эффективный цикл

$$
\Delta_{D}=\left(D \circ \Delta_{R}^{\sharp}\right)
$$

коразмерности два. Поскольку mult $x=3$ и $\Lambda_{2}^{R} \subset\left|2 H_{R}\right|$, цикл $\Delta_{D}$ удовлетворяет оценке

$$
\frac{\operatorname{mult}_{x}}{\operatorname{deg}} \Delta_{D}>\frac{3}{M} .
$$

Заменяя цикл $\Delta_{D}$ его подходящей неприводимой компонентой, можно предполагать его неприводимым подмногообразием коразмерности 2 в $R$. Сравнивая оценку (46) с данным выше описанием множества $S_{R}$, видим, что $\Delta_{D} \not \subset S_{R}$. Отсюда вытекает, что $\Delta_{D} \not \subset T_{R}$. В самом деле, если бы это было не так, мы имели бы

$$
\left.\left.f_{1}\right|_{\Delta_{D}} \equiv q_{1}\right|_{\Delta_{D}} \equiv 0 .
$$

Однако $\Delta_{D} \subset D$, так что для некоторых $s_{0} \neq 0, s_{1} \neq 0$ (дивизор $D$ выбран достаточно общим) имеем

$$
\left.\left(s_{0} f_{2}+s_{1} f_{1}\right)\right|_{\Delta_{D}} \equiv 0 .
$$

Согласно (47), отсюда следует, что $\left.\left.f_{2}\right|_{\Delta_{D}} \equiv\left(q_{1}+q_{2}\right)\right|_{\Delta_{D}} \equiv 0$ (поскольку $s_{0} \neq 0$ есть просто константа), так что $\Delta_{D} \subset S_{R}$. Противоречие. 
Итак, $\Delta_{D} \not \subset T_{R}$. Следовательно, эффективный цикл $\Delta^{+}=\left(\Delta_{D} \circ T_{R}\right)$ корректно определен и удовлетворяет оценке

$$
\frac{\operatorname{mult}_{x}}{\operatorname{deg}} \Delta^{+}>\frac{6}{M} .
$$

Эффективный цикл $\Delta^{+}$имеет коразмерность 4 как цикл на $F$. Напомним теперь следующий факт (см. [45] или [1; гл. 3]): если гиперповерхность Фано $F$ в точке $x$ удовлетворяет условию регулярности (R1.1), то для любого эффективного цикла $Y$ чистой коразмерности $l \leqslant M-2$ выполнено неравенство

$$
\frac{\operatorname{mult}_{x}}{\operatorname{deg}} Y \leqslant \frac{l+2}{M} .
$$

Следовательно, неравенство (48) для эффективного цикла коразмерности 4 невозможно.

Этим противоречием теорема 3.1 доказана.

\section{§ 2. Принцип связности и его приложения}

В данном параграфе приведен принцип связности Шокурова-Коллара и рассмотрены его геометрические приложения.

2.1. Принцип связности. Обращение присоединения. Пусть $X, Z$ нормальные многообразия или аналитические пространства и $h: X \rightarrow Z$ - собственный морфизм со связными слоями, а $D=\sum d_{i} D_{i}-\mathbb{Q}$-дивизор на $X$.

Теорема 3.2 (принцип связности [41; теорема 17.4]). Предположим, что дивизор $D$ эфбективен $\left(d_{i} \geqslant 0\right)$, а класс $-\left(K_{X}+D\right)$ является $h$-численно эффективным и

$$
f: Y \stackrel{h}{\longrightarrow} X \stackrel{h}{\longrightarrow} Z
$$

- разрешение особенностей пары $(X, D)$. Положим

$$
K_{Y}=g^{*}\left(K_{X}+D\right)+\sum e_{i} E_{i}
$$

Тогда носитель $\mathbb{Q}$-дивизора $\sum_{e_{i} \leqslant-1} e_{i} E_{i}$, m.е. замкнутое алгебраическое множе-
ство

$$
\bigcup_{e_{i} \leqslant-1} E_{i},
$$

является связным в окрестности любого слоя морфизма $f$.

Доказательство см. в [41; гл. 17]. Оно также воспроизведено в обзоре [15] и в [46] для частного случая (в котором рассуждения следуют той же схеме, что и в [41]).

Принцип связности имеет многочисленные приложения, которые мы сейчас рассмотрим. Первое приложение - это теорема 1.8 (обращение присоединения). 
ДоКАЗАТЕЛЬСТВО ТЕОРЕМЫ 1.8. Пользуемся обозначениями теоремы 1.8 . Пусть $D=\sum_{i \in I} d_{i} D_{i}$ - эффективный $\mathbb{Q}$-дивизор, $d_{i} \in \mathbb{Q}_{+}$для всех $i \in I$. Поскольку пара $(X, D)$ канонична вне точки $x$, имеем неравенство $d_{i} \leqslant 1$ для всех $i \in I$. Заменяя $D$ на $(1+\varepsilon)^{-1} D$ для малого $\varepsilon \in \mathbb{Q}_{+}$, можно считать, что $d_{i}<1$ для всех $i \in I$.

Пусть $\varphi: \widetilde{X} \rightarrow X-$ разрешение особенностей пары $(X, D+R)$. Запишем

$$
K_{\widetilde{X}}=\varphi^{*}\left(K_{X}+D+R\right)+\sum_{j \in J} e_{j} E_{j}-\sum_{i \in I} d_{i} \widetilde{D}_{i}-\widetilde{R}
$$

где $E_{j}, j \in J,-$ все исключительные дивизоры морфизма $\varphi$, а $\widetilde{D}_{i}$ и $\widetilde{R}$ - собственные прообразы дивизоров $D_{i}, R$ на $\widetilde{X}$ соответственно. Положим

$$
b_{j}=\operatorname{ord}_{E_{j}} \varphi^{*} D, \quad a_{j}=a\left(E_{j}, X\right),
$$

$j \in J$. В этих обозначениях для $j \in J$ имеем $e_{j}=a_{j}-b_{j}-r_{j}$, где $r_{j}=\operatorname{ord}_{E_{j}} \varphi^{*} R$. Очевидно, что

$$
\varphi^{-1}(x)=\bigcup_{j \in J^{+}} E_{j}
$$

для некоторого подмножества $J^{+} \subset J$. Напомним, что по предположению $R-$ дивизор Картье, содержащий точку $x$. Отсюда следует (это ключевой момент), что для $j \in J^{+}$

$$
r_{j}=\operatorname{ord}_{E_{j}} \varphi^{*} R \geqslant 1
$$

Далее, по предположению пара $(X, D)$ не канонична, но канонична вне точки $x$. Следовательно, среди $j \in J^{+}$найдется такой индекс $k$, что $a_{k}<b_{k}$. Для этого индекса имеем $e_{k}<-1$.

В силу принципа связности найдется такой индекс $l \in J$, что $e_{l}<-1$ и

$$
E_{l} \cap \widetilde{R} \neq \varnothing .
$$

Теперь из (49) по формуле присоединения получаем

$$
K_{\widetilde{R}}=\left.\left(K_{\widetilde{X}}+\widetilde{R}\right)\right|_{\widetilde{R}}=\varphi_{R}^{*}\left(K_{R}+D_{R}\right)+\left(\left.\sum_{j \in J} e_{j} E_{j}\right|_{\widetilde{R}}-\left.\sum_{i \in I} d_{i} \widetilde{D}_{i}\right|_{\widetilde{R}}\right),
$$

где $\varphi_{R}=\left.\varphi\right|_{\widetilde{R}}: \widetilde{R} \rightarrow R$ есть ограничение последовательностей раздутий $\varphi$ на $R$. В силу сказанного в последней скобке найдется хотя бы один простой дивизор вида $\left.E_{l}\right|_{\widetilde{R}}$, где $l \in J^{+}$, коэффициент при котором строго меньше -1 . Теорема 1.8 доказана.

Полезен следующий вариант обращения присоединения.

ПРЕДЛОЖЕНИЕ 3.4. Пусть $x \in X$ - росток гладкого многообразия, $D$ эффективный $\mathbb{Q}$-дивизор, пара $(X, D)$ не канонична, но канонична вне точки $x$, m.е. точка $x$ - изолированный центр неканонических особенностей этой nары. Пусть $R \ni x$-неособый дивизор, причем $T_{x} R$ - гиперплоскость общего положения в $T_{x} X$. Тогда пара $\left(R, D_{R}\right)$ не логканонична, но канонична вне точки $x$. 
ДокАЗАтЕльство. В обозначениях доказательства теоремы 1.8 , индекс $k$, реализующий не логканоническую особенность пары $\left(R, D_{R}\right)$, лежит в $J^{+}:$в силу условия общности положения, дивизор $R$ не содержит центров особенностей пары $(X, D)$ вне точки $x$. Этим предложение доказано.

Вот еще одно следствие принципа связности.

ПреДЛОЖЕНИЕ 3.5. Пусть $x \in X$ - росток гладкого многообразия, $D$ эфбективныи $\mathbb{Q}$-дивизор, пара $(X, D)$ не канонична в точке $x$, но канонична вне этой точки. Пусть $\lambda: X^{+} \rightarrow X-$ раздутие точки $x, E=\lambda^{-1}(x) \subset X^{+}-$ исключительный дивизор, $D^{+}$- собственный прообраз дивизора D. Далее, пусть $\mu: \widetilde{X} \rightarrow X^{+}-$разрешение особенностей пары $\left(X^{+}, D^{+}\right)$,

$$
\varphi=\lambda \circ \mu: \tilde{X} \rightarrow X
$$

- сквозное отображение. Запишем

$$
K_{\widetilde{X}}=\varphi^{*}\left(K_{X}+D\right)+\sum_{j \in J} e_{j} E_{j}-\sum_{i \in I} d_{i} \widetilde{D}_{i},
$$

где $E_{j}, j \in J,-$ все исключительные дивизоры морфизма $\varphi$, а $\widetilde{D}_{i}$ - собственные прообразы дивизоров $D_{i}$ на $\widetilde{X}$. Тогда имеет место следующая альтернатива:

1) либо $\operatorname{mult}_{x} D>\operatorname{dim} X$,

2) либо множество

$$
\mu\left(\bigcup_{b_{j}>a_{j}+1} E_{j}\right) \subset E
$$

связно.

ДокАЗАТЕЛЬСтво. Это немедленно вытекает из принципа связности.

2.2. Дальнейшие приложения принципа связности. Прежде всего, установим следующий полезный факт.

ПреДЛОЖеНИЕ 3.6. Пусть пара $(X, D)$ - такая же, как в предложении 3.5 , $\lambda: X^{+} \rightarrow X$ - раздутие точки $x, E=\lambda^{-1}(x) \subset X^{+}-$исключительный дивизор, $D^{+}-$собственный прообраз дивизора $D$. Тогда имеет место следующая альтернатива:

1) либо $\operatorname{mult}_{x} D>2$,

2) либо найдется гиперплоскость $B \subset E$, однозначно определяемая парой $(X, D)$, такая, что выполнено неравенство

$$
\operatorname{mult}_{x} D+\operatorname{mult}_{B} D^{+}>2 .
$$

ДоказАтеЛьство. Каноничность сильнее логканоничности. Поэтому обращение присоединения (в форме предложения 3.4) можно применять многократно, последовательно ограничивая пару $(X, D)$ на гладкие подмногообразия

$$
R_{1} \supset R_{2} \supset \cdots \supset R_{k},
$$

$R_{1} \subset X-$ гладкий дивизор, $R_{i+1} \subset R_{i}$ - гладкий дивизор, $x \in R_{k}$ и $R_{k} \not \subset$ Supp D. Все пары

$$
\left(R_{i},\left.D\right|_{R_{i}}\right)
$$


не логканоничны в точке $x$. Поэтому предложение 3.4 справедливо для любого гладкого ростка $R \ni x$ коразмерности $k \leqslant \operatorname{dim} X-1$. В частности, оно справедливо для общей поверхности $S \ni x$. Итак, пара $\left(S, D_{S}=\left.D\right|_{S}\right)$ имеет в точке $x$ изолированную (для общей поверхности $S$ ) не логканоническую особенность. Рассмотрим подробнее двумерную ситуацию. Имеем: $x \in S$-росток гладкой поверхности, $C \subset S$ - росток эффективной (возможно, приводимой) кривой, $x \in C$. Рассмотрим некоторую последовательность раздутий

$$
\varphi_{i, i-1}: S_{i} \rightarrow S_{i-1}
$$

$S_{0}=S, i=1, \ldots, N, \varphi_{i, i-1}$ раздувает точку $x_{i-1} \in S_{i-1}, E_{i}=\varphi_{i, i-1}^{-1}\left(x_{i-1}\right) \subset S_{i}$ - исключительная прямая. Положим для $i>j$

$$
\varphi_{i, j}=\varphi_{i, i+1, j} \circ \cdots \circ \varphi_{i, i-1}: S_{i} \rightarrow S_{j},
$$

$\varphi=\varphi_{N, 0}, \widetilde{S}=S_{N}$. Мы предполагаем, что раздуваемые точки $x_{i}$ лежат одна над другой, т. е. $x_{i} \in E_{i}$, и что $x_{0}=x$, так что все точки $x_{i}, i \geqslant 1$, лежат над $x$ :

$$
\varphi_{i, 0}\left(x_{i}\right)=x \in S
$$

Пусть $\Gamma$ - граф с вершинами $1, \ldots, N$ и ориентированными ребрами (стрелками) $i \rightarrow j$, которые соединяют вершины $i$ и $j$, если и только если $i>j$ и

$$
x_{i-1} \in E_{j}^{i-1},
$$

где для кривой $Y \subset S_{j}$ ее собственный прообраз на $S_{a}, a \geqslant j$, обозначаются символом $Y^{a}$.

Предположим, что точка $x$ есть изолированная не логканоническая особенность пары $\left(S, n^{-1} C\right)$ для некоторого $n \geqslant 1$. Это означает, что для некоторого исключительного дивизора $E \subset \widetilde{S}$ выполнено лог неравенство Нётера-Фано

$$
\nu_{E}(C)=\operatorname{ord}_{E} \varphi^{*} C>n\left(a_{E}+1\right)
$$

где $a_{E}$ - дискрепантность $E$. Без ограничения общности можно считать, что $E=E_{N}-$ последний исключительный дивизор.

Как обычно, пусть для $i>j$ символ $p_{i j}$ обозначает число путей в графе $\Gamma$ из вершины $i$ в вершину $j$, для $i<j$ полагаем $p_{i j}=0$, и, как всегда, $p_{i i}=1$. $\mathrm{B}$ терминах чисел $p_{i j}$ логнеравенство Нётера-Фано (52) приобретает традиционный вид

$$
\sum_{i=1}^{N} p_{N i} \mu_{i}>n\left(\sum_{i=1}^{N} p_{N i}+1\right)
$$

где $\mu_{i}=$ mult $_{x_{i-1}} C^{i-1}$.

ПреДЛОЖЕНИЕ 3.7. Имеет место следующая альтернатива: либо $\mu_{1}>2 n$ (т.е. уже первый исключительный дивизор $E_{1} \subset S_{1}$ реализует не логтерминальную особенность пары $\left.\left(S, n^{-1} C\right)\right)$, либо $N \geqslant 2$ и выполнено неравенство

$$
\mu_{1}+\mu_{2}>2 n
$$


ДоказАтельство. Если $N=1$, то $\mu_{1}>2 n$ в силу логнеравенства НётераФано. Предположим, что $\mu_{1} \leqslant 2 n$, тогда $N \geqslant 2$. Очевидно, что $\mu_{1}>n$. Если $\mu_{2} \geqslant n$, то $\mu_{1}+\mu_{2}>2 n$, как и утверждалось. Предположим, что $\mu_{2}<n$. Тогда для любого $i \in\{2, \ldots, N\}$, имеем $\mu_{i} \leqslant \mu_{2}<n$ (поскольку точка $x_{i-1}$ лежит над $\left.x_{1}\right)$. Стало быть, из неравенства (53) получаем

$$
p_{N 1}\left(\mu_{1}-n\right)+\sum_{i=2}^{N} p_{N i}\left(\mu_{2}-n\right)>n .
$$

Однако

$$
p_{N 1}=\sum_{j \rightarrow 1} p_{N j} \leqslant \sum_{i=2}^{N} p_{N i}
$$

так что тем более

$$
\sum_{i=2}^{N} p_{N i}\left(\mu_{1}+\mu_{2}-2 n\right)>n .
$$

Следовательно, $\mu_{1}+\mu_{2}>2 n$. Предложение доказано.

Завершим теперь доказательство предложения 3.6. Рассмотрим общую поверхность $S \ni x$. Пара $\left(S, D_{S}\right)$ не логканонична, но вне точки $x$ логканонична. Согласно предложению 3.7 , либо mult $_{x} D_{S}>2$, но тогда и mult ${ }_{x} D>2$, так что реализуется первая из двух возможностей предложения 3.6 , либо пара $\left(S^{+}, D_{S}^{+}\right)$ (собственный прообраз пары $\left(S, D_{S}\right)$ на $\left.X^{+}\right)$не логканонична, но логканонична вне некоторого собственного замкнутого связного подмножества

$$
Z_{S} \subset E_{S}=E \cap S^{+} \cong \mathbb{P}^{1} \text {. }
$$

Очевидно, что $Z_{S}$ есть точка $y_{S} \in E_{S}$. В силу общности поверхности $S$ существует гиперплоскость $B \subset E$ такая, что

$$
y_{S}=B \cap S^{+} .
$$

Согласно предложению 3.7 , выполнено неравенство

$$
\operatorname{mult}_{x} D_{S}+\text { mult }_{y_{S}} D_{S}^{+}>2 \text {. }
$$

Отсюда немедленно вытекает неравенство (51) и предложение 3.6.

2.3. Изолированные особенности. В качестве еще одного приложения принципа связности рассмотрим росток $x \in X$ изолированной терминальной особенности со следующими свойствами. Пусть

$$
\varphi: X^{+} \rightarrow X
$$

- раздутие точки $x, E=\varphi^{-1}(x)$ - неприводимый приведенный исключительный дивизор. Многообразия $X, X^{+}$и $E$ имеют $\mathbb{Q}$-факториальные терминальные особенности. Пусть $\delta=a(E, X)$ - дискрепантность $E, D$ - эффективный $\mathbb{Q}$-дивизор на $X, D^{+}$- его собственный прообраз на $X^{+}$. Определим число $\nu_{E}(D)$ формулой

$$
\varphi^{*} D=D^{+}+\nu_{E}(D) E
$$


ПРЕДЛОЖЕНИЕ 3.8. Предположим, что пара $(X, D)$ не канонична в точке $x$, которая является изолированным центром неканонической особенности этой пары. Предположим также, что для некоторого целого $k \geqslant 1$ выполнено неравенство

$$
\nu_{E}(D)+k \leqslant \delta
$$

Тогда пара $\left(X^{+}, D^{+}\right)$не логканонична и найдется не логканоническая особенность $\widetilde{E} \subset \widetilde{X}$ этой пары (где $\widetilde{X} \rightarrow X^{+}-$некоторая модель), иентр которой

$$
\operatorname{centre}\left(\widetilde{E}, X^{+}\right) \subset E
$$

имеет размерность $\geqslant k$.

ДокАЗАтельство. Предполагая $X \subset \mathbb{P}^{N}$ проективно вложенным, рассмотрим общее линейное подпространство $P \subset \mathbb{P}^{N}$ коразмерности $k$, содержащее точку $x$. Пусть $\Lambda_{P}$ - линейная система гиперплоскостей, содержащих $P$, и $\Lambda-$ соответствующая линейная система сечений многообразия $X$. Пусть $\varepsilon>0-$ достаточно малое рациональное число вида $1 / k$ и $\left\{H_{I} \mid i \in I\right\} \subset \Lambda$ - набор общих дивизоров в количестве $\sharp I=K k$. Положим

$$
R=D+\sum_{i \in I} \varepsilon H_{i}
$$

и пусть $R^{+}$- собственный прообраз $R$ на $X^{+}$.

Очевидно, пара $\left(X^{+}, D^{+}\right)$не логканонична. Центр любой ее не логканонической особенности содержится в $E$. Далее, нелогканоничность - открытое свойство, так что, несколько уменьшая коэффициенты в $D$, можно считать, что выполнена строгая версия неравенства $(54)$, т. е. $\nu_{E}(D)+k<\delta$ (с сохранением остальных предположений).

Рассмотрим теперь пару $\left(X^{+}, R^{+}\right)$(мы продолжаем считать $X \ni x$ ростком, так что все построения локальны в окрестности точки $x$ ). Она не логканонична, и все ее не логканонические особенности суть не логканонические особенности пары $\left(X^{+}, D^{+}\right)$, за исключением одной дополнительной особенности - ростка $(P \cap X)^{+}$сечения $X$ плоскостью $P$, т. е. базисного множества системы $\Lambda$. В силу строгого варианта неравенства $(54)$, класс $-\left(K_{X^{+}}+R^{+}\right)$очевидным образом $\varphi$-численно эффективен и $\varphi$-объемен, так что, применяя (к $X=X^{+}, Z=X$, $h=\varphi, D=R^{+}$) принцип связности, заключаем, что объединение центров не логканонических особенностей пары $\left(X^{+}, R^{+}\right)$на $X^{+}$связно. В силу общности $P$ это возможно, только если $(P \cap X)^{+}$пересекает какой-то центр не логканонической особенности пары $\left(X^{+}, D^{+}\right)$, который должен иметь размерность не меньше $k$. Этим предложение доказано.

Доказанный факт будет применяться к интересующему нас случаю гиперповерхностной особенности $x \in X$, с гладким исключительным дивизором.

2.4. Особенности пар на гладкой гиперповерхности. Пусть $X \subset \mathbb{P}^{N}-$ гладкая гиперповерхность степени $m \in\{2, \ldots, N-1\}, D \in\left|l H_{X}\right|$ - эффективный дивизор, высекаемый на $X$ гиперповерхностью степени $l \geqslant 1$ (так что $H_{X}$ - класс гиперплоского сечения $\left.X\right)$. Следующий факт и его доказательство хорошо известны [47], [48]. 
ПРЕДЛОЖЕНИЕ 3.9. Для любого $n \geqslant l$ nара $\left(X, n^{-1} D\right)$ логканонична.

ДокАЗАТЕЛЬСтво. Можно считать, что $n=l$. Предположим противное: пара $\left(X, n^{-1} D\right)$ не логканонична. Поскольку для любой кривой $C \subset X$ справедливо неравенство mult $_{C} D \leqslant n$ (см. [1; лемма 2.1]), центром не логканонической особенности пары $\left(X, n^{-1} D\right)$ может быть только точка. Пусть $x \in X$ - такая точка. Рассмотрим теперь общую проекцию $\pi: \mathbb{P}^{N} \rightarrow \mathbb{P}^{N-1}$. Ее ограничение на $X$ есть конечный морфизм $\pi_{X}: X \rightarrow \mathbb{P}^{N-1}$ степени $m$, который является аналитическим изоморфизмом в точке $x$, и можно предполагать, что

$$
\pi_{X}^{-1}\left(\pi_{X}(x)\right) \cap \operatorname{Supp} D=\{x\} .
$$

Отсюда следует, что росток пары $\left(X, n^{-1} D\right)$ в точке $x$ и росток пары $\left(\mathbb{P}^{N-1}\right.$, $\left.n^{-1} \pi(D)\right)$ в точке $\pi(x)$ аналитически изоморфны. В частности, точка $\pi(x)$ есть изолированный центр не логканонической особенности пары $\left(\mathbb{P}^{N-1}, n^{-1} \pi(D)\right)$. Однако такого не может быть.

Нелогканоничность - открытое свойство, так что для рационального числа $s<n^{-1}$, достаточно близкого к $n^{-1}$, пара $\left(\mathbb{P}^{N-1}, s \pi(D)\right)$ все еще имеет точку $\pi(x)$ изолированным центром не логканонической особенности. Пусть $P \subset$ $\mathbb{P}^{N-1}$ - гиперплоскость, не содержащая точку $\pi(x)$. Поскольку $s m n+1<N$, то $\mathbb{Q}$-дивизор $-\left(K_{\mathbb{P}^{N-1}}+s \pi(D)+P\right)$ обилен, так что можно применить к паре

$$
\left(\mathbb{P}^{N-1}, s \pi(D)+P\right)
$$

принцип связности Шокурова-Коллара (в обозначениях теоремы $3.2, X=\mathbb{P}^{N-1}$, $Z$ - точка, в качестве $\mathbb{Q}$-дивизора $D$ берется $s \pi(D)+P$, условия теоремы 3.2 выполнены тривиальным образом в силу сказанного) и получить противоречие: точка $\pi(x)$ - изолированный центр не логканонической особенности и дивизор $P$ входит в $\mathbb{Q}$-дивизор $s \pi(D)+P$ с коэффициентом единица, однако $\pi(x) \notin P$, так что связность нарушена. Предложение доказано.

2.5. Локальное неравенство для изолированной гиперповерхностной особенности. Пусть $x \in X$ - росток изолированной гиперповерхностной терминальной особенности. Точнее, если $\varphi: X^{+} \rightarrow X-$ раздутие точки $x$ и $\varphi^{-1}(x)=E \subset X^{+}$- исключительный дивизор, то предполагаем, что $X^{+}$и $E-$ гладкие, причем $E$ изоморфен гладкой гиперповерхности степени $\mu=$ mult $_{o} V$ в $\mathbb{P}^{M}$.

Далее, пусть $D \ni x$ - росток простого дивизора, $D^{+} \subset X^{+}-$его собственный прообраз, $D^{+} \sim-\nu E$ для $\nu \in \mathbb{Z}_{+}$, так что имеет место равенство

$$
\operatorname{mult}_{o} D=\mu \nu
$$

ПРЕДЛОЖЕНИЕ 3.10. Предположим, что пара $\left(X, n^{-1} D\right)$ не канонична в точке $x$, которая является изолированным центром неканонической особенности этой пары. Тогда имеет место неравенство

$$
\nu>n
$$


ДокАЗАТЕЛЬСтво. Предположим противное: $\nu \leqslant n$. Пара $\left(X^{+}, n^{-1} D^{+}\right)$тогда не канонична, причем центр любой неканонической особенности этой пары (т. е. любой максимальной особенности дивизора $D^{+}$) содержится в исключительном дивизоре $E$. В силу обращения присоединения пара $\left(E, n^{-1} D_{E}^{+}\right)$, где $D_{E}^{+}=\left.D^{+}\right|_{E}$, не логканонична. Пусть $H_{E}=-\left.E\right|_{E}-$ образующая группы Ріс $E$, т. е. гиперплоское сечение $E$ относительно вложения $E \subset \mathbb{P}^{M}$. Имеем

$$
D_{E}^{+} \sim-\left.\nu E\right|_{E}=\nu H_{E}
$$

Поскольку $\nu \leqslant n$, не логканоничность пары $\left(E, n^{-1} D_{E}^{+}\right)$противоречит предложению 3.9. Доказательство закончено.

Рассмотрим в качестве еще одного приложения принципа связности следующую локальную ситуацию. Пусть $x \in X$ - росток изолированной квадратичной особенности, $\operatorname{dim} X \geqslant 3$. Раздуем точку $x$ :

$$
\lambda: X^{+} \rightarrow X
$$

символом $E$ обозначим исключительный дивизор $\lambda^{-1}(x)$, который будем рассматривать как квадратичную гиперповерхность $E \subset \mathbb{P}^{\operatorname{dim} X}$. Пусть, далее, $D-$ эффективный $\mathbb{Q}$-Картье дивизор на многообразии $X$ и $D^{+}-$его собственный прообраз на $X^{+}$. Предполагая исключительную квадрику $E$ неприводимой, определим число $\beta \in \mathbb{Q}_{+}$соотношением

$$
D^{+} \sim \lambda^{*} D-\beta E
$$

ПРЕДЛОЖЕНИЕ 3.11. Предположим, что ранг квадратичной гиперповерхности $E$ не менъше 4, а пара $(X, D)$ имеет точку $x$ изолированным центром не канонической особенности, т.е. она не канонична, но канонична вне точки $x$. Тогда имеет место неравенство $\beta>1$.

ДокАзАТЕЛЬство. Если $\operatorname{dim} X=3$, то в силу предположения точка $x \in X$ есть невырожденная квадратичная особенность, и этот факт хорошо известен [21]. (Если $\beta \leqslant 1$, то пара $\left(X^{+}, D^{+}\right)$не канонична, так что в силу обращения присоединения пара $\left(E, D_{E}^{+}\right)$не логканонична, но $E \cong \mathbb{P}^{1} \times \mathbb{P}^{1}$ и $D_{E}^{+}$- эффективная кривая бистепени $(\beta, \beta)$, что невозможно [20].) Если $\operatorname{dim} X \geqslant 4$, то, ограничивая $D$ на общее гиперплоское сечение $Y \ni x$ многообразия $X$ относительно какого-нибудь вложения $X \hookrightarrow \mathbb{P}^{N}$ и повторяя эту процедуру $\operatorname{dim} X-3$ раз, сводим задачу (в силу обращения присоединения) к уже разобранному случаю $\operatorname{dim} X=3$. Предложение полностью доказано.

\section{§ 3. Двойные пространства индекса два}

В данном параграфе намечено доказательство теоремы 1.10 о двойных пространствах индекса два. Краткость изложения связана, во-первых, с тем, что эти многообразия не являются бирационально жесткими (в смысле любого из используемых в настоящее время определений бирациональной жесткости 
и сверхжесткости) и поэтому выходят за рамки настоящего обзора (посвященного бирационально жестким многообразиям), а во-вторых, с физическим объемом доказательства (см. [35]), так что его невозможно здесь привести. Мы лишь опишем его ключевые шаги.

В основе теоремы 1.10 лежит некоторое утверждение о виртуальном пороге канонического присоединения подвижной линейной системы на $V$. Для произвольного линейного подпространства $P \subset \mathbb{P}$ коразмерности два пусть $V_{P}-$ раздутие подмногообразия $\sigma^{-1}(P) \subset V$ (оно неприводимо, потому что многообразие $V$ предполагается общим). Для подвижной линейной системы $\Sigma$ на $V$ символом $\Sigma_{P}$ обозначим ее собственный прообраз на $V_{P}$.

Теорема 1.10 вытекает из следующего технического факта.

ТЕОРема 3.3. Предположим, что для подвижной линейной системы $\Sigma$ справедливо неравенство

$$
c_{\text {virt }}(\Sigma)<c(\Sigma, V)
$$

Тогда существует однозначно определенное линейное подпространство $P \subset \mathbb{P}$ коразмерности два, удовлетворяющее неравенству

$$
\text { mult }_{\sigma^{-1}(P)} \Sigma>c(\Sigma, V)
$$

а для собственного прообраза $\Sigma_{P}$ имеет место равенство

$$
c_{\mathrm{virt}}(\Sigma)=c_{\mathrm{virt}}\left(\Sigma_{P}\right)=c\left(\Sigma_{P}, V_{P}\right) .
$$

Выведем теорему 1.10 из теоремы 3.3. Зафиксируем подвижную линейную систему $\Sigma$, удовлетворяющую неравенству (56). Переходя, если нужно, к симметрической степени системы $\Sigma$, можно считать, что $\Sigma \subset|2 n H|=\left|-n K_{V}\right|$, где $n \geqslant 1$ - целое положительное число. Система $\Sigma$ и число $n$ фиксированы на протяжении всего доказательства. Очевидно, что $c(\Sigma, V)=n$. Согласно теореме 3.3 , существует (и единственно) линейное подпространство $P \subset \mathbb{P}$ коразмерности два, удовлетворяющее оценке $\operatorname{mult}_{R} \Sigma>n$, где $R=\sigma^{-1}(P)-$ неприводимое многообразие. Особенности многообразия $R$ для общего многообразия $V$, самое большее, нульмерны. Пусть $\varphi: V^{+} \rightarrow V-$ раздутие (возможно, особого) подмногообразия $R=\sigma^{-1}(P)$ и $E=\varphi^{-1}(R)$ - исключительный дивизор.

Лемма 3.5. (i) Многообразие $V^{+}$факториально и имеет, самое большее, конечное число изолированных двойных точек (не обязательно невырожден$H b x)$.

(ii) Линейная проекция $\pi_{\mathbb{P}}: \mathbb{P} \rightarrow \mathbb{P}^{1}$ из плоскости $P$ порождает регулярную проекиию

$$
\pi=\pi_{\mathbb{P}} \circ \sigma \circ \varphi: V^{+} \rightarrow \mathbb{P}^{1},
$$

y которой общиц слой $F_{t}=\pi^{-1}(t), t \in \mathbb{P}^{1},-$ неособое многообразие Фано индекса единица, а конечное число особых слоев имеют изолированные двойные точки.

(iii) Имеют место равенства

$$
\operatorname{Pic} V^{+}=\mathbb{Z} H \oplus \mathbb{Z} E=\mathbb{Z} K^{+} \oplus \mathbb{Z} F,
$$


где $H=\varphi^{*} H$ (для упрощения обозначений), $K^{+}=K_{V^{+}}-$канонический класс многообразия $V^{+}$и $F-$ класс слоя проекиии $\pi$, причем

$$
K^{+}=-2 H+E, \quad F=H-E .
$$

ДокАЗАТЕЛЬСтво. Эти утверждения непосредственно вытекают из определения раздутия $\varphi$, общности многообразия $V$ и следующего хорошо известного факта: изолированная гиперповерхностная особенность многообразия размерности $\geqslant 4$ факториальна (см. [49], [22]).

Пусть $\Sigma^{+}$- собственный прообраз системы $\Sigma$ на раздутии $V^{+}$подмногообразия $R$. Согласно теореме 3.3 , имеет место равенство

$$
c_{\mathrm{virt}}\left(\Sigma^{+}\right)=c\left(\Sigma^{+}, V^{+}\right) .
$$

Отсюда сразу получаем следующее утверждение.

ПредлОЖЕНИЕ 3.12. Предположим, что $c_{\mathrm{virt}}\left(\Sigma^{+}\right)=0$. Тогда система $\Sigma^{+}$ составлена из пучка $|H-R|$, m.е. $\Sigma^{+} \subset|2 n F|$.

ДокАзАТЕльство. Предположим противное:

$$
\Sigma^{+} \subset\left|-m K^{+}+l F\right|,
$$

где $m \geqslant 1$. В силу части (iii) леммы 3.5 ,

$$
m=2 n-\nu, \quad l=2 \nu-2 n \geqslant 2,
$$

так что для порога канонического присоединения имеем $c\left(\Sigma^{+}, V^{+}\right)=m$. Поскольку $c_{\text {virt }}\left(\Sigma^{+}\right)=0$, в силу теоремы 3.3 получаем $m=0$, что и утверждалось. Предложение доказано.

Вернемся к доказательству теоремы 1.10. В качестве линейной системы $\Sigma$ возьмем собственный прообраз относительно $\chi$ любой линейной системы вида $\lambda^{*} \Lambda$, где $\Lambda$ - подвижная система на базе $S$. Применение теоремы 3.3 и предложения 3.12 завершает доказательство.

Из приведенных рассуждений видно, что в основе доказательства теоремы 1.10 лежат два ключевых утверждения:

1) существование (и единственность) максимального подмногообразия вида $\sigma^{-1}(P)$, где $P \subset \mathbb{P}$ - линейное подпространство коразмерности два и

2) равенство (57) порогов канонического присоединения.

В доказательстве утверждения 1) существенную роль играет следующий локальный факт, известный как $8 n^{2}$-неравенство.

Пусть $o \in X-$ росток гладкого многообразия размерности $\operatorname{dim} X \geqslant 4$. Пусть $\Sigma$ - подвижная линейная система на $X$, а эффективный цикл $Z=\left(D_{1} \circ D_{2}\right)$, где $D_{1}, D_{2} \in \Sigma$ - общие дивизоры, - ее самопересечение. Раздуем точку о:

$$
\varphi: X^{+} \rightarrow X
$$

$E=\varphi^{-1}(o) \cong \mathbb{P}^{\operatorname{dim} X-1}-$ исключительный дивизор. Собственный прообраз системы $\Sigma$ и цикла $Z$ на $X^{+}$обозначаем символами $\Sigma^{+}$и $Z^{+}$соответственно. 
ПРЕДЛОЖЕНИЕ 3.13 ( $8 n^{2}$-неравенство). Предположим, что пара $\left(X, n^{-1} \Sigma\right)$ не канонична, но канонична вне точки о, где $n$ - некоторое положительное число. Тогда существует линейное подпространство $P \subset E$ коразмерности два (относительно E) такое, что выполнено неравенство

$$
\operatorname{mult}_{o} Z+\operatorname{mult}_{P} Z^{+}>8 n^{2} .
$$

Эквивалентное, но громоздко формулируемое утверждение неоднократно публиковалось И. А. Чельцовым [50]-[52], однако в его рассуждениях имеется существенный пробел (см. [53]). Полное доказательство см. в [53].

Наметим теперь доказательство равенства (57), т. е. утверждения 2).

Предположим, что справедливо неравенство

$$
c_{\mathrm{virt}}\left(\Sigma^{+}\right)<c\left(\Sigma^{+}, V^{+}\right)=m .
$$

Тогда пара $\left(V^{+}, m^{-1} \Sigma^{+}\right)$не канонична, так что линейная система $\Sigma^{+}$обладает максимальной особенностью, т. е. для некоторого бирационального морфизма $\psi: \widetilde{V} \rightarrow V^{+}$и неприводимого исключительного дивизора $E^{+} \subset \widetilde{V}$ выполнено неравенство Нётера-Фано:

$$
\nu_{E}\left(\Sigma^{+}\right)>m a\left(E^{+}, V^{+}\right) .
$$

Лемма 3.6. Центр максималъной особенности $E^{+}$содержится в некотором слое $F_{t}=\pi^{-1}(t)$, m.е. $B=\pi \circ \psi\left(E^{+}\right)=t \in \mathbb{P}^{1}$.

ДокАЗАТЕЛЬСтво. Предположим противное: $\pi \circ \psi\left(E^{+}\right)=\mathbb{P}^{1}$. Ограничивая линейную систему $\Sigma^{+}$на слой общего положения $F=F_{s}$, получим, что пара

$$
\left(F, m^{-1} \Sigma_{F}\right)
$$

где $\Sigma_{F} \subset\left|-m K_{F}\right|$, не канонична. Однако $F$ - гладкое двойное пространство индекса 1 и хорошо известно [54], что это невозможно. Лемма доказана.

Для упрощения обозначений пусть $F=F_{t}$ - слой, содержащий центр особенности $E^{+}$.

ПРЕДЛОЖЕНИЕ 3.14. Центр В есть особая точка слоя $F$ и многообразия $V$.

Доказательство см. в [35].

Пусть теперь $\lambda: V^{\sharp} \rightarrow V^{+}-$раздутие точки $o, E^{\sharp}=\lambda^{-1}(o) \subset V^{\sharp}-$ исключительный дивизор, который можно рассматривать как квадратичную гиперповерхность в $\mathbb{P}^{M}$. Можно показать [35], что при $M \geqslant 6$ для общей гиперповерхности $W \subset \mathbb{P}$, произвольной плоскости $P \subset \mathbb{P}$ коразмерности два и любой особенности $o \in V^{+}$ранг квадрики $E^{\sharp}$ не меньше 4 . Определим число $\beta \in \mathbb{Z}_{+}$ соотношением

$$
D^{\sharp} \sim \lambda^{*} D-\beta E^{\sharp},
$$

где $D \in \Sigma^{+}-$общий дивизор, $D^{\sharp}$ - его собственный прообраз на $V^{\sharp}$. К общему многообразию $V$ применимо предложение 3.11 , которое дает неравенство

$$
\beta>m \text {. }
$$


Далее, дивизор $\lambda_{F}^{*} D_{F}-\beta E_{F}^{\sharp}$ на собственном прообразе $F^{\sharp} \subset V^{\sharp}$ эффективен (символы $\lambda_{F}$ и $E_{F}^{\sharp}$ означают раздутие точки $o \in F$ и исключительный дивизор $\lambda_{F}^{-1}(o)$ соответственно). Отсюда следует, что выполнено неравенство

$$
\operatorname{mult}_{o} D_{F} \geqslant 2 \beta>2 m,
$$

что невозможно ( $H$-степень дивизора $D_{F}$ как эффективного алгебраического цикла есть $2 m)$. Этим совпадение порогов (57) для $M \geqslant 6$ доказано.

\section{Список литературы}

[1] А. В. Пухликов, "Бирационально жесткие многообразия. І. Многообразия Фано", УМH, 62:5 (2007), 15-106; англ. пер.: А. V. Pukhlikov, "Birationally rigid varieties. I. Fano varieties", Russian Math. Surveys, 62:5 (2007), 857-942.

[2] В. Г. Саркисов, "О структурах расслоений на коники", Изв. АН СССР. Сер. матем., 46:2 (1982), 371-408; англ. пер.: V. G. Sarkisov, "On conic bundle structures", Math. USSR-Izv., 20:2 (1983), 355-390.

[3] А.В. Пухликов, "Бирациональные автоморфизмы трехмерных алгебраических многообразий с пучком поверхностей дель Пеццо", Изв. РАН. Сер. матем., 62:1 (1998), 123-164; англ. пер.: A. V. Pukhlikov, "Birational automorphisms of algebraic threefolds with a pencil of Del Pezzo surfaces", Izv. Math., 62:1 (1998), 115-155.

[4] М. М. Гриненко, "Расслоения на поверхности дель Пеццо", УМН, 61:2 (2006), 67-112; англ. пер.: M. M. Grinenko, "Fibrations into del Pezzo surfaces", Russian Math. Surveys, 61:2 (2006), 255-300.

[5] Ю. И. Манин, Кубические формы. Алгебра, геометрия, арифметика, Наука, М., 1972, 304 с.; англ. пер.: Yu. I. Manin, Cubic forms. Algebra, geometry, arithmetic, 2nd ed., North-Holland Math. Library, 4, North-Holland, Amsterdam-New YorkOxford, 1986.

[6] А.В. Пухликов, "Послойные бирациональные соответствия", Матем. заметки, 68:1 (2000), 120-130; англ. пер.: A. V. Pukhlikov, "Fiberwise birational correspondences", Math. Notes, 68:1-2 (2000), 102-112.

[7] А.В. Пухликов, "Бирационально жесткие многообразия с пучком двойных накрытий Фано. III", Матем. сб., 197:3 (2006), 35-68; англ. пер.: А. V. Pukhlikov, "Birationally rigid varieties with a pencil of Fano double covers. III", Sb. Math., 197:3 (2006), 335-368.

[8] A. V. Pukhlikov, "Birational geometry of algebraic varieties with a pencil of Fano cyclic covers", Pure Appl. Math. Q., 5:2 (2009), 641-700.

[9] И. Р. Шафаревич, Б. Г. Авербух, Ю. Р. Вайнберг, А. Б. Жижченко, Ю. И. Манин, Б. Г. Мойшезон, Г. Н. Тюрина, А. Н. Тюрин, "Алгебраические поверхности”, Тр. МИАН, 75, Наука, М., 1965, 3-215.

[10] C. Birkar, P. Cascini, C. D. Hacon, J. McKernan, "Existence of minimal models for varieties of log general type", J. Amer. Math. Soc., 23:2 (2010), 405-468.

[11] C. D. Hacon, J. McKernan, The Sarkisov program, arXiv: abs/0905.0946.

[12] A. Corti, "Factoring birational maps of threefolds after Sarkisov", J. Algebraic Geom., 4:2 (1995), 223-254.

[13] V. G. Sarkisov, Birational maps of standard $\mathbb{Q}$-Fano fibrations, Preprint, Kurchatov Institute of Atomic Energy, Moscow, 1989.

[14] M. Reid, Birational geometry of 3-folds according to Sarkisov, Preprint, Warwick Univ., 1991. 
[15] В. А. Исковских, "Бирациональная жесткость гиперповерхностей Фано в рамках теории Мори", УМН, 56:2 (2001), 3-86; англ. пер.: V. A. Iskovskikh, "Birational rigidity of Fano hypersurfaces in the framework of Mori theory", Russian Math. Surveys, 56:2 (2001), 207-291.

[16] V. Alexeev, "Two two-dimensional terminations", Duke Math. J., 69:3 (1993), $527-545$.

[17] I. Cheltsov, J. Park, J. Won, Log canonical thresholds of certain Fano hypersurfaces, arXiv: abs/0706.0751.

[18] C. Birkar, "Ascending chain condition for log canonical thresholds and termination of log flips", Duke Math. J., 136:1 (2007), 173-180.

[19] В. А. Исковских, Ю.И. Манин, "Трехмерные квартики и контрпримеры к проблеме Люрота", Матем. сб., 8:1(9) (1971), 140-166; англ. пер.: V. A. Iskovskih, J.I. Manin, "Three-dimensional quartics and counterexamples to the Lüroth problem", Math. USSR-Sb., 15:1 (1971), 141-166.

[20] А.В. Пухликов, "Бирациональные автоморфизмы трехмерной квартики с простейшей особенностью", Матем. сб., 135:4 (1988), 472-496; англ. пер.: А. V. Pukhlikov, "Birational automorphisms of a three-dimensional quartic with a quadratic singularity", Math. USSR-Sb., 63:2 (1989), 457-482.

[21] A. Corti, "Singularities of linear systems and 3-fold birational geometry", Explicit birational geometry of 3-folds, London Math. Soc. Lecture Note Ser., 281, Cambridge Univ. Press, Cambridge, 2000, 259-312.

[22] A. Corti, A. Pukhlikov, M. Reid, "Fano 3-fold hypersurfaces", Explicit birational geometry of 3-folds, London Math. Soc. Lecture Note Ser., 281, Cambridge Univ. Press, Cambridge, 2000, 175-258.

[23] A. Corti, M. Mella, "Birational geometry of terminal quartic 3-folds. I", Amer. J. Math., 126:4 (2004), 739-761.

[24] M. Mella, "Birational geometry of quartic 3-folds. II. The importance of being $\mathbb{Q}$-factorial", Math. Ann., 330:1 (2004), 107-126.

[25] G. Brown, A. Corti, F. Zucconi, "Birational geometry of 3-fold Mori fiber spaces", The Fano Conference, Univ. Torino, Turin, 2004, 235-275.

[26] М. М. Гриненко, "Бирациональные свойства пучков поверхностей дель Пеццо степеней 1 и 2", Матем. сб., 191:5 (2000), 17-38; англ. пер.: М. M. Grinenko, "Birational properties of pencils of del Pezzo surfaces of degrees 1 and 2", Sb. Math., 191:5 (2000), 633-653.

[27] М.М.Гриненко, "Бирациональные свойства пучков поверхностей дель Пеццо степеней 1 и 2. II", Матем. сб., 194:5 (2003), 31-60; англ. пер.: М. M. Grinenko, "Birational properties of pencils of del Pezzo surfaces of degrees 1 and 2. II", Sb. Math., 194:5 (2003), 669-695.

[28] И.В. Соболев, "Бирациональные автоморфизмы одного класса многообразий, расслоенных на кубические поверхности", Изв. РАН. Сер. матем., 66:1 (2002), 203-224; англ. пер.: I. V. Sobolev, "Birational automorphisms of a class of varieties fibred into cubic surfaces", Izv. Math., 66:1 (2002), 201-222.

[29] М. М. Гриненко, "Структуры Мори на трехмерном многообразии Фано индекса 2 и степени 1", Алгебраическая геометрия: Методы, связи и приложения, Тр. МИАН, 246, Наука, М., 2004, 116-141; англ. пер.: M. M. Grinenko, "Mori structures on a Fano threefold of index 2 and degree 1", Proc. Steklov Inst. Math., 246:3 (2004), 103-128.

[30] В.А. Исковских, "О проблеме рациональности для трехмерных алгебраических многообразий, расслоенных на поверхности Дель Пеццо", Тр. МИАН, 208, Наука, М., 1995, 128-138; англ. пер.: V. A. Iskovskikh, "On the rationality problem for three-dimensional algebraic varieties fibered over del Pezzo surfaces", Proc. Steklov Inst. Math., 208 (1995), 115-123. 
[31] А.В. Пухликов, "Бирационально жесткие многообразия с пучком двойных накрытий Фано. II", Матем. сб., 195:11 (2004), 119-156; англ. пер.: А. V. Pukhlikov, "Birationally rigid varieties with a pencil of Fano double covers. II", Sb. Math., 195:11 (2004), 1665-1702.

[32] A. V. Pukhlikov, "Birational geometry of algebraic varieties with a pencil of Fano complete intersections", Manuscripta Math., 121:4 (2006), 491-526.

[33] А. В. Пухликов, "Бирациональная геометрия прямых произведений Фано", Изв. РАН. Сер. матем., 69:6 (2005), 153-186; англ. пер.: A. V. Pukhlikov, "Birational geometry of Fano direct products", Izv. Math., 69:6 (2005), 1225-1255.

[34] А. В. Пухликов, "Бирациональная геометрия двойных накрытий Фано", Матем. сб., 199:8 (2008), 123-148; англ. пер.: A. V. Pukhlikov, "Birational geometry of Fano double covers", Sb. Math., 199:8 (2008), 1225-1250.

[35] A. V. Pukhlikov, Birational geometry of Fano double spaces of index two, arXiv: math.AG/0812.3863.

[36] А. В. Пухликов, "Бирационально жесткие расслоения Фано", Изв. РАН. Сер. матем., 64:3 (2000), 131-150; англ. пер.: A. V. Pukhlikov, "Birationally rigid Fano fibrations", Izv. Math., 64:3 (2000), 563-581.

[37] И.В. Соболев, "Об одной серии бирационально жестких многообразий с пучком гиперповерхностей Фано", Матем. сб., 192:10 (2001), 123-130; англ. пер.: I. V. Sobolev, "On a series of birationally rigid varieties with a pencil of Fano hypersurfaces", Sb. Math., 192:9-10 (2001), 1543-1551.

[38] А. В. Пухликов, "Бирациональные автоморфизмы алгебраических многообразий с пучком двойных квадрик", Матем. заметки, 67:2 (2000), 241-249; англ. пер.: A. V. Pukhlikov, "Birational automorphisms of algebraic varieties with a pencil of double quadrics", Math. Notes, 67:2 (2001), 192-199.

[39] Yu. I. Manin, "Rational surfaces over perfect fields", Inst. Hautes Études Sci. Publ. Math., 30 (1966), 55-113.

[40] В.В. Шокуров, "Трехмерные логперестройки”, Изв. РАН. Сер. матем., 56:1 (1992), 105-203; англ. пер.: V. V. Shokurov, "3-fold log flips", Russian Acad. Sci. Izv. Math., 40:1 (1993), 95-202.

[41] Flips and abundance for algebraic threefolds, Papers from the Second Summer Seminar on Algebraic Geometry (University of Utah, Salt Lake City, 1991), Astérisque, 211, Soc. Math. France, Paris, 1992.

[42] I. A. Cheltsov, "Fano varieties with many selfmaps", Adv. Math., 217:1 (2008), 97-124.

[43] А. В. Пухликов, "Бирациональная геометрия особых многообразий Фано", Многомерная алгебраическая геометрия, Тр. МИАН, 264, МАИК, М., 2009, 165-183; A. V. Pukhlikov, "Birational geometry of singular Fano varieties", Proc. Steklov Inst. Math., 264 (2009), 159-177.

[44] W. Fulton, Intersection Theory, Springer-Verlag, 1984; рус. пер.: У. Фултон, Теория пересечений, Мир, М., 1989.

[45] A. V. Pukhlikov, "Birational automorphisms of Fano hypersurfaces", Invent. Math., 134:2 (1998), 401-426.

[46] А. В. Пухликов, "Бирационально жесткие гиперповерхности Фано", Изв. РАН. Сер. матем., 66:6 (2002), 159-186; англ. пер.: A. V. Pukhlikov, "Birationally rigid Fano hypersurfaces", Izv. Math., 66:6 (2002), 1243-1269.

[47] И.А. Чельцов, "Лог-канонические пороги на гиперповерхностях", Матем. сб., 192:8 (2001), 155-172; англ. пер.: I. A. Cheltsov, "Log canonical thresholds on hypersurfaces", Sb. Math., 192:8 (2001), 1241-1257.

[48] А.В. Пухликов, "Бирационально жесткие гиперповерхности Фано с изолированными особенностями", Матем. сб., 193:3 (2002), 135-160; англ. пер.: 
A. V. Pukhlikov, "Birationally rigid Fano hypersurfaces with isolated singularities", Sb. Math., 193:3 (2002), 445-471.

[49] F. Call, G. Lyubeznik, "A simple proof of Grothendieck's theorem on the parafactoriality of local rings", Commutative algebra: syzygies, multiplicities, and birational algebra (South Hadley, MA, 1992), Contemp. Math., 159, Amer. Math. Soc., Providence, RI, 1994, 15-18.

[50] И. А. Чельцов, "Локальные неравенства и бирациональная сверхжесткость многообразий Фано”, Изв. РАН. Сер. матем., 70:3 (2006), 185-221; англ. пер.: I. A. Cheltsov, "Local inequalities and birational superrigidity of Fano varieties", Izv. Math., 70:3 (2006), 605-639.

[51] И. А. Чельцов, "Нерациональность четырехмерного гладкого полного пересечения квадрики и квартики, не содержащего плоскости", Матем. сб., 194:11 (2003), 95-116; англ. пер.: I. A. Cheltsov, "Non-rationality of the 4-dimensional smooth complete intersection of a quadric and a quartic not containing planes", Sb. Math., 194:11 (2003), 1679-1699.

[52] I. Cheltsov, "Double cubics and double quartics", Math. Z., 253:1 (2006), 75-86.

[53] A. V. Pukhlikov, On the $8 n^{2}$-inequality, arXiv: abs/0811.0183.

[54] А. В. Пухликов, "Бирациональные автоморфизмы двойного пространства и двойной квадрики", Изв. АН СССР. Сер. матем., 52:1 (1988), 229-239; англ. пер.: A. V. Pukhlikov, "Birational automorphisms of a double space and double quadric", Math. USSR-Izv., 32:1 (1989), 233-243.

\section{А. В. Пухликов (А. V. Pukhlikov)}

Математический институт им. В. А. Стеклова РАН;

The University of Liverpool, UK

E-mail: pukh@liv.ac.uk, pukh@mi.ras.ru
Поступила в редакцию 17.12.2009 